UNITED STATES

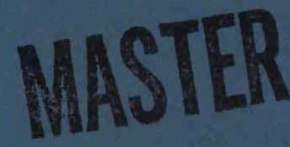

DEPARTMENT OF THE INTERIOR

GEOLOGICAL SURVEY

Federal Center, Denver, Colorado 80225

CHEMICAL AND RADIOCHEMICAL DATA FOR DOMESTIC

SUPPLY WELIS AND SURFACE-WATER SAMPLES IN THE VICINITY OF TATUM SALT DOME, LAMAR COUNTY, MISSISSIPPI

(Dribble-53)

Date Written: 1966

Date Released: 1972

Prepared Under

Agreement No. AT(29-2)-474

for the

Nevada Operations Office

U.S. Atomic Energy Commission 
This report was prepared as an account of work

sponsored by the United States Government.

Neither the United States nor the United State:

Atomic Fnergy Commission, nor any of their

employees, nor any of their contractors, sub-

contractors, or their emplayees, makes any

warranty, express or implied, or assumes an

lecal liability or respongthilfty tor the

accuracy, completeness or usefulness of any

information, apparatus, product or process

disclosed, or represents that its use would

not infringe privately owned rights.

Available from

Department of Commerce, National

Technical Information Service,

Springfield, Virginia 22151

Price: Printed copy $\$ 3.00$; Microfiche $\$ 0.95$ 


\section{DISCLAIMER}

This report was prepared as an account of work sponsored by an agency of the United States Government. Neither the United States Government nor any agency Thereof, nor any of their employees, makes any warranty, express or implied, or assumes any legal liability or responsibility for the accuracy, completeness, or usefulness of any information, apparatus, product, or process disclosed, or represents that its use would not infringe privately owned rights. Reference herein to any specific commercial product, process, or service by trade name, trademark, manufacturer, or otherwise does not necessarily constitute or imply its endorsement, recommendation, or favoring by the United States Government or any agency thereof. The views and opinions of authors expressed herein do not necessarily state or reflect those of the United States Government or any agency thereof. 


\section{DISCLAIMER}

Portions of this document may be illegible in electronic image products. Images are produced from the best available original document. 


\author{
UNITED STATES \\ DEPARTMENT OF THE INTERIOR \\ GEOLOGICAL SURVEY
}

Federa1 Center, Denver, Colorado 80225

\title{
CHEMICAL AND RADIOCHEMICAL DATA FOR DOMESTIC SUPPLY WELLS AND SURFACE-WATER SAMPLES IN THE VICINITY OF TATUM SALT DOME, LAMAR COUNTY, MISSISSIPPI
}

By

W. A. Beetem and D. B. Grove

NOTICE

This report was prepared as an account of work sponsored by the United States Government. Neither the United States nor the United States Atomic Energy Commission, nor any of their employees, nor any of their contractors, subcontractors, or their employees, makes any warranty, express or implied, or assumes any legal liability or responsibility for the accuracy, completeness or usefulness of any information, apparatus, product or process disclosed, or represents that its use would not infringe privately owned rights. 
Abstract ......................... 1

Introduction ......................... 1

We11 numbers and locations .............. 2

Summary of wells sampled............... . 8

Water quality .............. 8

Radiochemical analyses . . . . . . . . . . . 9

Chemical analyses ................ 10

References cited .................. 13

Analytical sheets .................. . 14

Chemical analyses for streams . . . . . . . . . 14

Chemical and radiochemical analyses for wells . . . . . 17

\section{ILLUSTRATIONS}

Figure 1. Map showing water-well locations within 5-mile radius of Tatum dome, Lamar County, Mississippi . . . . . . . . . . . . 3

2. Map of the Tatum salt dome area ..... . . . . 4

\section{TABLES}

Table 1. Record of Lamar County, Mississippi, wells for which chemical analyses are included ........ 5 
Dribble -53

UNITED STATES

DEPARTMENT OF THE INTERIOR

GEOLOGICAL SURVEY

USGS -474-120

Federal Center, Denver, Colorado 80225

CHEMICAL AND RADIOCHEMICAL DATA FOR DOMESTIC SUPPLY WELLS AND SURFACE-WATER SAMPLES IN THE

VICINITY OF TATUM SALT DOME,

LAMAR COUNTY, MISSISSIPPI

By

W. A. Beetem and D. B. Grove

ABSTRACT

Ground-water and surface-water samples collected in the vicinity of Tatum salt dome, Mississippi, a U.S. Atomic Energy Commission project test site, have been analyzed by the U.S. Geological Survey as part of the evaluation program after the Salmon Event. Samples were collected from wells of all types of construction and at distances of 1 to 5 miles from the dome.

\section{INTRODUCTION}

Since the Salmon Event a series of ground-water samples has been collected in the immediate vicinity (less than 1-mile distance) of the detonation. The samples were collected mainly for radiochemical analysis although chemical analyses were run on some of the samples. The radiochemical analyses have been reported in U.S. Geological Survey reports Beetem and Grove (1971a and 1971b), and Janzer, Robinson, and Rucker (1971a and 1971b). The previously unreported chemical analyses for the area studied are presented in the current report although most of the wells from which the samples were collected have not been used as domestic supplies. HT-2a and HT-2c are the only hydrologic test wells that have been used for domestic supply. Others may have been used for industrial water. 
Streams in the area have been sampled periodically. Previously unreported chemical analyses for these streams are included to make the analyses available to other users.

Brand names given in this report are included to identify specific equipment used, and do not constitute an endorsement of these products by the U.S. Geological Survey.

\section{WEILL NUMBERS AND LOCATIONS}

Eigures 1 and 2 give the location of the water wells and streamsampling locations for which chemical data are tabulated. Table 1 summarizes some of the well data for these same wells. The we11numbering system is adapted from the one used in ground-water studies in other parts of the state. Each township or part of a township within a county unit is assigned a grid letter (see figure 1), beginning with the letter A in the northwestern part of the county and continued alphabet ically in normal reading order to the southern border. The letter "I" is not used. The section number follows the grid letter. Wells in each section are numbered consecutively beginning with one. Thus, we11 number F25-2 represents we11 2 of section 25 in grid $F$. In the immediate vicinity of Tatum salt dome this system of numbering was not followed and these wells are identified as "HT" for hydrologic test well with a number following as identifier, or as " $E$ " for exploratory followed by a number. 


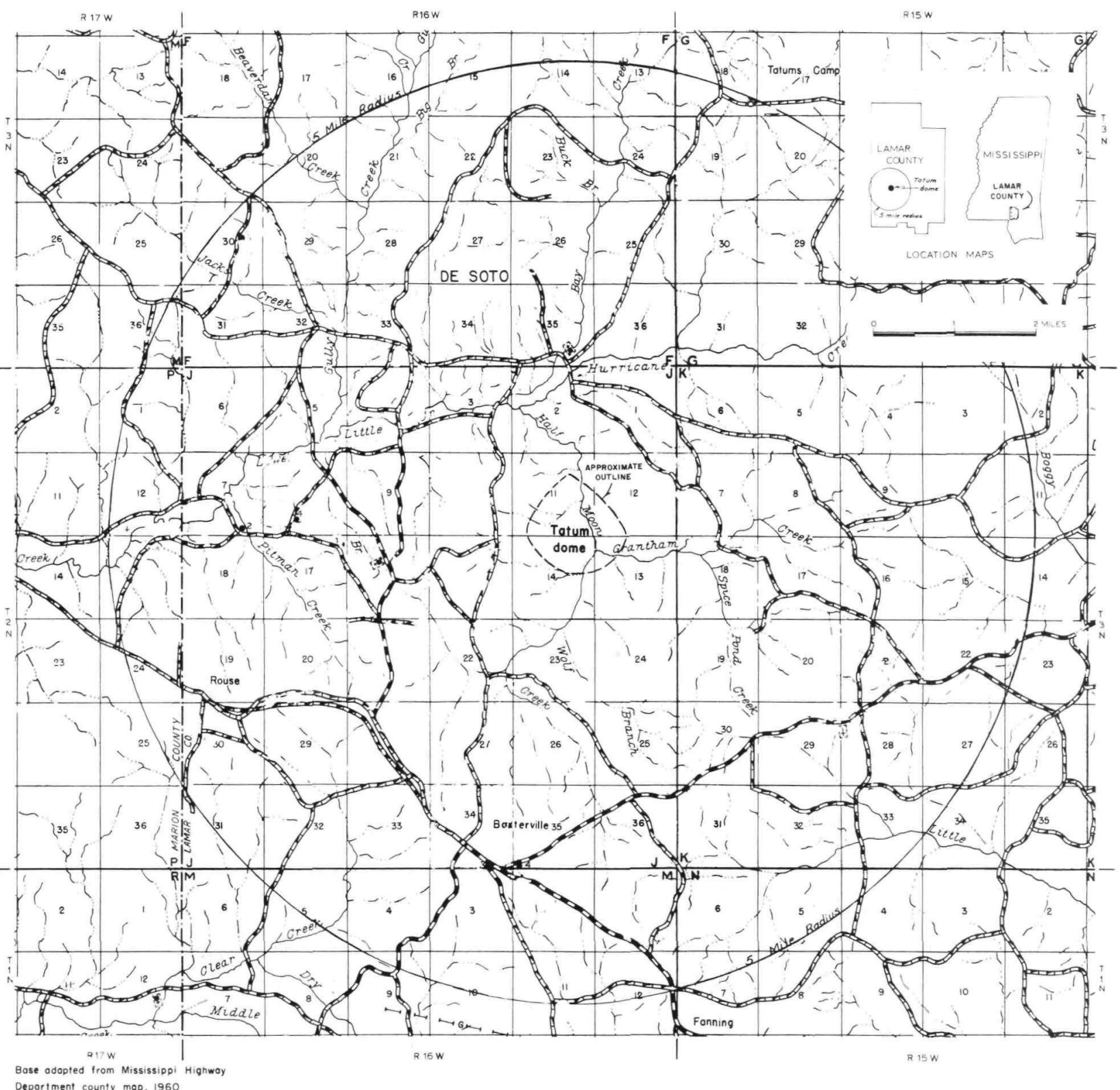

FIGURE I.--WATER-WELL LOCATIONS WITHIN 5-MILE RADIUS OF TATUM DOME, LAMAR COUNTY MISSISSIPPI. 


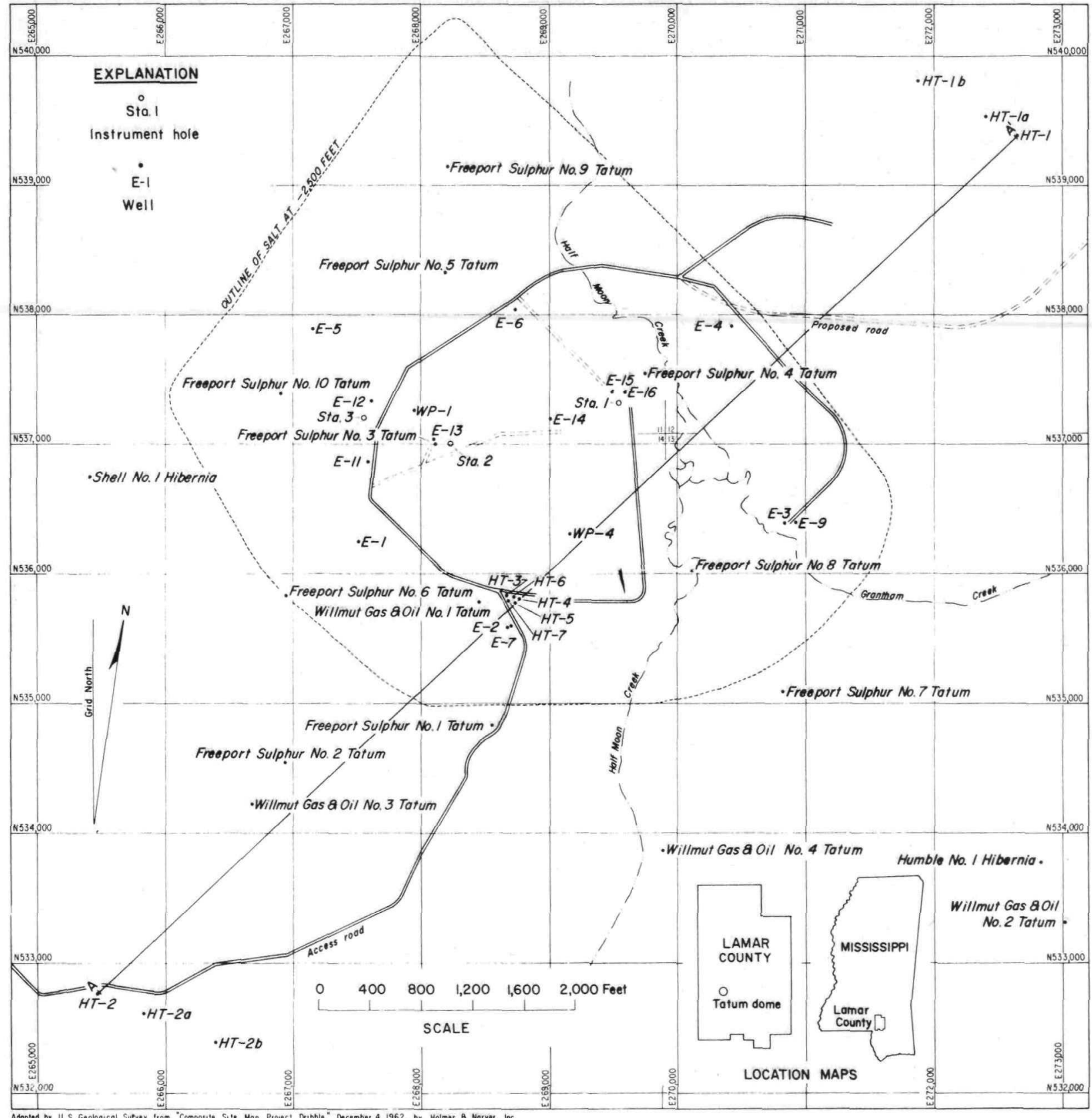

Figure I. - MAP OF TATUM DOME AREA 
Table 1.--Record of Lamar County, Mississippi, wells for which chemical analyses are included

Type of wel1: D, drilled; B, bored.

Water-bearing unit: Qt, Quaternary alluvium and terrace deposits; Tph, Tertiary Pascagoula and Hattiesburg undifferentiated; Tc, Catahoula Sandstone; Tcc, Tertiary calcite caprock; Tv, Vicksburg Group; Tcm, Cook Mountain Limestone.
Water leve1: Levels shown in feet are reported; those in tenths were measured on the dates shown. LSD: land surface datum.

Use of water: D, domestic; Ind, industrial; 0 , observation we11; PS, public supply;

S, stock.

\begin{tabular}{|c|c|c|c|c|c|c|c|c|}
\hline \multirow{2}{*}{$\begin{array}{l}\text { We11 } \\
\text { number }\end{array}$} & \multirow[b]{2}{*}{ Owner } & \multirow{2}{*}{$\begin{array}{l}\text { Year } \\
\text { drilled }\end{array}$} & \multirow{2}{*}{$\begin{array}{l}\text { Type } \\
\text { of } \\
\text { wel1 }\end{array}$} & \multirow{2}{*}{$\begin{array}{l}\text { Depth } \\
\text { (ft) }\end{array}$} & \multirow{2}{*}{$\begin{array}{c}\text { Water- } \\
\text { bearing } \\
\text { unit }\end{array}$} & \multicolumn{2}{|c|}{ Water level } & \multirow{2}{*}{$\begin{array}{l}\text { Use } \\
\text { of } \\
\text { water }\end{array}$} \\
\hline & & & & & & $\begin{array}{c}\text { Below } \\
\text { LSD }\end{array}$ & $\begin{array}{c}\text { Date of } \\
\text { measurement }\end{array}$ & \\
\hline F $25-2$ & Buel Bryant & 1945 & B & 80 & Qt & -- & -- & $\mathrm{D}, \mathrm{S}$ \\
\hline F $33-2$ & Sara Entrekin & 1951 & $\mathrm{D}$ & 230 & $\mathrm{Tph}$ & 80 & 1951 & $\mathrm{D}, \mathrm{S}$ \\
\hline F $36-5$ & J. C. Gipson & 1949 & B & 75 & Qt & 67 & -- & $\mathrm{D}, \mathrm{S}$ \\
\hline J $1-1$ & H. W. Burge & -- & $\mathrm{D}$ & 120 & Qt & 99 & 1961 & $\mathrm{D}, \mathrm{S}$ \\
\hline J $\quad 3-2$ & W. E. Brown & 1955 & B & 30 & $Q t$ & 2 & 1961 & $D, S$ \\
\hline J $\quad 4-5$ & H. 0. Thompson & 1954 & B & 31 & Qt & 16 & 1954 & $\mathrm{D}, \mathrm{S}$ \\
\hline J $\quad 4-8$ & B. F. Sones & 1959 & D & 196 & $\mathrm{Tph}$ & 60 & 1959 & $\mathrm{D}, \mathrm{S}$ \\
\hline J $8-4$ & J. R. Boler & 1959 & $\mathrm{D}$ & 280 & $\mathrm{Tph}$ & 70 & 1959 & $\mathrm{D}, \mathrm{S}$ \\
\hline J $10-1$ & Toxie Speights & -- & B & 43 & Qt & -- & -- & $\mathrm{D}, \mathrm{S}$ \\
\hline J $12-1$ & Fred Dobson & 1943 & B & 48 & Qt & 31.2 & $7-22-65$ & $\mathrm{D}, \mathrm{S}$ \\
\hline J $12-2$ & Ross Powe11 & 1951 & $\mathrm{D}$ & 90 & Qt & $-\infty$ & -- & $\mathrm{D}, \mathrm{S}$ \\
\hline J $16-7$ & Wylie Smith & 1949 & B & 66 & $Q t$ & 48.6 & $7-22-65$ & $\mathrm{D}, \mathrm{S}$ \\
\hline J $16-10$ & Archie Sistrunk & 1951 & B & 39 & Qt & 15 & 1951 & $\mathrm{D}, \mathrm{S}$ \\
\hline J $16-11$ & Mark Howe & 1952 & $\mathrm{D}$ & 100 & $Q t$ & 80 & 1952 & $D, S$ \\
\hline J $22-3$ & G. C. Saul & 1957 & D & 50 & $Q t$ & 40 & 1961. & $D, S$ \\
\hline
\end{tabular}


Table 1.--Record of Lamar County, Mississippi, wells for which chemical analyses are included--Continued

\begin{tabular}{|c|c|c|c|c|c|c|c|c|}
\hline \multirow{2}{*}{$\begin{array}{c}\text { Wel1 } \\
\text { number }\end{array}$} & \multirow[b]{2}{*}{ Owner } & \multirow{2}{*}{$\begin{array}{c}\text { Year } \\
\text { drilled }\end{array}$} & \multirow{2}{*}{$\begin{array}{c}\text { Type } \\
\text { of } \\
\text { we } 11 \\
\end{array}$} & \multirow{2}{*}{$\begin{array}{l}\text { Depth } \\
(\mathrm{ft})\end{array}$} & \multirow{2}{*}{$\begin{array}{c}\text { Water } \\
\text { bearing } \\
\text { unit }\end{array}$} & \multicolumn{2}{|c|}{ Water 1evel } & \multirow{2}{*}{$\begin{array}{c}\text { Use } \\
\text { of } \\
\text { water }\end{array}$} \\
\hline & & & & & & $\begin{array}{c}\text { Below } \\
\text { ISD }\end{array}$ & $\begin{array}{c}\text { Date of } \\
\text { measurement }\end{array}$ & \\
\hline J $25-4$ & Vol1ie DeBrow & 1956 & B & 65 & Qt & 37.4 & $4-23-65$ & $\mathrm{D}, \mathrm{S}$ \\
\hline J $25-5$ & T. C. Brashears & 1959 & D & 71 & Qt & - & $-\infty$ & $\mathrm{D}, \mathrm{S}$ \\
\hline J $26-2$ & L. C. Howard & 1958 & $\mathrm{D}$ & 80 & Qt & 60 & 1958 & $\mathrm{D}, \mathrm{S}$ \\
\hline J $27-1$ & Hubert Powe11 & 1949 & $\mathrm{D}$ & 39 & Qt & 38.6 & $4-23-65$ & $\mathrm{D}, \mathrm{S}$ \\
\hline J $32-1$ & J. Q. Butler & 1956 & $\mathrm{D}$ & 454 & $\mathrm{Tph}$ & -- & -- & $\mathrm{D}, \mathrm{S}$ \\
\hline J $34-2$ & Kerry Kittrel1 & 1960 & $\mathrm{D}$ & 132 & Qt & 68.7 & $8-14-61$ & $\mathrm{D}, \mathrm{S}$, Ind \\
\hline J $34-9$ & Martha Entrekin & 1952 & D & 138 & $Q t$ & 80 & 1954 & $D, S$ \\
\hline J $34-14$ & H. G. Thompson & 1961 & $\mathrm{D}$ & 100 & Qt & 65 & $4-24-63$ & $\mathrm{D}$ \\
\hline J $36-4$ & Arthur Lowe & 1953 & B & 86 & Qt & 50 & $5-24-61$ & $\mathrm{D}, \mathrm{S}$ \\
\hline K 7-1 & R. L. Anderson, Sr. & 1949 & B & 98 & Qt & 76 & 1949 & $\mathrm{D}, \mathrm{S}$ \\
\hline K $7-4$ & G. W. Anderson & 1951 & B & 80 & Qt & 70 & 1951 & $\mathrm{D}, \mathrm{S}$ \\
\hline K $\quad 9-2$ & Miss. Forestry Comm. & 1959 & D & 131 & Qt & 80 & 1959 & $\mathrm{D}, \mathrm{S}$ \\
\hline K 18-1 & W. W. Beech & 1940 & B & 56 & Qt & 37.9 & $8-18-64$ & $\mathrm{D}, \mathrm{S}$ \\
\hline K $18-8$ & R. L. Anderson, Jr. & 1963 & $\mathrm{D}$ & 78 & Qt & -- & -- & $\mathrm{D}, \mathrm{S}$ \\
\hline K 28-1 & J. H. Busha & 1955 & $\mathrm{D}$ & 102 & Qt & -- & -- & $\mathrm{D}, \mathrm{S}$ \\
\hline K $33-2$ & Mrs. W. M. Courtney & 1936 & B & 45 & Qt & 30.4 & $4-23-65$ & $\mathrm{D}, \mathrm{S}$ \\
\hline L $16-2$ & Town of Purvis & 1961 & D & 984 & Tph & 214.0 & $10-9-61$ & PS \\
\hline o $31-1$ & Town of Lumberton & 1951 & $\mathrm{D}$ & 1,005 & Tc & 135 & $2-1-61$ & PS \\
\hline
\end{tabular}


Table 1.--Record of Lamar County, Mississippi, wells for which chemical analyses are included--Gontinued

\begin{tabular}{|c|c|c|c|c|c|c|c|c|}
\hline \multirow{2}{*}{$\begin{array}{l}\text { Wel1 } \\
\text { number }\end{array}$} & \multirow[b]{2}{*}{ Owner } & \multirow{2}{*}{$\begin{array}{l}\text { Year } \\
\text { drilled }\end{array}$} & \multirow{2}{*}{$\begin{array}{c}\text { Type } \\
\text { of } \\
\text { we11 }\end{array}$} & \multirow{2}{*}{$\begin{array}{l}\text { Depth } \\
\text { (ft) }\end{array}$} & \multirow{2}{*}{$\begin{array}{l}\text { Water- } \\
\text { bearing } \\
\text { unit }\end{array}$} & \multicolumn{2}{|c|}{ Water level } & \multirow{2}{*}{$\begin{array}{c}\text { Use } \\
\text { of } \\
\text { water }\end{array}$} \\
\hline & & & & & & $\begin{array}{c}\text { Below } \\
\text { LSD }\end{array}$ & $\begin{array}{c}\text { Date of } \\
\text { measurement }\end{array}$ & \\
\hline $\mathrm{HT}-2 \mathrm{c}$ & USAEC & 1961 & $D$ & 366 & Tph & 129.5 & $8-1-62$ & PS \\
\hline $\mathrm{HT}-4$ & USAEC & 1961 & $\mathrm{D}$ & 474 & Tph & 92.3 & $3-4-65$ & 0 \\
\hline $\mathrm{HT}-2 \mathrm{a}$ & USAEC & 1961 & $\mathrm{D}$ & 1,079 & Tph & 134.4 & $2-18-65$ & PS \\
\hline $\mathrm{HT}-5$ & USAEC & 1963 & $\mathrm{D}$ & 680 & Tph & 104.6 & $3-4-65$ & 0 \\
\hline $\mathrm{HT}-1 \mathrm{a}$ & USAEC & 1961 & $\mathrm{D}$ & 1,090 & Tph & 125.0 & $3-11-65$ & 0 \\
\hline $\mathrm{HT}-6$ & USAEC & 1963 & D & 812 & $\mathrm{Tph}$ & 108.8 & $3-12-65$ & 0 \\
\hline$E-1$ & USAEC & 1961 & $\mathrm{D}$ & 2,200 & Tc & 108.8 & $2-11-65$ & 0 \\
\hline $\mathrm{E}-14 \mathrm{~b}(\mathrm{U})$ & USAEC & 1963 & $\mathrm{D}$ & 1,075 & $\mathrm{Tc}$ & -- & -- & 0 \\
\hline $\mathrm{HT}-3$ & USAEC & 1963 & D & 1,064 & Tcc & 103.7 & $3-4-65$ & 0 \\
\hline$E-9$ & USAEC & 1961 & D & 1,789 & Tcc & 86.6 & $3-4-65$ & 0 \\
\hline$E-14 b(\mathrm{~L})$ & USAEC & 1963 & D & 1,075 & $\mathrm{Tcc}$ & -- & -- & 0 \\
\hline $\mathrm{HT}-1$ (3) & USAEC & 1961 & D & 2,402 & $\mathrm{Tc}$ & 149.6 & 1964 & 0 \\
\hline HT-2(3) & USAEC & 1961 & D & 2,622 & $\mathrm{Tc}$ & 137.5 & $9-13-65$ & 0 \\
\hline $\mathrm{HT}-1$ (4) & USAEC & 1961 & D & 2,402 & $\mathrm{Tv}$ & 127.6 & $12-2-64$ & 0 \\
\hline $\mathrm{HT}-2(4)$ & USAEC & 1961 & D & 2,622 & $\mathrm{Tv}$ & 120.0 & $9-3-65$ & 0 \\
\hline $\mathrm{HT}-1$ (5) & USAEC & 1961 & D & 2,402 & Tcm & 78.3 & $5-9-61$ & 0 \\
\hline $\mathrm{HT}-2(5)$ & USAEC & 1961 & $\mathrm{D}$ & 2,622 & $\mathrm{Tcm}$ & 85.0 & $6-30-61$ & Ind \\
\hline
\end{tabular}




\section{SUMMARY OF WELLS SAMPLED}

Except for the exploratory and hydrologic test wells, the wells are mostly shallow and completed in the sands and gravels of the local terrace deposits of Quaternary age which blanket most of the area under consideration. The deeper wells tap the underlying sands of Pascagoula and Hattiesburg Formations of Miocene age. Some of the hydrologic test wells are completed in even deeper aquifers, among which are the Catahoula Sandstone, the limestones of the Vicksburg Group, and the Cook Mountain Limestone.

\section{WATER QUALITY}

As a part of studies before and after the Salmon Event, samples were collected to provide a base of comparison of any possible waterquality changes that might be attributed to the Salmon Event experiment. No description of the water quality is being attempted in this data release as a comprehensive review and description of water quality in the area by Beetem and others will be released shortIy. The water analyses are identified as coming from aquifers; for example, terrace deposits, Catahoula Sandstone, etc.

To complete the release of data in our files, the radiochemical analyses of gross beta, gross alpha, and tritium follow the chemical analyses for the wells not in the immediate vicinity of the Tatum salt dome. The radiochemical data in the immediate vicinity has been previously released. The tritium values reported are sought but not detected by method used. The method used was liquid scintillation counting and most probable limit of detection for these samples was $2 \times 10^{-6} \mu \mathrm{c} / \mathrm{ml}$ (microcuries per milliliter). Although this concentration is less than might 
be expected on samples of rainfall, it is much higher than normal ground... water concentrations; so little significance can be attached to these values, other than that the waters are not grossly contaminated.

\section{RADIOCHEMICAL ANALYSES}

Water samples received for analysis at the Denver Laboratory are first assigned a laboratory number. The sample bottles are then marked with these numbers and all pertinent information supplied with the sample is typed on data cards. All identifying information written on the bottle is removed before the sample is submitted to the analysts for analysis. These procedures have been adopted as a quality-control measure and are standard for all incoming water samples.

Gross alpha, gross beta, and tritium determinations are made on all samples submitted. When anomalously high gross beta or gross alpha counts are obtained, sample planchets are recounted and a duplicate sample prepared to substantiate the data. If the duplicate tritium determinam tion indicates concentrations near normal background Ievels but with discrepancy of data, aliquots of the samples are sent to a laboratory in Washington, D.C., for electrolytic concentration and subsequent low-1evel liquid scintillation counting. Various other radiochemical determinations may be made, depending on the results obtained for gross alpha and gross beta determinations and a gross gamma scan.

The determination of the tritium concentration is made on part of: the distillate obtained from a small volume of the sample, which is dism tilled to dryness. For counting, a 4.0-m1 (milliliter) aliquot of the sample distillate is mixed with $18.0-\mathrm{ml}$ aliquot of a dioxane-base liquid 
scintillation "cocktail" in a polyethylene counting vial. The vial is placed in a refrigerated sample changer and associated Packard Model $314 \mathrm{E}$ liquid scintillation spectrometer, and the sample is counted three times over a period of 2 days. The entire procedure is repeated after an interval of at least 7 days and the six values averaged and reported.

Beta activity of the water samples analyzed is determined on sample aliquots ranging in volume from 5 to $1,000 \mathrm{ml}$. Aliquot volumes chosen for analyses are dependent upon the total dissolved solids in the samples and are chosen to yield deposits within certain weight tolerances, which will standardize self-absorption of the samples.

Gross alpha values are reported for aliquots of sufficient size to give significant counts above background. For aliquots too small to give significant counts above background, gross aIpha values are reported as "sought but not detected by method used."

\section{CHEMICAL ANALYSES}

In this study the chemical analyses basically consisted of the determination of silica $\left(\mathrm{SiO}_{2}\right)$, aluminum (A1), iron ( $\left.\mathrm{Fe}\right)$, manganese (Mn), calcium (Ca), magnesium $(\mathrm{Mg})$, strontium $(\mathrm{Sr})$, sodium $(\mathrm{Na})$, potassium (K), Iithium $(\mathrm{Ii})$, copper $(\mathrm{Cu})$, zinc $(\mathrm{Zn})$, bicarbonate $\left(\mathrm{HCO}_{3}\right)$, carbonate $\left(\mathrm{CO}_{3}\right)$, sulfate $\left(\mathrm{SO}_{4}\right)$, chloride (C1), fluoride $(\mathrm{F})$, nitrate $\left(\mathrm{NO}_{3}\right)$, phosphate $\left(\mathrm{PO}_{4}\right)$, dissolved solids, hardness, specific conductance, pH, color, and temperature.

The values of most constituents obtained by chemical analysis represent: the amount of the constituents in solution at the time of the analysis. For some constituents and properties, these values may be slightly higher or lower than the values that might have been found if the analysis had been done directly at the sampling site. 
The principal methods used in the chemical analysis of water samples reported herein were analyzed as described in the following paragraphs. For a more thorough description of the methods used, the reader is referred to "Methods for Collection and Analysis of Water Samples," by Rainwater and Thatcher (1960).

Generally, pH is measured by immersing a set of electrodes in a water sample; the potential produced is measured by a $\mathrm{pH}$ meter。 Silica, iron, aluminum, nitrate, fluoride, phosphate, and sulfate are determined by adding a known amount of color-causing reagent to water and measuring the intensity of the resultant color in a spectrophotometer. The intensity of the color produced is approximately proportional to the concentration of the constituent being determined.

Hardness, chloride, carbonate, and bicarbonate are determined by adding a measured amount of standardized reagent to a known volume of water until a color change or a $\mathrm{pH}$ change signals that the reaction is complete.

Sodium, potassium, lithium, manganese, calcium, magnesium, strontium, copper, and zinc are measured in an atomic absorption photometer. In this determination, the solution to be analyzed is vaporized in a flame; then the resultant color intensity of the flame is compared to the emission of the same metal from a standard lamp.

All these methods mentioned require a fairly low concentration of the constituent being analyzed; the samples of water analyzed, with the exception of the samples from the Cook Mountain Limestone, fall in this category. For those samples, some of the constituents were measured 
gravimetrically. In this method, the substance to be analyzed is precipitated; the precipitate is filtered, washed, and weighed. This method, depending on the sample, is at times used to determine silica, calcium, magnesium, sodium, sulfate, and chloride.

Dissolved solids are usually measured by evaporating a known volume of water sample, drying the residue at $180^{\circ} \mathrm{C}$ for 1 hour, cooling the residue in a desiccator, and then weighing the residue. Most of the chemical analyses in this report give dissolved solids determined by the evaporation method and by calculation.

Specific conductance of water is determined by dipping a cell into a water sample and measuring its specific electrical resistance. The specific conductance of the water is the reciprocal of the specific resistance of the water and is reported in micromhos (onemillionth of a mho). 


\section{REFERENCES CITED}

Beetem, W. A., and Grove, D. B., 1971a, Radiochemical analyses of ground-water samples collected after the Salmon Event in the vicinity of Tatum salt dome, Mississippi: U.S. Geo1. Survey rept. USGS-474-124, 48 p.; available only from U.S. Dept. Commerce, Natl. Tech. Inf. Service, Springfield, Va, 22151.

1971b, Radiochemical analyses of ground-water and surface-water samples collected after the Salmon Event in the vicinity of Tatum salt dome, Mississippi: U.S. Geol. Survey rept. USGS-474-119, 76 p.; available only from U.S. Dept. Commerce, Nat1. Tech. Inf. Service, Springfield, Va. 22151.

Janzer, V. J., Robinson, B. P., and Rucker, S. J., 1971a, Radiochemical analyses of water samples collected after the Salmon Event in the vicinity of Tatum salt dome, Mississippi: U.S. Geol. Survey rept. USGS-474-115, 32 p.; available only from U.S. Dept. Commerce, Nat1。Tech. Inf. Service, Springfield, Va. 22151.

1971b, Radiochemical analyses of ground-water samples collected after the Salmon Event in the vicinity of Tatum salt dome, Mississippi: U.S. Geol. Survey rept. USGS-474-117, 86 p.; available only from U.S. Dept. Commerce, Nat1. Tech. Inf. Service, Springfield, Va. 22151.

Rainwater, F。 H., and Thatcher, L. L., 1960, Methods for the collection and analysis of water samples: U.S. Geo1. Survey Water-Supply Paper 1454, 301 p. 


\section{U. S. DEPT. OF THE INTERIOR -- GEOLOGICAL SURVEY \\ Statement of Water Analysis}

Source Grantham Creek at Well E-9

Location Tatum Salt Dome 10 miles west of Purvis, Lamar County, Mississippi

${ }^{1 / 4}{ }^{1 / 4}{ }^{1 / 4} \mathrm{Sec}-13, T^{\top} 2 \mathrm{Ne}, \mathrm{R} 16 \mathrm{~W}$. Field/Office No.

Date Col. 9-9-65 Time_1330_ Well Type

Col. By USGS

Field detns: Temp. $\left({ }^{\circ} \mathrm{F}\right)$

Sp. Cond. (umhos)

Appearance $\mathrm{pH}$

Depth ( $f t$.)

Diam. (in.)

Water level ( $f t$.

Discharge

W. B. F.

Owner

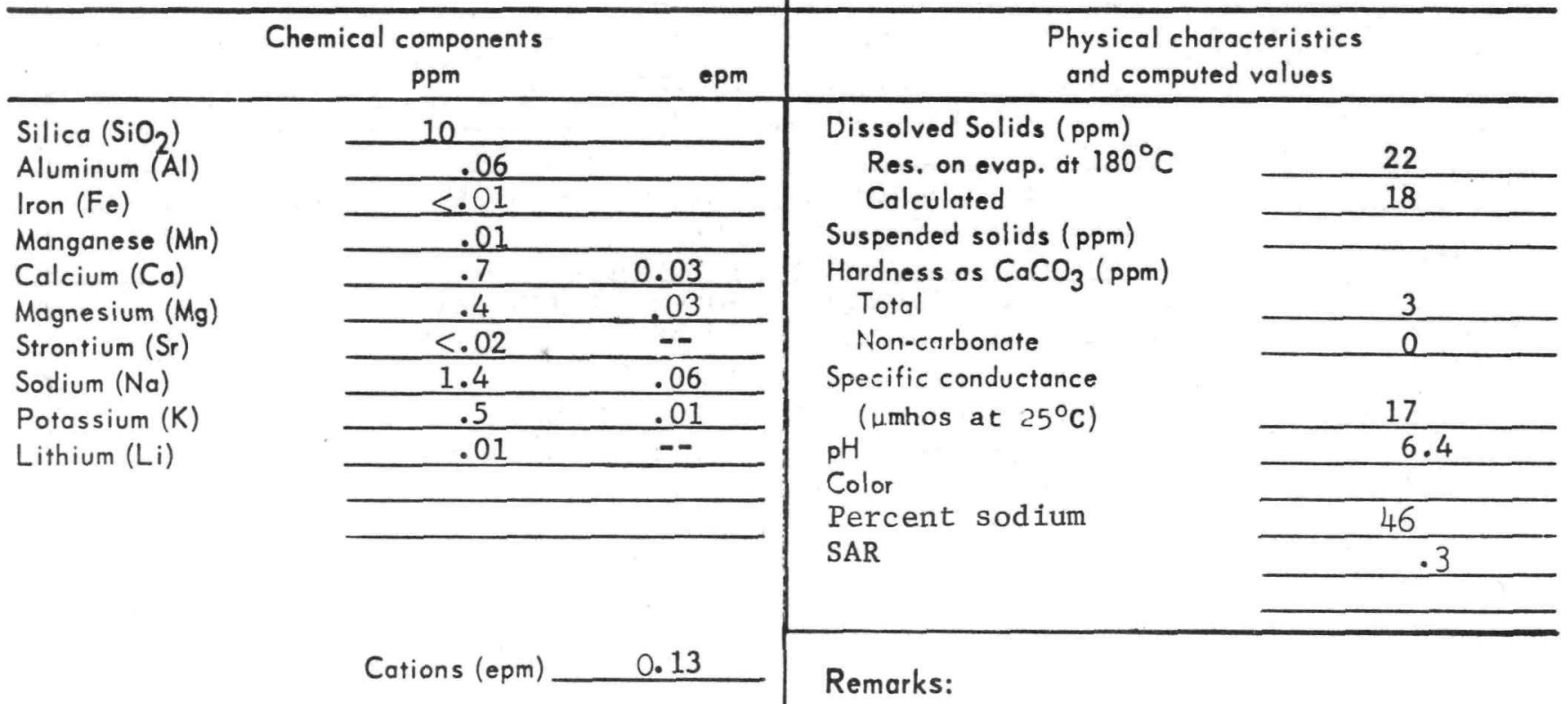

Bicarbonate $\left(\mathrm{HCO}_{3}\right)$

Carbonate $\left(\mathrm{CO}_{3}\right)$

Sulfate $\left(\mathrm{SO}_{4}\right)$

Chloride (CI)

Fluoride (F)

Nitrate $\left(\mathrm{NO}_{3}\right)$

Phosphate $\left(\mathrm{PO}_{4}\right)$ clear
Use

Cased to

Date drilled
Eh

$-$

Remarks:

Dissolved Solids (ppm)

Res. on evap. at $180^{\circ} \mathrm{C}$

ardness os $\mathrm{CaCO}_{3}$ (ppm)

Total

Non-carbonate

Specific conductance

(umhos at $25^{\circ} \mathrm{C}$ )

Color

Percent sodium

SAR

0.08

\begin{tabular}{cc}
5 & 0.08 \\
\hline 0 & .00 \\
\hline$<.1$ & -- \\
\hline 2.6 & .07 \\
\hline .1 & .00 \\
\hline .1 & .00 \\
\hline$<.01$ & -- \\
\hline
\end{tabular}




\section{U. S. DEPT. OF THE INTERIOR -. GEOLOGICAL SURVEY \\ Statement of Water Analysis}

Source Half Moon Creek, P/C: at gaging station

Location See Remarks

SW $1 / 4-1 / 4-1 / 4$ Sec 2, T T 2 Ne, R $16 \mathrm{~W}$. Field/Office No.

Date Col._9-9-65_Time_1340_U Use

Col. By USGS Depth (ft.)

Field detns: Temp. $\left({ }^{\circ} \mathrm{F}\right) \_72 \quad \mathrm{pH} \_$Diam. (in.)

Cased to

Diam. (in.) Date drilled

Sp. Cond. (umhos)

Appearance

Clear

Water level (ft.)

Discharge

W. B. F.

Drainage area (sq. miles): Approx. 20

Owner

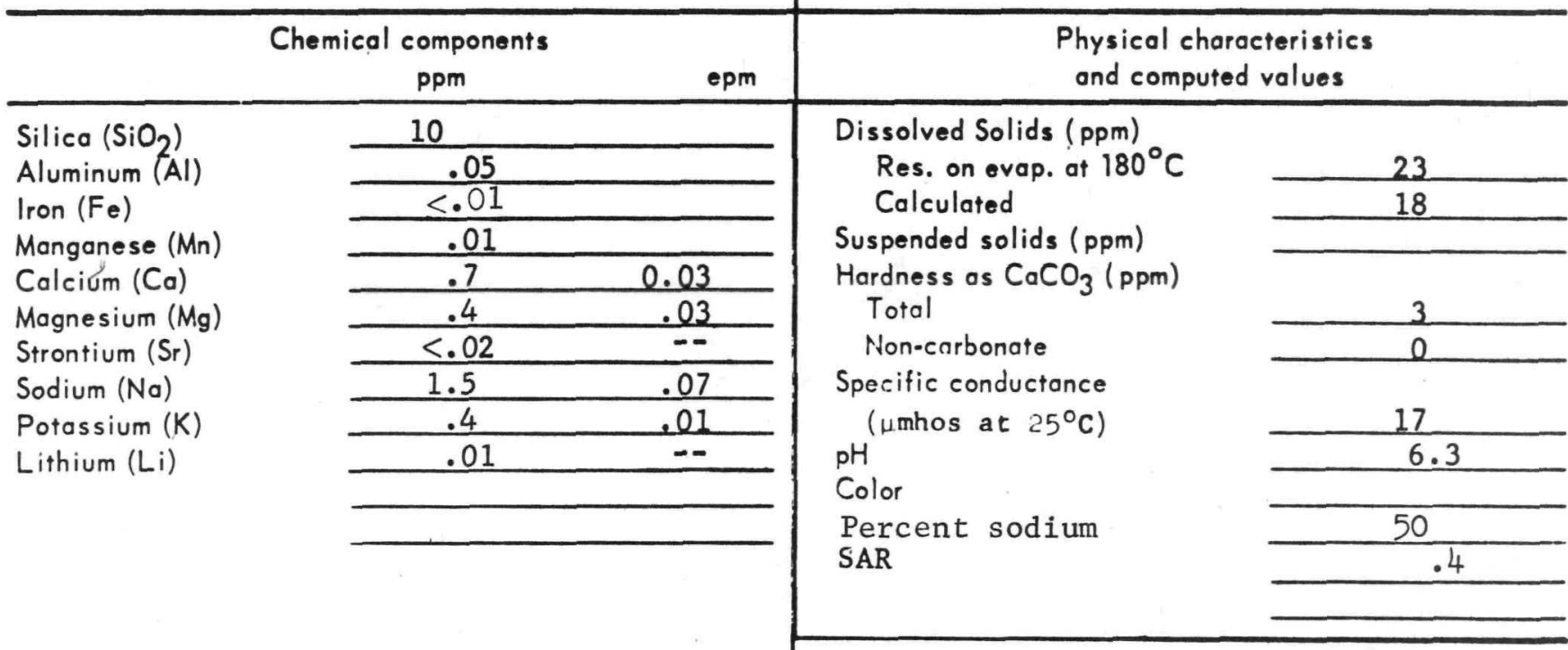

\section{Cations (epm)}

Bicarbonate $\left(\mathrm{HCO}_{3}\right)$

Carbonate $\left(\mathrm{CO}_{3}\right)$

Sulfate $\left(\mathrm{SO}_{4}\right)$

Chloride (Cl)

Fluoride (F)

Nitrate $\left(\mathrm{NO}_{3}\right)$

Phosphate $\left(\mathrm{PO}_{4}\right)$

\begin{tabular}{cc}
5 & 0.08 \\
\hline 0 & .00 \\
\hline. .1 & .00 \\
\hline 2.4 & .07 \\
\hline$<.1$ & .00 \\
\hline .1 & .00 \\
\hline$<.01$ & -- \\
\hline
\end{tabular}

Remarks:

Location: Near Baxterville, Lamar Co., Mississippi, 8 miles west of Purvis, off Purvis Salt Dome Road.

St. Stephens Meridian immediately upstream from confluence with Hurrican Creek and $5 \frac{1}{2}$ miles north of Baxterville. 


\section{U. S. DEPT. OF THE INTERIOR - GEOLOGICAL SURVEY \\ Statement of Woter Analysis}

Source Lower Little Creek P/C: at gaging station

Location See Remarks

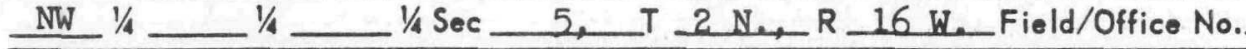

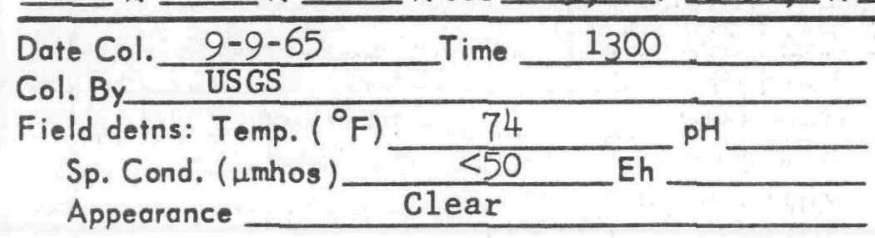

Well Type

Depth (ft.)

Diam. (in.)

Water level (ft.)

Discharge

W. B. F.

Drainage area (sq. miles): Approx. 80

Owner

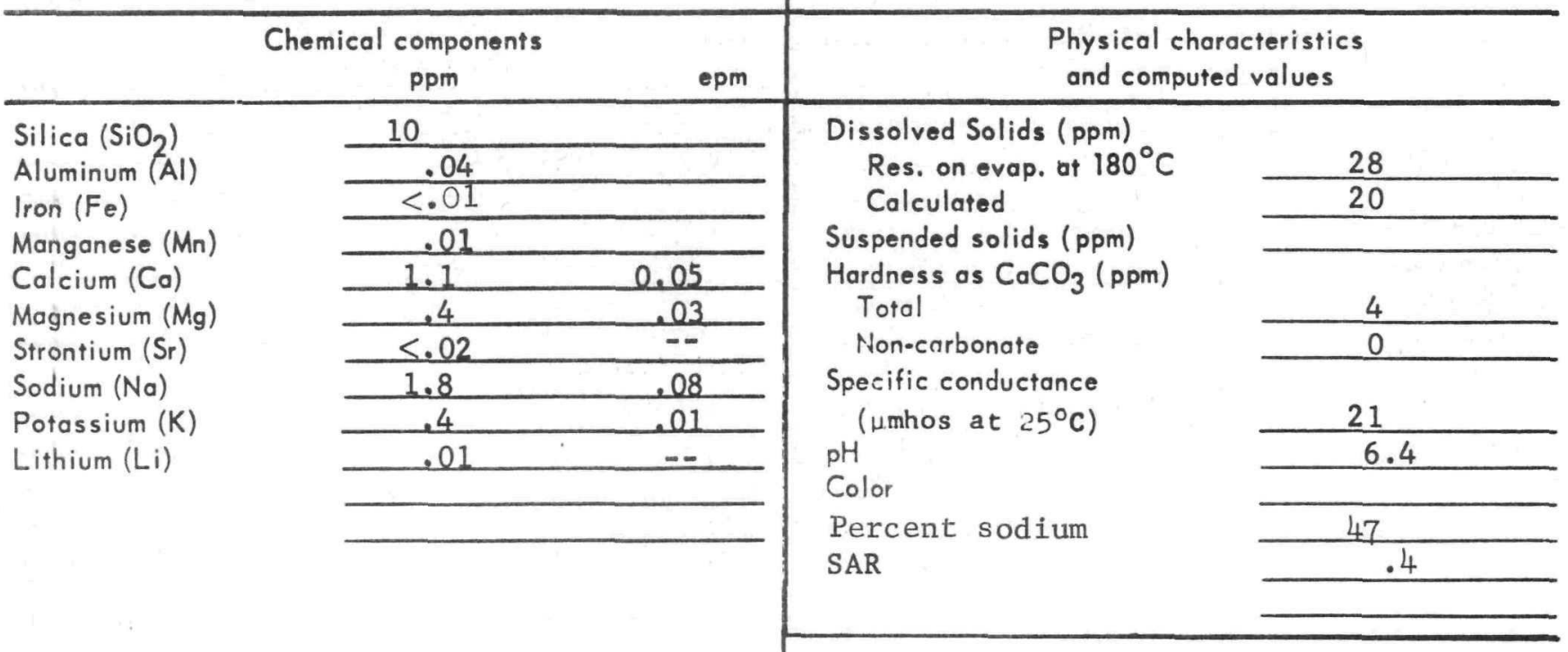

\section{Cations (epm) 0.17 Remarks:}

Bicarbonate $\left(\mathrm{HCO}_{3}\right)$

Carbonate $\left(\mathrm{CO}_{3}\right)$

Sulfate $\left(\mathrm{SO}_{4}\right)$

Chloride (CI)

Fluoride ( $F$ )

Nitrate $\left(\mathrm{NO}_{3}\right)$

Phos phate $\left(\mathrm{PO}_{4}\right)$

\begin{tabular}{cc}
6 & 0.10 \\
\hline 0 & .00 \\
\hline$<.1$ & -- \\
\hline 2.9 & .08 \\
\hline$<.1$ & .00 \\
\hline .1 & .00 \\
\hline$<.01$ & -- \\
\hline
\end{tabular}

Location: Near Baxterville, Lamar Co., Mississippi, 11 miles west of Purvis, off Purvis Salt Dome Road.

Anions (epm) $\quad 0.18$ 


\section{U. S. DEPT. OF THE INTERIOR -- GEOLOGICAL SURVEY \\ Statement of Water Analysis}

Source We11 F25-2

Location Lumberton, Lamar Co., Mississipp1

SW $1 / 4 \ldots 1 / 4 \ldots$ Sec $-25, T \cdot 3$ N., R. 16 W. Field/Office No.

Date Col._10-25-65 Time_1600_ Woll Type Bored Use Dom; stock

Col. By_USGS

Field detns: Tomp. $\left({ }^{\circ} \mathrm{F}\right) \quad 68$

Sp. Cond. (umhos)

Appearance

Eh

Clear

Depth $(f t) \quad$.

Diam. (in.)

Cased to Unknown

Woter lovel ( $f \mathrm{f}$.) Unknown

Discharge $2.5 \mathrm{gpm}$ on $5-24-61$

W. B. F. C1tronelle $\mathrm{Fm}$.

Owner Buel Bryant,

Rt. 4, Lumberton, Miss.

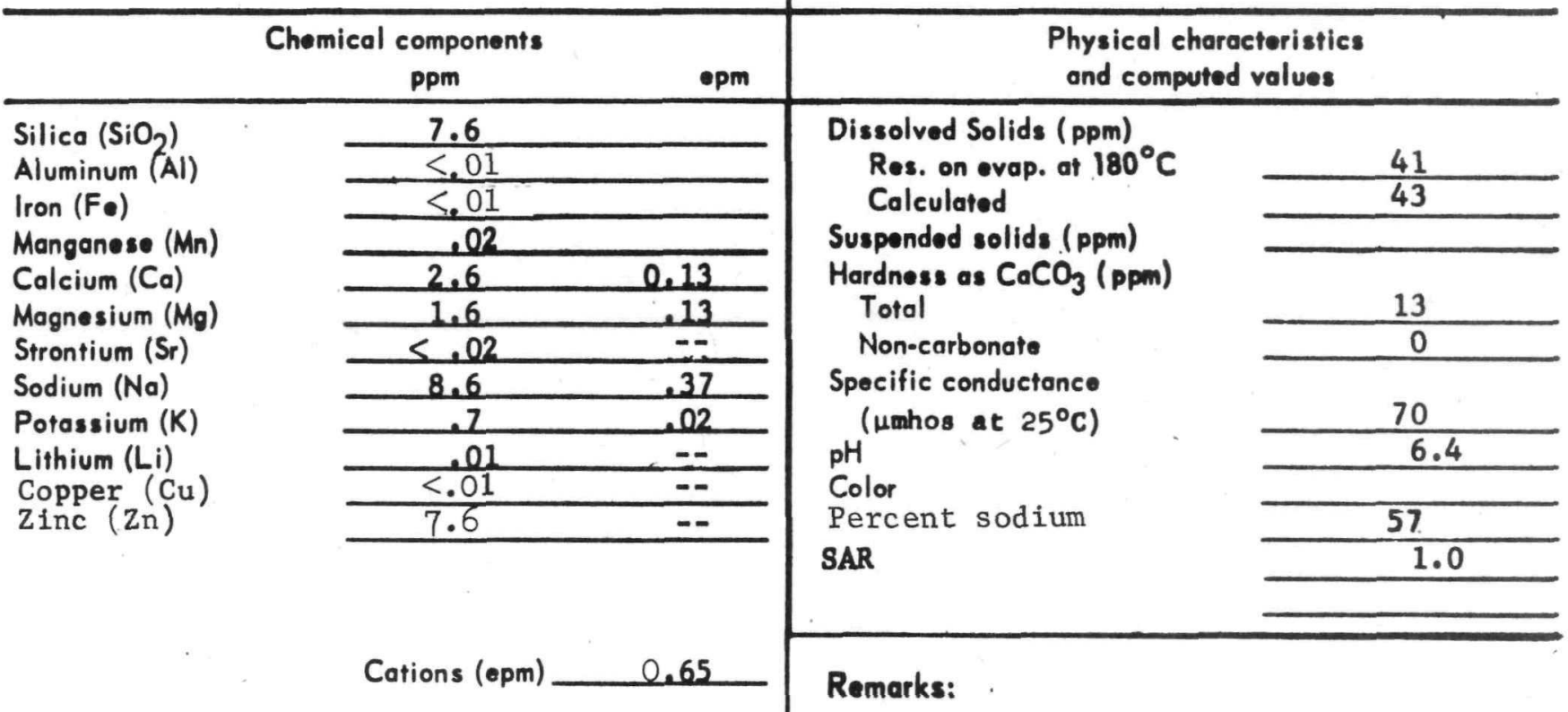

Bicarbonate $\left(\mathrm{HCO}_{3}\right)$
Carbonate $\left(\mathrm{CO}_{3}\right)$
Sulfate $\left(\mathrm{SO}_{4}\right)$
Chloride $(\mathrm{CI})$
Fluoride $(\mathrm{F})$
Nitrate $\left(\mathrm{NO}_{3}\right)$
Phosphate $\left(\mathrm{PO}_{4}\right)$

\begin{tabular}{cc}
34 & 0.56 \\
\hline 0 & .00 \\
\hline 1.0 & .02 \\
\hline 2.9 & .08 \\
\hline 5.1 & -- \\
\hline .9 & .01 \\
\hline$<.01$ & --- \\
\hline & \\
\hline
\end{tabular}

Well has $4.5 \mathrm{ft}$. stralner.

Anions (epm) 0.67

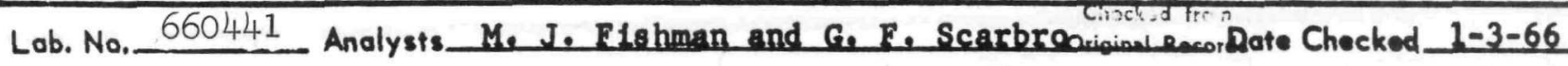
QW - 1A 
U, S. DEPT. OF THE INTERIOR--GEOLOGICAL SURVEY

Statement of Water Analysis - Radiochemical

Source

We11 F25-2

Location Lumberton, Lamar Co., Mississippi
SW $1 / 4$
$1 / 4$
$1 / 4 \mathrm{sec} \cdot 25$, T. 3 N.,
R. I6 W. Field/Office No.

Date Col. 10-25-65 Time 1600

Col. By USGS

Field detns: Temp. (OF) 68

Sp. Cond. ( $\mu$ mhos)

Appearance

Clear

$\mathrm{Eh}^{\mathrm{pH}}$




\section{U. S. DEPT. OF THE INTERIOR -. GEOLOGICAL SURVEY Statement of Water Analysis}

Source Well F33-2

Location Lumberton, Lamar Co., Mississippi

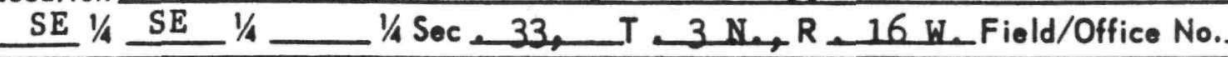

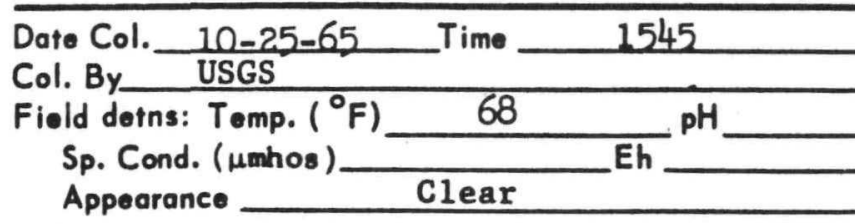

\begin{tabular}{c|c}
\hline $\begin{array}{c}\text { Chemical components } \\
\text { ppm }\end{array}$ & epm \\
\hline 52 &
\end{tabular}

Silica $\left(\mathrm{SiO}_{2}\right)$

Aluminum (Al)

Iron (Fo)

Manganese (Mn)

Calcium (Ca)

Magnesium (Mg)

Strontium (Sr)

Sodium ( $\mathrm{Na}$ )

Potassium (K)

Lithium (Li)

Copper (Cu)

Zinc $(\mathrm{Zn})$

\begin{tabular}{cc}
\multicolumn{2}{l}{52} \\
\hline$<.01$ \\
\hline$<.01$ \\
\hline$<.02$ \\
\hline 22 & \\
\hline 3.0 & 1.10 \\
\hline .25 & .25 \\
\hline 27 & -- \\
\hline 2.9 & 1.17 \\
\hline .08 & .07 \\
\hline$<.01$ & -- \\
\hline .50 & -- \\
\hline
\end{tabular}

Dissolved Solids (ppm)

Res. on evap. of $180^{\circ} \mathrm{C}$

Calculated

Suspended solids (ppm)

Hardness os $\mathrm{CaCO}_{3}$ (ppm)

Total

Non-carbonate

Specific conductance

( $\mu$ mhos at $25^{\circ} \mathrm{C}$ )

$\mathrm{pH}$

Color

Percent sodium

SAR

Cations (epm) $\quad 2.60$
Bicarbonate $\left(\mathrm{HCO}_{3}\right)$

Carbonate $\left(\mathrm{CO}_{3}\right)$

Sulfate $\left(\mathrm{SO}_{4}\right)$

Chloride $(\mathrm{Cl})$

Fluoride (F)

Nitrate $\left(\mathrm{NO}_{3}\right)$

Phosphate $\left(\mathrm{PO}_{4}\right)$

\begin{tabular}{cc}
134 & 2.20 \\
\hline 0 & .00 \\
\hline$<.1$ & -- \\
\hline 11 & .31 \\
\hline .1 & .01 \\
\hline$<.1$ & -- \\
\hline$<.01$ & -- \\
\hline & \\
\hline & \\
\hline
\end{tabular}

Anions (epm) 2.52

Anions (epm) 2.52

Remarks:
188

184
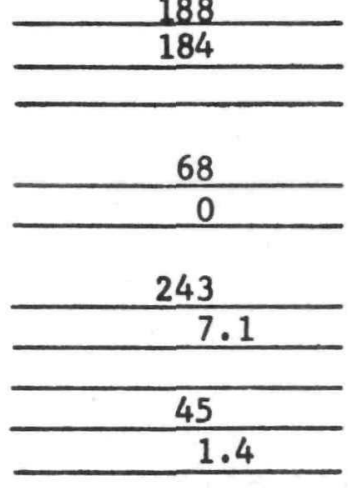

Use Dom; stock

Cased to $225 \mathrm{ft}$.

Date drilled $\frac{1951}{1951}$

Discharge $1 \mathrm{gpm}$ on $5-16-63$ undiff. Rt. 4, Lumberton, Miss.

Physical characteristics and computed values

$\begin{array}{r}188 \\ \hline 184 \\ \hline \\ \hline 68 \\ \hline 0 \\ \hline 243 \\ \hline 7.1 \\ \hline 45 \\ \hline 1.4 \\ \hline\end{array}$

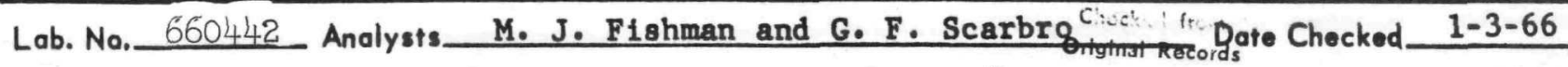

QW - 1A 
U. S. DEPT. OF THE INIERIOR--GEOLOGICAL SURVEY

Statement of Water Analysis - Radiochemical

Source

Wel1 F33-2

Location Lumberton, Lamar Con Mississippi

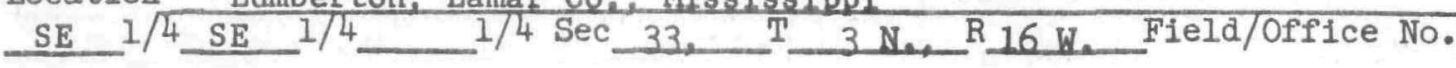

2000

Date Col. 10-25-65 Time 1545

Sp. Cond. ( $\mu$ mhos)

Appearance
68 Eh.

Clear
Col. By USGS

Field detns: Temp. ( ${ }^{\circ} \mathrm{F}$ )

Well Type Drilled

Depth $(\mathrm { ft } \longdiv { 2 3 0 }$

Cased to $225 \mathrm{ft}$.

Diam. (1n) 2 Date Drilled 1951

Water level (ft) $80+$ below 1.s.d., 1951

Discharge 1 gpm on $5-16-63$

W.B.F. Pascagoula and Hattiesburg Fms., undiff.

Owner Sara Entrekin, Rt. 4, Lumberton, Miss.

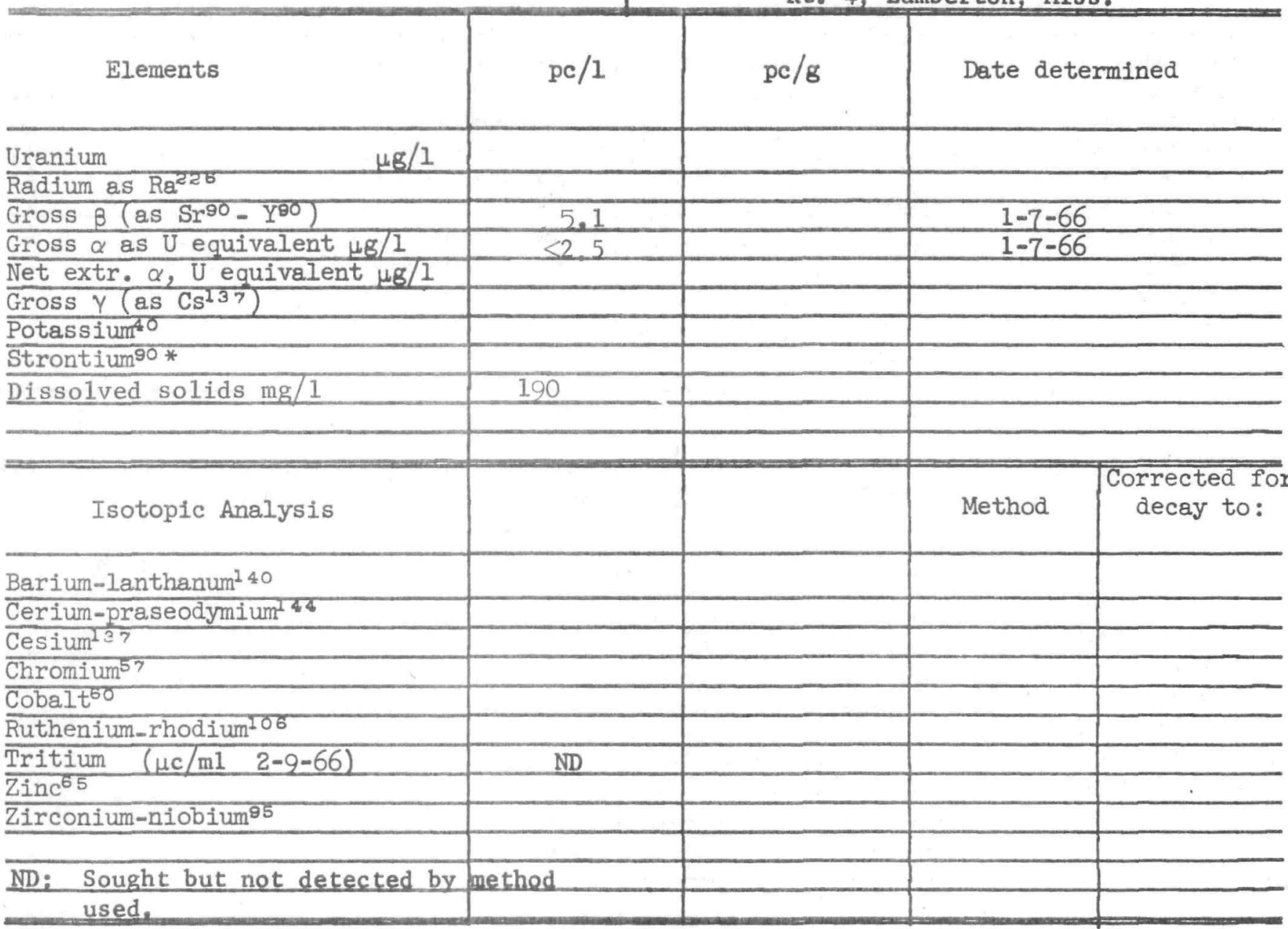

Date sample received 12-14-65

Date data released 1-17-66

Program classification

Analyst T.Curran; P.K.Roscio; H.C.Claassen Date checked 1-17-66

Checked by J. J. Janzer

*Analyzed only when indicated by gross $\beta$ count.

Revised form 3-17-65

20

Checked from

Original Records

Ay $2-22=66$

$6 P O B 48 \cdot 788$ 


\section{U. S. DEPT. OF THE INTERIOR -- GEOLOGICAL SURVEY Statement of Water Analysis}

Source Well F36-5

Location Lumberton, Lamar Co., Mississippi

NE $1 / 4$ NW $1 / 4 \ldots 1 / 4$ Sec $36, T, 3 N, R, 16$ W. Field/Office No.

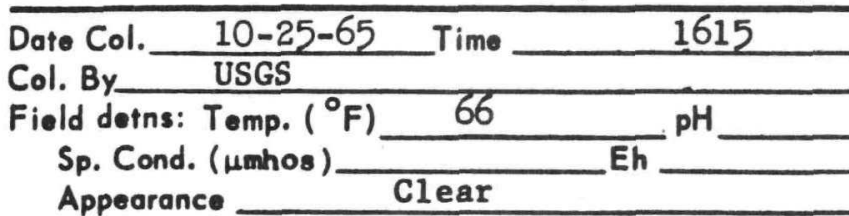

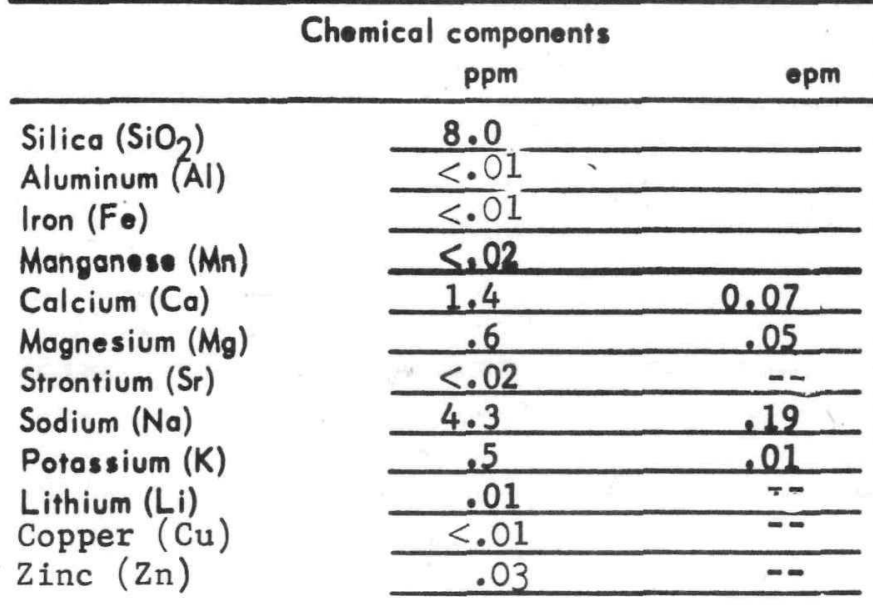

Well Type Bored

Depth (ft.)

Diam. (in.)

Water lovel (ff.) 67 (below 1.s.d.)

Discharge 2 gallons, $17 \mathrm{sec}$, , 5-25-61

W. B. F. Citronelle Fm.

Owner J. C. Gibson, Rt. 4, Lumberton, Miss.

Physical characteristics and computed values

\section{Dissolved Solids (ppm)}

Res. on evap. of $180^{\circ} \mathrm{C}$

Calculated

Uso Dom; stock

Cased to See Remarks

Date drilled 1949

uspended solids (ppm)

Hardness as $\mathrm{CaCO}_{3}(\mathrm{ppm})$

Total

Non-carbonate

Specific conductance

( $\mu$ mhos at $25^{\circ} \mathrm{C}$ )

$\mathrm{pH}$

Color

Percent sodium

SAR

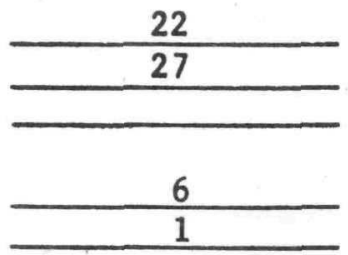

$\begin{array}{r}29 \\ \hline 6.6 \\ \hline 59 \\ \hline .8\end{array}$

Cations (epm)

0.32

Bicarbonate $\left(\mathrm{HCO}_{3}\right)$

Carbonate $\left(\mathrm{CO}_{3}\right)$

Sulfate $\left(\mathrm{SO}_{4}\right)$

Chloride $(\mathrm{Cl})$

Fluoride ( $F$ )

Nitrate $\left(\mathrm{NO}_{3}\right)$

Phosphate $\left(\mathrm{PO}_{4}\right)$

\begin{tabular}{lc}
6 & 0.10 \\
\hline 0 & .00 \\
\hline 1.0 & .02 \\
\hline 4.3 & .12 \\
\hline$<.1$ & .00 \\
\hline 4.0 & .06 \\
\hline$<.01$ & -- \\
\hline \multicolumn{2}{l}{} \\
\hline
\end{tabular}

Remarks:

Cased to: Tile well with jet pump installed within.

Anions (epm) $\quad 0.30$

Lab. No. 660443 Analysts

QW - IA
M. J. Fishman and

1

1


U. S. DEPT. OF THE INIERIOR--GEOLOGICAL SURVEY

Statement of Water Analysis - Radiochemital

Source Wel1 F36-5

Location Lumberton, Lamar Co., Mississippi $\mathrm{NE} 1 / 4 \mathrm{NW} 1 / 4 \ldots 1 / 4 \mathrm{Sec} .36, \mathrm{~T} \cdot 3 \mathrm{~N} .2 \mathrm{R} \cdot 16 \mathrm{~W}$. Field/Office No.

Date Col. 10-25-65 Time 1615 Well Type Bored Use Dom; stock

Col. By USGS Depth (ft 75 Cased to See Remarks

Field detas: Temp. (oF) $66 \quad$ Diam. (1n) 8 Date Drilled 1949

Sp. Cond. ( $\mu$ mhos)_ Wh _ Water level (ft) 67 (below 1.s.d.?)

Appearance Clear

Discharge 2 gallons, $17 \mathrm{sec}$, 5-25-61

W.B.F. Citronelle Fm.

\begin{tabular}{c}
$\frac{\text { Ower_ J. C. Gibson, }}{\text { Rt. 4, Lumberton, Miss. }}$ \\
\hline
\end{tabular}

\begin{tabular}{|c|c|c|c|}
\hline Elements & $\mathrm{pc} / 1$ & $\mathrm{pc} / \mathrm{g}$ & Date determined \\
\hline $\begin{array}{l}\text { Uranium } \\
\text { Radium as Ra }\end{array}$ & & & $\cdot$ \\
\hline Gross $\beta\left(\right.$ as $\left.\mathrm{Sr}^{90}-\mathrm{Y}^{80}\right)$ & 2.0 & & $2-5-66$ \\
\hline Gross $\alpha$ as U equivalent $\mu g / 1$ & 1.2 & & $2-5-66$ \\
\hline \multicolumn{4}{|l|}{ Net extr. $\alpha, \mathrm{U}$ equivalent $\mu \mathrm{g} / \mathrm{l}$} \\
\hline \multicolumn{4}{|l|}{ Gross $Y\left(\right.$ as $\left.\operatorname{Cs}^{137}\right)$} \\
\hline \multicolumn{4}{|l|}{ Potassium 40} \\
\hline \multicolumn{4}{|l|}{ Strontium $90 *$} \\
\hline Dissolved solids mg/1 & 26 & & \\
\hline & & & \\
\hline
\end{tabular}

\section{Isotopic Analysis}

Barium-lanthanum 140

Cerium-praseodymium ${ }^{16}$

Cesium ${ }^{137}$

Chromium ${ }^{5}$ ?

Cobalt

Ruthenium-rhodium ${ }^{106}$

Tritium

Zinc $^{65}$

Zirconium-niobium ${ }^{95}$

\begin{tabular}{l|l|l|}
\hline & \\
\hline & \\
\hline
\end{tabular}

Date sample received 12-14-65

Date data released 2-11-66

Program classification

*Analyzed only when indicated by gross $\beta$ count.

Revised form 3-17-65
22
Remarks :

Cased to: Tile well with a jet pump installed.

ND: Sought but not detected by method used $2-9-66$.
Analyst P.K.Roscio; T.Curran; H.C.Claassep Date checked Checked by $2-11-66$ V. J. Janzer

Checked from

Original Records

By..

Date $2-2 z-66$ 


\section{U. S. DEPT. OF THE INTERIOR .. GEOLOGICAL SURVEY}

Statement of Water Analysis

Source We11 J1-1

Location Lumberton, Lamar Co., Mississipp1

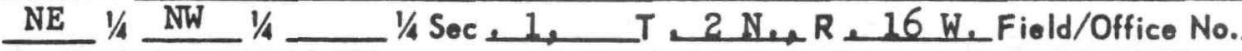

Date Col. 10-25-65_Time_1630 Woll Type Drilled_Use_Dom; stock

Col. By USGS

Field detns: Temp. $\left({ }^{\circ} \mathrm{F}\right) \quad 70 \quad \mathrm{pH}$

Sp. Cond. (umhos)

Appearance

Clear

Depth (ft.) 120

Diam. (in.) 2 Date drilled Unknown

Water lovel (ft.) $29 \mathrm{ft}$. below 1.8.d.e 1961

Discharge 3 8pm, 8-19-64 (measured)

W. B. F. Quaternary (alluvium, terrace deposits, etc.)

Owner Horace W. Burge, Rt. 4, Lumberton, Miss.

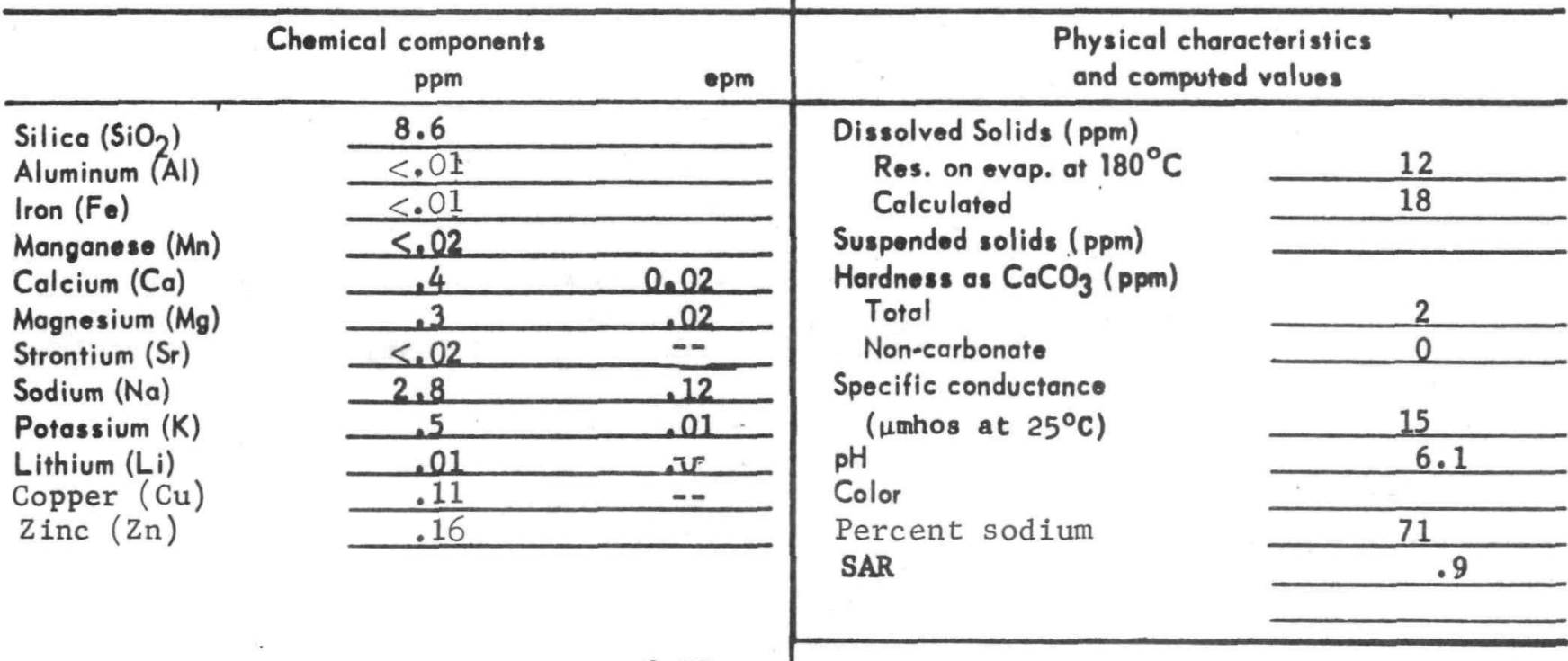

Cations (epm)

Bicarbonate $\left(\mathrm{HCO}_{3}\right)$

Carbonate $\left(\mathrm{CO}_{3}\right)$

Sulfate $\left(\mathrm{SO}_{4}\right)$

Chloride (CI)

Fluoride (F)

Nitrate $\left(\mathrm{NO}_{3}\right)$

Phosphote $\left(\mathrm{PO}_{4}\right)$

\begin{tabular}{cc}
5 & 0.08 \\
\hline 0 & .00 \\
\hline$<.1$ & -- \\
\hline 2.9 & .08 \\
\hline$<.1$ & -- \\
\hline .4 & .01 \\
\hline$<.01$ & -- \\
\hline \multicolumn{2}{r}{} \\
\hline \multicolumn{2}{c}{} \\
\hline
\end{tabular}

Anions (epm) 0.17

Anions (epm) $\quad 0.17$

Lab. Na. 660444 Analysts

QW - IA
M. J. ELahman an d G. F. Scarbrg Checked froDat

Bate..2 $-27-66$ 
U. S. DEPT. OF THE INTERIOR--GEOLOGICAL SURVEY

Statement of Water Analysis - Radiochemical

Source Wel1 J1-1

Location Lumberton, Lamar Co., Mississippi

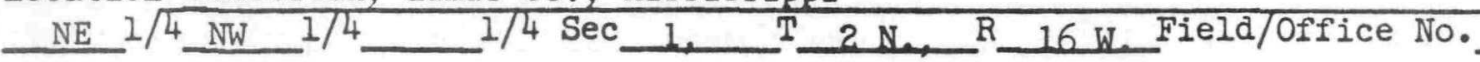

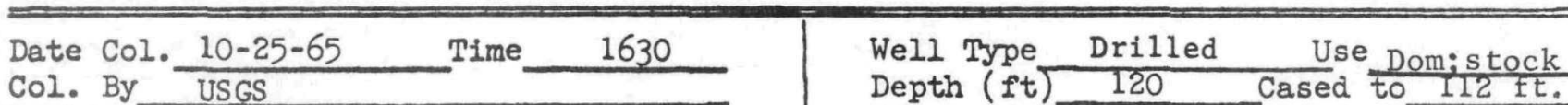

Col. By USGS Depth ( ft) 120 Cased to $112 \mathrm{ft}$.

Field detns: Temp. (PF) $70 \quad$ pH__ Diam. (in) 2 Date Drilied_Unknown

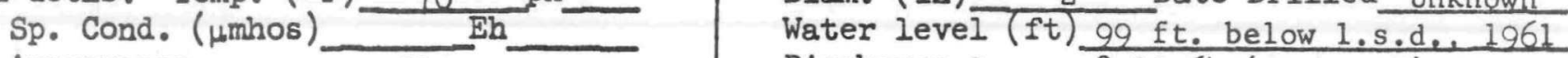

Appearance clear

Discharge $3 \mathrm{gpm}, 8-19-64$ (measured)

W.B.F. Quaternary (alluvium, terrace deposits. etc.)

Owner Horace W. Burge, Rt. 4, Lumberton, Miss.

\begin{tabular}{|c|c|c|c|c|}
\hline Elements & $\mathrm{pc} / 1$ & $\mathrm{pc} / \mathrm{g}$ & Date d & Tnined \\
\hline Uranium $\mu g / 1$ & & & & \\
\hline Radium as $\mathrm{Ra}^{\mathrm{C}} \mathrm{E}$ & & & & \\
\hline Gross $\beta\left(\right.$ as $\left.\mathrm{Sr}^{90}-\mathrm{Y}^{90}\right)$ & 0.8 & & $12-28$ & \\
\hline Gross $\alpha$ as U equivalent $\mu \mathrm{g} / \mathrm{I}$ & 1.0 & & $12-28$ & \\
\hline Net extr. $\alpha$, U equivalent $\mu g / I$ & & & & \\
\hline Gross Y (as $\left.\operatorname{Cs}^{137}\right)$ & & & & \\
\hline Potassium ${ }^{40}$ & & & & \\
\hline Strontium $90 *$ & & & & \\
\hline Dissolved solids $\mathrm{mg} / 1$ & 17 & & & \\
\hline & & & & \\
\hline & & & & Corrected for \\
\hline Isotopic Analysis & & & Method & decay to: \\
\hline Barium-lanthanum 140 & & & & \\
\hline Cerium-praseodymium ${ }^{4} 4$ & & & & \\
\hline Cesium ${ }^{137}$ & & & & \\
\hline Chromium57 & & & & \\
\hline Cobalt & & & & \\
\hline Ruthenium-rhodium 106 & & & & \\
\hline $\begin{array}{lll}\text { Tritium } & (\mu \mathrm{c} / \mathrm{ml} & 2-9-66)\end{array}$ & $\overline{\mathrm{ND}}$ & & & \\
\hline Zinc 65 & & & & \\
\hline Zirconium-niobium 95 & & & & \\
\hline ND: Sought but not detected by & & & & \\
\hline method used. & & & & \\
\hline
\end{tabular}

Date sample received 12-14-65

Date data released $1-5-66$

Program classification

*Analyzed only when indicated by gross $\beta$ count.

Revised form $3-17-65$
Analyst T.Curran; P.K.Roscio; H.C.Claassen Date checked 1-5-66

Checked by V.r.Janzer

Checked from

Origina! Records

24

By

Date $2-22-66$

600048.780 


\section{U. S. DEPT. OF THE INTERIOR -- GEOLOGICAL SURVEY \\ Statement of Water Analysis}

Source We11 J3-2

Location Lumberton, Lamar Co., Mississippi

$\mathrm{NW} 1 / 4 \mathrm{SW} 1 / 4 \ldots 1 / 4 \mathrm{Sec} 3, \quad \mathrm{~T}-2 \mathrm{~N}, \mathrm{R}, 16 \mathrm{~W}$, Field/Office No.

Date Col. 10-26-65 Time_ 1115 Well Type Driven Use Dom; stock

Col. By USGS

Field detns: Tomp. $\left({ }^{\circ} \mathrm{F}\right)$

Sp. Cond. (umhos)

Appearance

F) 70

Clear
$\mathrm{pH}$ Eh

-

Depth (ft.) 30

Diam. (in.) 1-1/4 Cased to $27 \mathrm{ft}$.

Date drilled 1955

Water level ( $f$. 2 below 1.8.d., 1961

Discharge A few gpm (hand pump)

W. B. F.

Quaternary (alluvium, terrace deposits, etc.)

Owner W. E. Brown, Rt. 4, Lumberton, Miss.

\begin{tabular}{|c|c|c|}
\hline \multicolumn{3}{|c|}{ Chemical components } \\
\hline & ppm & epm \\
\hline Silica $\left(\mathrm{SiO}_{2}\right)$ & 20 & \\
\hline Aluminum (Á) & $<.01$ & \\
\hline Iron $\left(F_{e}\right)$ & $<.01$ & \\
\hline Manganese (Mn) & $<.02$ & \\
\hline Calcium (Ca) & 1.2 & 0.06 \\
\hline Magnesium (Mg) & .6 & .05 \\
\hline Strontium $(\mathrm{Sr})$ & .05 & $\cdots$ \\
\hline Sodium $(\mathrm{Na})$ & 4.7 & .20 \\
\hline Potassium (K) & .7 & .02 \\
\hline Lithium (Li) & $<.01$ & $-\infty$ \\
\hline Copper ( $\mathrm{Cu})$ & .04 & -- \\
\hline Zinc $(\mathrm{Zn})$ & .25 & -- \\
\hline
\end{tabular}

Physical characteristics and computed values

Dissolved Solids (ppm)

Res. on evap. at $180^{\circ} \mathrm{C}$

Calculated.

Suspended solids (ppm)

Hardness as $\mathrm{CaCO}_{3}$ (ppm)

Total

Non-carbonate

$\begin{array}{r}41 \\ \hline 37 \\ \hline 6 \\ \hline 0 \\ \hline 33 \\ \hline 6.2 \\ \hline 61 \\ \hline .9 \\ \hline\end{array}$

Cations (epm) $\quad 0.33$ Remarks:

Bicarbonate $\left(\mathrm{HCO}_{3}\right)$

Carbonate $\left(\mathrm{CO}_{3}\right)$

Sulfate $\left(\mathrm{SO}_{4}\right)$

Chloride $(\mathrm{Cl})$

Fluoride ( $F$ )

Nitrate $\left(\mathrm{NO}_{3}\right)$

Phosphate $\left(\mathrm{PO}_{4}\right)$

\begin{tabular}{cc}
14 & 0.23 \\
\hline 0 & .00 \\
\hline$<.1$ & -- \\
\hline 2.9 & .08 \\
\hline$<.1$ & -- \\
\hline .1 & .00 \\
\hline$<.01$ & -- \\
\hline
\end{tabular}

Specific conductance

(umhos at $25^{\circ} \mathrm{C}$ )

$\mathrm{pH}$

Color

Percent sodium

SAR

Anions (epm) $\quad 0.31$

Lab. No. 660445 Analysts

G. F. Scarbro 25

$Q W$ - IA

Original Records

Date Checked 1-14-66

By ....................................,

Date...3-3-66 
U. S. DEPT. OF THE INTERIOR--GEOLOGICAL SURVEY

Statement of Water Analysis - Radiochemical

Source Well J3-2

Location Lumberton, Lamar Co., Mississippi

$\mathrm{NW} 1 / \mathrm{SW}^{1 / 4} 1 / 4 \mathrm{Sec} 3, \mathrm{~T}_{2} \mathrm{~N}_{2} \mathrm{R} 16 \mathrm{~W}$. Field/Office No.

Date Col. 10-26-65 Time 1115

Col. By USGS

Fleld detns: Temp. (OF) 70

Sp. Cond. ( $\mu$ mhos )

Appearance

\section{clear}

\section{$\mathrm{h}$}

(2)

-

Well Type Driven

Depth $(\mathrm { ft } \longdiv { 3 0 }$

Diam. ( in) $1-1 / 4$ Date Drilled 1955

Water level (ft) 2 below 1.s.d., 1961

Discharge A few gpm (hand pump)

W.B.F. Quaternary (alluvium, terrace deposits, etc.)

Owner W. E. Brown,

Rt. 4, Lumberton, Miss.

Elements

$\mathrm{pc} / 1$

$\mu g / 1$

Uranium

Radium as $\operatorname{Ra}^{2 \mathrm{C}}$

Gross $\beta$ (as Sr80 - Yio)

Gross $\alpha$ as U equivalent $\mu \mathrm{g} / 1$

Net extr. $\alpha$, U equivalent $\mu \mathrm{g} / 1$

Gross $Y\left(a s \mathrm{Cs}^{137}\right)$

Potassiunto

Strontium 90 *

Dissolved solids $\mathrm{mg} / 1$

\begin{tabular}{l|l}
\hline & 37 \\
\hline & \\
\hline
\end{tabular}

Isotopic Analysis

Barium-lanthanum 140

Cerium-praseodymium ${ }^{4}{ }^{4}$

Cesium ${ }^{137}$

Chromium ${ }^{57}$

Cobalt ${ }^{60}$

Ruthenium_rhodium ${ }^{106}$

Tritium (uc/mi 2-9-66)

$\operatorname{Zinc}^{65}$

Zirconium-niobium ${ }^{95}$

ND: Sought but not detected by method used.

Date sample received 12-14-65

Date data released $1-17-66$

Program classification

$1-17-66$

*Analyzed only when indicated by gross $\beta$ count.

Revised form 3-17-65

$\mathrm{pc} / \mathrm{g}$

Date determined 


\section{U. S. DEPT. OF THE INTERIOR .. GEOLOGICAL SURVEY Statement of Water Analysis}

Source We11 J4-5

Locotion Lumberton, Lamar Co., Mississippi

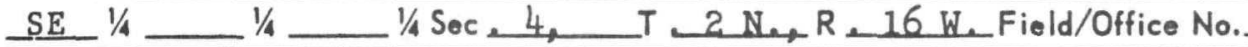

Date Col. 10-26-65_Time_1130___ Well Type Jetted Use Dom; stock

Col. By USGS

Field detns: Temp. $\left({ }^{\circ} \mathrm{F}\right)-74$

Sp. Cond. ( $\mu \mathrm{mhos}$ )

Appearance

Eh

H.

Depth (ft.) 25 Cased to $20 \mathrm{ft}$.

Diam. (in.) 2 Date drilled 1954?

Woter level (ft.) 16 below 1.8.d., 1954

Discharge Approx, $1 \mathrm{gpm}$

W. B. F. Quaternary (alluvium,

\begin{tabular}{cc} 
& terrace deposits, etc.) \\
\hline \multirow{2}{*}{ Owner } & Harry 0. Thompson, \\
\cline { 2 - 3 } & Rt. 4, Lumberton, Miss.
\end{tabular}

\begin{tabular}{|c|c|c|}
\hline \multicolumn{3}{|c|}{ Chemical components } \\
\hline & $\mathrm{ppm}$ & epm \\
\hline Silica $\left(\mathrm{SiO}_{2}\right)$ & 12 & \\
\hline Aluminum $(\mathrm{AI})$ & .01 & \\
\hline Iron $\left(\mathrm{Fe}_{\mathrm{e}}\right)$ & $<.01$ & \\
\hline Manganese $(M n)$ & .04 & \\
\hline Calcium (Ca) & 1.2 & 0.06 \\
\hline Magnesium (Mg) & 1.5 & .12 \\
\hline Strontium ( $\mathrm{Sr}$ ) & $<.02$ & - \\
\hline Sodium (Na) & 4.5 & .20 \\
\hline Potassium (K) & 1.5 & .04 \\
\hline Lithium (Li) & .01 & -- \\
\hline Copper ( $\mathrm{Cu}$ ) & .07 & -- \\
\hline $\operatorname{Zinc}(\mathrm{Zn})$ & .33 & -- \\
\hline
\end{tabular}

\section{Physical characteristics and computed values}

Dissolved Solids (ppm)

Res. on evap. at $180^{\circ} \mathrm{C}$

Calculated

Suspended solids (ppm)

Hardness as $\mathrm{CaCO}_{3}$ (ppm)

Total

Non-carbonate

\begin{tabular}{c}
41 \\
\hline 37 \\
\hline \\
\hline 9 \\
\hline 4 \\
\hline 57 \\
\hline 5.7 \\
\hline 48 \\
\hline .7 \\
\hline
\end{tabular}

Specific conductance

( $\mu$ mhos at $25^{\circ} \mathrm{C}$ )

$\mathrm{pH}$

Color

Percent sodium

SAR

Cations (epm) $\quad 0.42 \quad$ Remarks:

Bicarbonate $\left(\mathrm{HCO}_{3}\right)$

Carbonate $\left(\mathrm{CO}_{3}\right)$

Sulfate $\left(\mathrm{SO}_{4}\right)$

Chloride (Cl)

Fluoride ( $F$ )

Nitrate $\left(\mathrm{NO}_{3}\right)$

Phosphate $\left(\mathrm{PO}_{4}\right)$

\begin{tabular}{cc}
7 & 0.11 \\
\hline 0 & .00 \\
\hline$<.1$ & -- \\
\hline 5.7 & .16 \\
\hline$<.1$ & .00 \\
\hline 8.0 & .13 \\
\hline$<.01$ & -- \\
\hline & \\
\hline
\end{tabular}

Anions (epm)

0.40

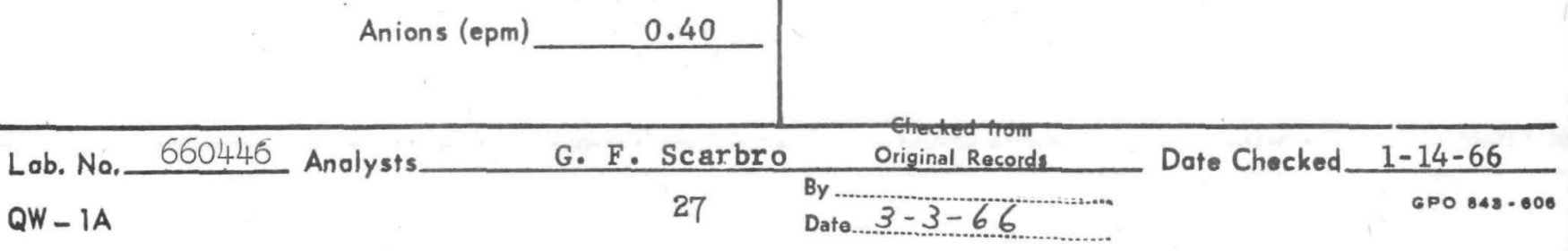


U. S. DEPT. OF THE INTERIOR--GEOLOGICAL SURVEY

Statement of Water Analysis - Radiochemical

Source We11 J4-5

Location Lumberton, Lamar Co., Mississippi

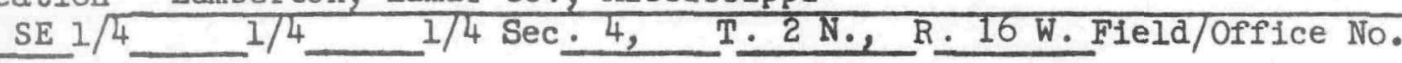

Date

Date Col. 10-26-65 Time 1130 Well Type Jetted Use Dom; stock

Col. By USGS Depth $(\mathrm { ft } \longdiv { 2 5 }$ Cased to $20 \mathrm{ft}$.

Fleld detas: Temp. (OF) 74 pH _ Diam. (In) Date Drilied 1954?

Sp. Cond. ( $\mu$ mhos) Eh - Water level (ft) 16 below 1.s.d., 1954

Appearance

\section{clear}

Discharge

Approx. $1 \mathrm{gpm}$

W.B.F. Quaternary (alluvium, terrace deposits, etc.)

Owner_Harry 0. Thompson, Rt. 4, Lumberton, Miss.

Elements

Uranium

Radium as Ra ${ }^{25 b}$

Gross $\beta\left(\right.$ a.s $\left.\mathrm{Sr}^{90}-\mathrm{Y}^{80}\right)$

Gross $\alpha$ as U equivalent $\mu \mathrm{g} / 1$

Net extr. $\alpha$, U equivalent $\mu \mathrm{g} / 1$

Gross $Y\left(\right.$ a.s $\left.\mathrm{Cs}^{137}\right)$

Potassium ${ }^{40}$

Strontium $90 *$

Dissolved solids $\mathrm{mg} / \mathrm{I}$

Isotopic Analysis

Barium-lanthanum ${ }^{20}$

Cerium-praseodymium ${ }^{166}$

Cesium ${ }^{13}$ ?

Chromium ${ }^{5}$ ?

Cobalt

Ruthenium_rhodium-06

Tritium

$\operatorname{Zinc}^{65}$

$(1 \mathrm{c} / \mathrm{ml} 2-9-66)$

Zirconium-n1obium ${ }^{85}$

ND: Sought but not detected by method used.

Date sample received 12-14-65

Date data released 2-11-66

Program classification

$\mu g / 1$

$1 / 1$
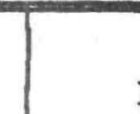

$\mathrm{pc} / 1$

$\mathrm{pc} / \mathrm{B}$

Date determined
Analyst P.K.Roscio; T.Curran; H.C.Claassen Date checked 2-11-66

Checked by
*Analyzed only when indlcated by gross $\beta$ count.

Revised form 3-17-65
28

Corrected for Method decay to:

Checked from

Original Records

By .

Date $2-22-66$ 


\section{U. S. DEPT. OF THE INTERIOR .- GEOLOGICAL SURVEY Statement of Water Analysis}

Source We11 J4-8

Location Lumberton, Lamar Co., Mississippi NW $1 / 4$ SW $1 / 4 \ldots 1 / 4$ Sec $4,4, T \cdot 2$ N., R. 16 W. Field/Office No.

Date Col. 10-26-65 Time 1145

Col. By USGS

Fiold detns: Temp. $\left({ }^{\circ} \mathrm{F}\right)$

Sp. Cond. ( $\mu$ mhos)

Appearance 1145 $\mathrm{pH}$ Eh

Clear

Well Type Drilled

Depth (ft.) 196

Diam. (in.) 2

Use Dom; stock

Water level (ft.) 60 below 1.s.d., 1959

Discharge $2 \mathrm{gpm}$ (estimated)

W. B. F. Pascagoula and Hattiesburg Fms., undiff.

Owner Ben F. Sones, Rt. 4, Lumberton, Miss.

\begin{tabular}{|c|c|c|}
\hline \multicolumn{3}{|c|}{ Chemical components } \\
\hline & ppm & epm \\
\hline Silica $\left(\mathrm{SiO}_{2}\right)$ & 54 & \\
\hline Aluminum (Al) & .01 & \\
\hline Iron (Fe) & $<.01$ & \\
\hline Manganese $(M n)$ & .90 & \\
\hline Calcium (Ca) & 27 & 1.35 \\
\hline Magnesium (Mg) & 6.6 & .54 \\
\hline Strontium (Sr) & .22 & -- \\
\hline Sodium (Na) & 21 & .91 \\
\hline Potassium (K) & 2.8 & .07 \\
\hline Lithium (Li) & .06 & -- \\
\hline Copper (Cu) & .02 & -- \\
\hline Zinc ( $\mathrm{Zn})$ & .38 & -- \\
\hline
\end{tabular}

Cations (epm) $\quad 2.89$

\section{Remarks:}

Physical characteristics and computed values

Dissolved Solids (ppm)

Res. on evap. af $180^{\circ} \mathrm{C}$

Calculated

Suspended solids (ppm)

Hardness as $\mathrm{CaCO}_{3}$ (ppm)

Total

Non-carbonate

Specific conductance

( $\mu$ mhos at $25^{\circ} \mathrm{C}$ )

$\mathrm{pH}$

Color

Percent sodium

SAR

212

202

$\begin{array}{r}212 \\ \hline 202 \\ \hline \\ \hline 95 \\ \hline 0\end{array}$

282

7.4

31

$\begin{array}{r}\frac{282}{7.4} \\ \hline 31 \\ \hline .9 \\ \hline\end{array}$

\section{Bicarbonate $\left(\mathrm{HCO}_{3}\right)$}

Carbonate $\left(\mathrm{CO}_{3}\right)$

Sulfate $\left(\mathrm{SO}_{4}\right)$

Chloride $(\mathrm{Cl})$

Fluoride $(F)$

Nitrate $\left(\mathrm{NO}_{3}\right)$

Phosphate $\left(\mathrm{PO}_{4}\right)$

\begin{tabular}{rr}
159 & 2.61 \\
\hline 0 & .00 \\
\hline 4.0 & .08 \\
\hline 8.5 & .24 \\
\hline .1 & .01 \\
\hline .1 & .00 \\
\hline$<.01$ & -- \\
\hline & \\
\hline
\end{tabular}

Anions (epm)

2.94

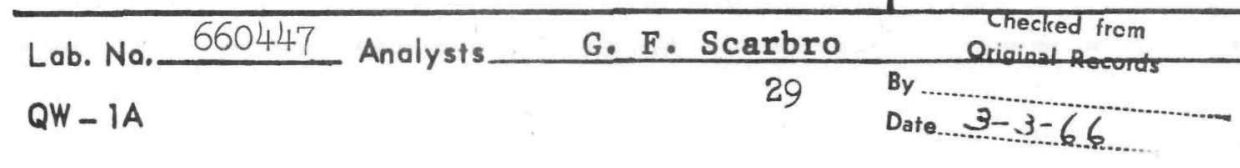


U. S. DEPI. OF THE INIERIOR-OGEOLOGICHE SURVEI

Statement of Water Analysis - Radiochemical

Source Well J4-8

Location Lumberton, Lamar Co., Mississippi
NW $1 / 4 \quad$ SW $1 / 4$
$1 / 4 \mathrm{Sec} 4, \quad$ T 2 N.
R 16 W. Field/Office No. 


\section{U. S. DEPT. OF THE INTERIOR -. GEOLOGICAL SURVEY} Statement of Water Analysis

Source We11 J10-1

Location Lumberton, Lamar Co., Mississippi SW $1 / 4$ SE $1 / 4 \ldots 1 / 4$ Sec. 10 , T. 2 N.2 R. $16 \mathrm{~W}$. Field/Office No.

Date Col. 10-26-65 Time 1515

Col. By USGS

Field detns: Temp. $\left({ }^{\circ} \mathrm{F}\right)$

Sp. Cond. (umhos)

Appearance

$\mathrm{pH}$

C1ear

Well Type Driven

Depth (ft.) 43

Diam. (in.) $1-1 / 4$

Water level (ft.)

Discharge

$22 / 38 p m, 3-27-63$

W. B. F.

Quaternary (alluvium, terrace deposits, etc.

Owner Toxie Speights, Rt. 4, Lumberton, Miss.

Use Dom; stock

\begin{tabular}{|c|c|c|}
\hline \multicolumn{3}{|c|}{ Chemical components } \\
\hline & $\mathrm{ppm}$ & epm \\
\hline Silica $\left(\mathrm{SiO}_{2}\right)$ & 7.8 & \\
\hline Aluminum (Al) & .02 & \\
\hline Iron $(\mathrm{Fe})$ & $<.01$ & \\
\hline Manganese (Mn) & $<.02$ & \\
\hline Calcium (Ca) & .2 & 0.01 \\
\hline Magnesium (Mg) & .4 & .03 \\
\hline Strontium ( $\mathrm{Sr}$ ) & $<.02$ & \\
\hline Sodium $(\mathrm{Na})$ & 2.8 & .12 \\
\hline Potassium (K) & .3 & .01 \\
\hline Lithium (Li) & $<.01$ & -- \\
\hline Copper (Cu) & .02 & -- \\
\hline Zinc (Zn) & .07 & -- \\
\hline
\end{tabular}

Physical characteristics and computed values

Dissolved Solids (ppm)

Res. on evap. at $180^{\circ} \mathrm{C}$

Calculated

uspended solids (ppm)

Hardness as $\mathrm{CaCO}_{3}$ (ppm)

Total

Non-carbonate

Specific conductance

( $\mu$ mhos at $25^{\circ} \mathrm{C}$ )

$\mathrm{pH}$

Color

Percent sodium

SAR

$\begin{array}{r}20 \\ \hline 18 \\ \hline\end{array}$

\begin{tabular}{c}
\hline 2 \\
\hline 0
\end{tabular}

6.3

$\begin{array}{r}17 \\ \hline 6.3 \\ \hline 71 \\ \hline .9\end{array}$

\section{Remarks:}

Bicarbonate $\left(\mathrm{HCO}_{3}\right)$

Carbonate $\left(\mathrm{CO}_{3}\right)$

Sulfate $\left(\mathrm{SO}_{4}\right)$

Chloride (Cl)

Fluoride (F)

Nitrate $\left(\mathrm{NO}_{3}\right)$

Phosphate $\left(\mathrm{PO}_{4}\right)$

\begin{tabular}{cc}
4 & 0.07 \\
\hline 0 & .00 \\
\hline 1.0 & .02 \\
\hline 2.6 & .07 \\
\hline$<.1$ & -- \\
\hline .7 & .01 \\
\hline$<.01$ & -- \\
\hline
\end{tabular}

Anions (epm)

0.17
Lab. No. 660448 Analysts_ G. F. Scarbro

$Q W-1 A$ -inecked Trom

Original Recoeds

By.

Date $3-3-66$
Date Checked 1-14-66

GPO 843.008 
U. S. DEPT. OF THE INTERIOR--GEOLOGICAL SURVEY

Statement of Water Analysis - Radiochemical

Source

Well J10-1

Location Lumberton, Lamar Co., Mississippi

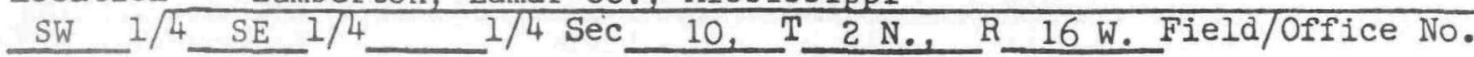

Date Col.10-26-65 Time 1515 $\quad$ Well Type Driven Use Dom; stock

Col. By USGS

Field detrs: Temp. ( ${ }^{\circ} \mathrm{F}$ $\mathrm{Sp}$. Cond. ( $\mu$ mhos)

Appearance

$67 \quad \mathrm{pH}$ Eh Clear
Depth ( $1 t)-43$

Diam. (in) $1-1 / 4$

Water level (ft)

Discharge $2 2 \longdiv { 3 \mathrm { gpm } , 3 - 2 7 - 6 5 }$

W.B.F. Quaternary (alluvium, terrace deposits, etc.

Toxie Speights.

Rt. 4, Lumberton, Miss.

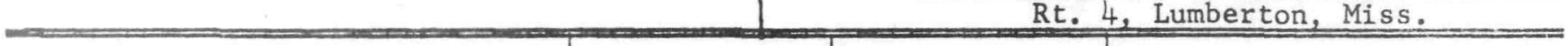

Elements

Urantum

Radium as $\mathrm{Re}^{\mathrm{C} 2 \mathrm{~b}}$

Grogs $\beta\left(\right.$ as $\left.\mathrm{Sr}^{90}-\mathrm{Y}^{80}\right)$

Gross $\alpha$ as U equivalent $\mu \mathrm{g} / 1$

Net extr. $\alpha$, U equivalent $\mu \mathrm{g} / 1$

Gross $Y$ (as $\left.\mathrm{Cs}^{137}\right)$

Potassium ${ }^{40}$

Strontium90*

Dissolved solids mg/1

$\mu \mathrm{B} / 1$

Isotopic Analysis

Barium-lanthanum 140

Cerium-praseodymium ${ }^{146}$

Cesium 137

Chromium 5 7

Cobaltoo

Ruthenium-rhodium ${ }^{106}$

Tritium (uc/ml 2-9-66)

Zinc $^{65}$

Zirconium-niobium ${ }^{95}$

ND: Sought but not detected by method used,

Date sample received 12-14-65

Da.te do.ta released $1-5-66$

Program classification

Date determined

$\mathrm{pc} / 1$

pc/s

$\frac{12-28-65}{12-28-65}$

*Analyzed only when indicated by gross $\beta$ count.

Revised form 3-17-65

Analyst T,Curran; E.Villasana; H.C.Claasse Date checked 1-5-66

Checked by V.J. Janzer

Method

Corrected for decay to: 


\section{U. S. DEPT. OF THE INTEPIOR .- GEOLOGICAL SURVEY Statement of Water Analysis}

Source We11 J12-1

Location Lumberton, Lamar Co., Mississippi

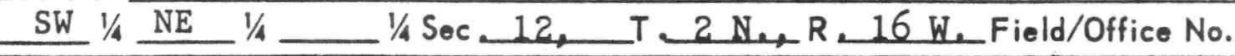

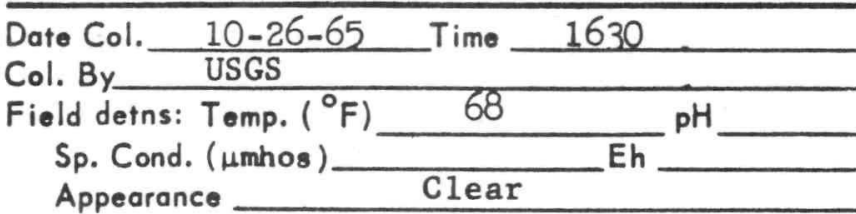

\begin{tabular}{|c|c|c|}
\hline \multicolumn{3}{|c|}{ Chemical components } \\
\hline & ppm & epm \\
\hline Silica $\left(\mathrm{SiO}_{2}\right)$ & 7.6 & \\
\hline Aluminum (Al) & .01 & \\
\hline Iron (Fe) & $<.01$ & \\
\hline Manganese (Mn) & .03 & \\
\hline Calcium (Ca) & .5 & 0.02 \\
\hline Magnesium (Mg) & .8 & .07 \\
\hline Strontium (Sr) & $<.02$ & $-\div$ \\
\hline Sodium $(\mathrm{Na})$ & 3.3 & .14 \\
\hline Potassium (K) & .7 & .02 \\
\hline Lithium (Li) & .01 & -- \\
\hline Copper ( $\mathrm{Cu})$ & .02 & -- \\
\hline Zinc $(\mathrm{Zn})$ & .21 & \\
\hline
\end{tabular}

\begin{tabular}{|c|c|c|}
\hline We & Bored & e Bom; stock \\
\hline Dept & 48 & $47 \mathrm{ft}$. \\
\hline (in.) & 8 & Date drilled 1943 \\
\hline
\end{tabular}

Water level (ft.) 31.19 below 1.s.d., 7-22-65

Discharge Bucket well

W. B. F. Quaternary (alluvium,
- terrace deposits, etc.)
Owner Fred Dobson,

\begin{tabular}{c|l} 
& \\
\hline $\begin{array}{c}\text { Chemical components } \\
\text { ppm }\end{array}$ & \\
\hline
\end{tabular}

\section{Physical characteristics and computed values}

Dissolved Solids (ppm)

Res. on evap. of $180^{\circ} \mathrm{C}$

Calculated

Suspended solids (ppm)

Hardness as $\mathrm{CaCO}_{3}(\mathrm{ppm})$

Total

Non-carbonate

Specific conductance

(umhos at $25^{\circ} \mathrm{C}$ )

$\mathrm{pH}$

Color

Percent sodium

SAR

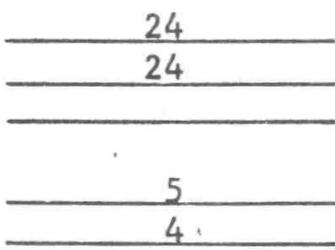

36

5.3

56

Cations (epm) $\quad 0.25 \quad$ Remarks:

Bicarbonate $\left(\mathrm{HCO}_{3}\right)$

Carbonate $\left(\mathrm{CO}_{3}\right)$

Sulfate $\left(\mathrm{SO}_{4}\right)$

Chloride (Cl)

Fluoride (F)

Nitrate $\left(\mathrm{NO}_{3}\right)$

Phosphate $\left(\mathrm{PO}_{4}\right)$

\begin{tabular}{cc}
1 & 0.02 \\
\hline 0 & .00 \\
\hline$<.1$ & -- \\
\hline 5.8 & .16 \\
\hline$<.1$ & -- \\
\hline 4.8 & .08 \\
\hline$<.01$ & -- \\
\hline & \\
\hline & \\
\hline
\end{tabular}

Anions (epm)

0.26

Lab. No. 660449 Analysts

$Q W-1 A$
G. F. Scarbro

33

Checked Trom

Oriainal Racards

By ....................-

Date...3-3-66
Date Checked 1-14-66

GPO $043 \cdot 006$ 
U. S. DEPT. OF THE INTERIOR--GEOLOGICAL SURVEY

Statement of Water Analysis - Radiochemical

Source We11 J12-1

Location Lumberton, Lamar Co., Mississippi
SW
$1 / 4$ NE $1 / 4$
$1 / 4 \mathrm{Sec} 12, \mathrm{~T} 2 \mathrm{~N}$.
R16 W. Field/Office No.

Date Col. 10-26-65 Time 1630

Col. By USGS

Field detns: Temp. ( $\left.{ }^{\circ} \mathrm{F}\right)$

Sp. Cond. ( $\mu$ mhos)

Appearance

$\frac{68 \mathrm{EH}}{\mathrm{ph}}$

clear

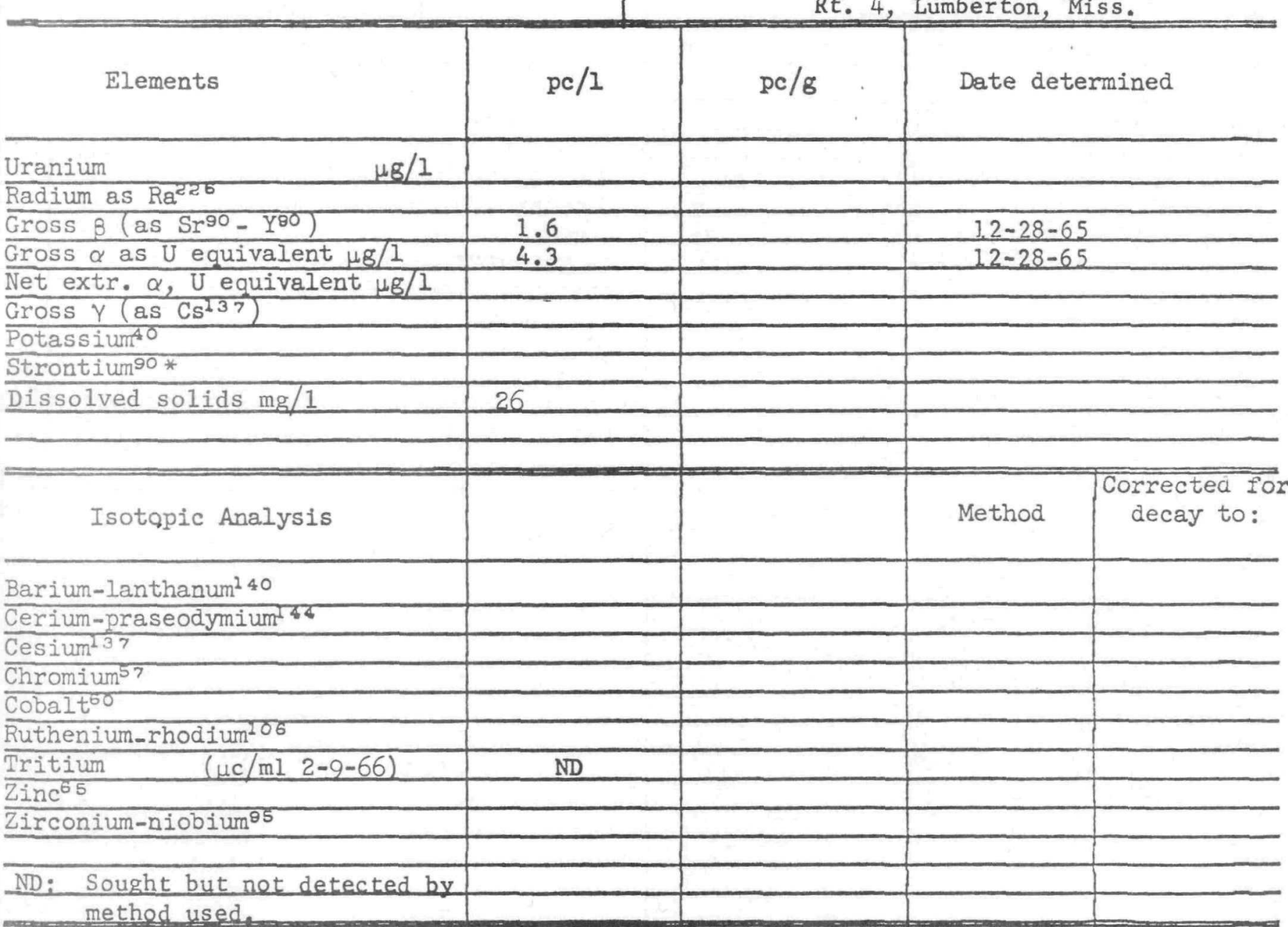

Date sample received 12-14-65

Date data released $1-5-66$

Program classification

*Analyzed only when indicated by gross $\beta$ count.

Revised form 3-17-65
Well Type Bored

Depth ( $\mathrm { ft } \longdiv { 4 8 }$

Diam. (in) 8

Water level (ft) 31.19 below 1.s.d., 7-22-65

Discharge Bucket well

W.B.F. Quaternary (alluvium, terrace deposits, etc.)

Owner Fred Dobson, Rt. 4, Lumberton, Miss.

Use Dom: stock Cased to $47 \mathrm{ft}$. Date Drilled 1943
AnolystT.Curran; E.Villasana; H.C.Claasse Date checked $1-5-66$

Checked by Vhr.Ianzer

$12-28-65$ $12-28-65$ 
Source We11 J12-2

Location Lumberton, Lamar Co., Mississippi NW $1 / 4$ NE $1 / 4 \ldots 1 / 4$ Sec 12 , T 2 N $2, R, 16$ W. Field/Office No.

Date Col. 10-26-65 Time 1640

Col. By USGS

Fiold detns: Temp. $\left({ }^{\circ} \mathrm{F}\right) \quad 69$

Sp. Cond. (umhos)

Appearance

\section{Clear}

$\mathrm{pH}$

Eh

$\longrightarrow$

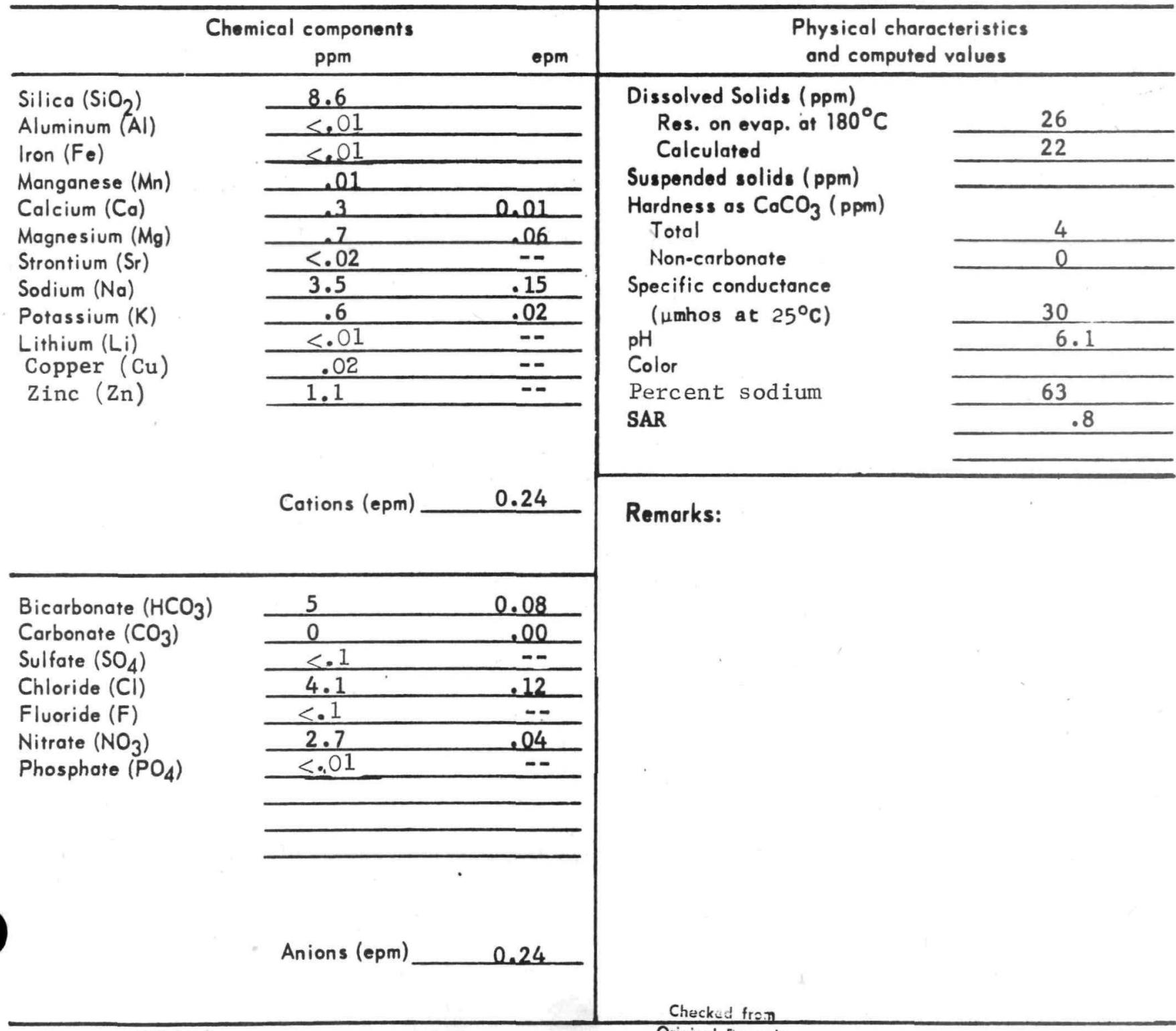


U. S. DEPT. OF THE INTERIOR--GEOLOGICAI SURVEY

Statement of Water Analysis - Radiochemical

Source

We11 J12-2

Location Lumberton, Lamar Co., Mississippi $\mathrm{NW} 1 / \mathrm{NE}^{1 / 4} 1 / 4 \mathrm{Sec} 12, \mathrm{~T}^{\mathrm{T}} 2 \mathrm{~N}, \mathrm{~N}^{\mathrm{R}} 16 \mathrm{~W}$. Field/Office No.

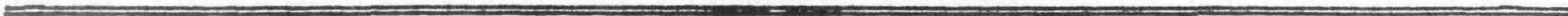
Date Col. 10-26-65 Time 1640 Well Type Drilled Use Dom; stock

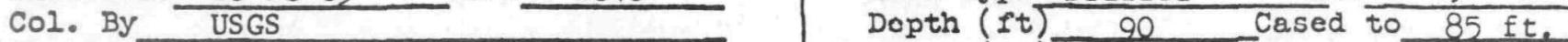
Field detas: Temp. ( $\left.{ }^{\mathrm{F}}\right) 69 \mathrm{pH} \quad$ Diam. (in) $\frac{2}{2}$ Date Drilled 1951 Sp. Cond. ( $\mu$ mhos) Appearance clear Water level (ft) Unknown

Discharge $8 \mathrm{gpm}, 8-18-64$ (measured) W.B.F. Quaternary (alluvium, terrace deposits, etc.) Owner Ross Powell

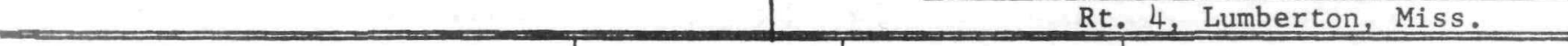

\begin{tabular}{|c|c|c|c|}
\hline Elements & $\mathrm{pc} / \mathrm{l}$ & $\mathrm{pc} / \mathrm{g}$ & Date determined \\
\hline Uranium & & - & \\
\hline \multicolumn{4}{|l|}{ Radium as $\mathrm{Ra}^{22 \mathrm{~b}}$} \\
\hline Gross $\beta\left(\right.$ as $\left.\mathrm{Sr}^{90}-\mathrm{Y}^{00}\right)$ & 1.4 & & $12-28-65$ \\
\hline Gross $\alpha$ as U equivalent $\mu g / 1$ & 2.8 & & $12-28-65$ \\
\hline \multicolumn{4}{|l|}{ Net extr. $\alpha, \mathrm{U}$ equivalent $\mu g / 1$} \\
\hline \multicolumn{4}{|l|}{ Gross Y (as $\left.\mathrm{Cs}^{137}\right)$} \\
\hline \multicolumn{4}{|l|}{ Potassium ${ }^{40}$} \\
\hline \multicolumn{4}{|l|}{ Strontium $90 *$} \\
\hline Dissolved solids mg/l & 24 & & \\
\hline & & & \\
\hline
\end{tabular}

Isotopic Analysis

Barium-lanthanum 10

Cerium-praseodymium ${ }^{46}$

Cesium ${ }^{13} 7$

Chromium 57

Cobalt ${ }^{60}$

Ruthenium-rhodium ${ }^{106}$

Tritium

$\mathrm{Zinc}^{65}$

$(\mu c / m 12-9-66)$

Zirconium-niobium ${ }^{95}$

ND: Sought but not detected by method used

Date sample received 12-14-65

Date data released $1-5-66$

Program classification

Analyst T.Curran: E.Villasana: H.C.Claas Date checked 1-5-66

*Analyzed only when indicated by gross $\beta$ count. 


\section{U. S. DEPT. OF THE INTERIOR -- GEOLOGICAL SURVEY Statement of Water Analysis}

Source We11 J16-7

Location Lumberton, Lamar Co., Mississippi $\mathrm{SE} 1 / \mathrm{NE} 1 / 4 \ldots 1 / 4 \mathrm{Sec} \cdot 16, T-2 \mathrm{~N}, \mathrm{R} \cdot 16 \mathrm{~W}$. Field/Office No.

Date Col._10-26-65_Time 1235_ Well Type Bored Use_ Dom; stock

Col. By USGS

Field deens: Temp. ( $\left.{ }^{\circ} \mathrm{F}\right) 68$

Sp. Cond. ( $\mu \mathrm{mhos}$ )

Appearance

\section{Clear}

$\mathrm{pH}$

Eh

Depth (ft.) 65.80

Diam. (in.) 6 Cosed to $67 \mathrm{ft}$. (est.)

Woter lovel (ft.) 48.64 below 1.s.d., 7-22-65

Dischorge Bucket wel1

W. B. F. Quaternary (alluvium, - terrace deposits, etc.)

Owner Wylie Smith, Rt. 4, Lumberton, Miss.

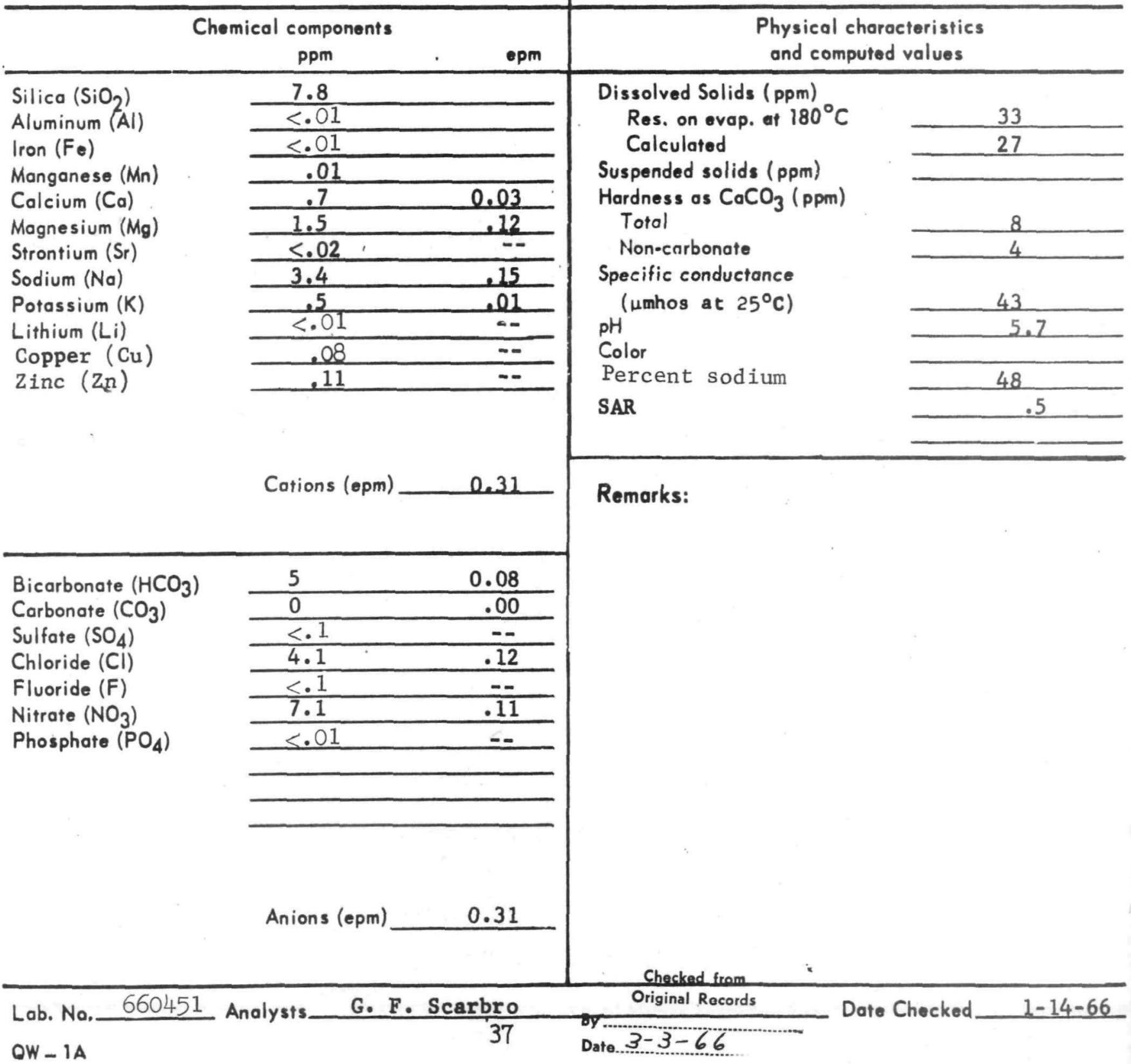


Lab. No.

U. S. DEPT. OF THE INTERIOR--GEOLOGICAL SURVEY

Statement of Water Analysis - Radiochemital

Source

Well J16-7

Location Lumberton, Lamar Co., Mississippi

$\mathrm{SE} 1 / 4 \mathrm{NE} 1 / 4 \ldots 1 / 4 \mathrm{Sec}-16,{ }^{\mathrm{T}} 2 \mathrm{~N}, \mathrm{R}^{\mathrm{R}} 16 \mathrm{~W}$, Field/Office No.

Date Col. 10-26-65 Time 1235 Well Type Bored Use Dom; stock

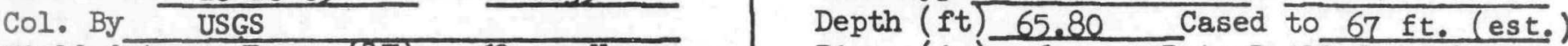

Field detns: Temp. ( $\left.{ }^{\circ} \mathrm{F}\right) 68$ pH__ Diam. (in $\frac{6}{6}$ Date Drilled 1949

Sp. Cond. ( $\mu$ mhos)

Appearance

Water level (ft) 48.64 below 1.s.d., 7-22-65

Discharge Bucket well

W.B.F. Quaternary (alluvium, terrace deposits, etc.)

Owner Wylie Smith, Rt. 4, Lumberton, Miss .

\begin{tabular}{|c|c|c|c|c|}
\hline Elements & $\mathrm{pc} / 1$ & $\mathrm{pc} / \mathrm{g}$ & \multicolumn{2}{|c|}{ Date determined } \\
\hline \multirow{2}{*}{\multicolumn{5}{|c|}{$\begin{array}{l}\text { Uranium } \mu g / 1 \\
\text { Radium as Ra }\end{array}$}} \\
\hline & & & & \\
\hline Gross $\beta\left(\operatorname{as~Sr} 90-Y^{90}\right)$ & 1.3 & & \multicolumn{2}{|c|}{$1-7-66$} \\
\hline Gross $\alpha$ as U equivalent $\mu \mathrm{g} / 1$ & .8 & & \multicolumn{2}{|c|}{$1-7-66$} \\
\hline \multicolumn{5}{|l|}{ Net extr. $\alpha, U$ equivalent $\mu \delta / I$} \\
\hline \multicolumn{5}{|l|}{ Gross Y (as $\left.\mathrm{Cs}^{137}\right)$} \\
\hline \multicolumn{5}{|l|}{$\frac{\text { Potassium }}{\text { Strontium }}$} \\
\hline Strontium $90 *$ & & & & \\
\hline \multirow{2}{*}{\multicolumn{5}{|c|}{ Dissolved solids mg/1 }} \\
\hline & & & & \\
\hline \multicolumn{5}{|l|}{ 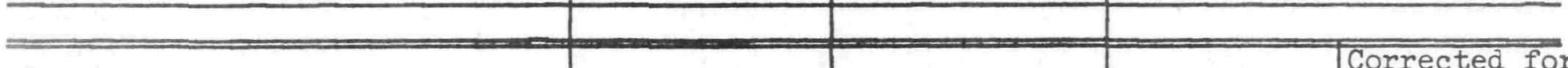 } \\
\hline Isotopic Analysis & & & Method & $\begin{array}{l}\text { Corrected for } \\
\text { decay to: }\end{array}$ \\
\hline \multicolumn{5}{|l|}{ Barium-lanthanum 140} \\
\hline \multicolumn{5}{|l|}{ Cerium-praseodymium ${ }^{146}$} \\
\hline \multicolumn{5}{|l|}{ Cesium ${ }^{137}$} \\
\hline \multicolumn{5}{|l|}{ Chromium 57} \\
\hline \multicolumn{5}{|l|}{ Cobalt } \\
\hline \multicolumn{5}{|l|}{ Ruthenium_rhodium ${ }^{106}$} \\
\hline$(u c / m 12-9-66)$ & ND & & & \\
\hline \multicolumn{5}{|l|}{ Zinc 65} \\
\hline \multicolumn{5}{|l|}{ Zirconium-niobium ${ }^{95}$} \\
\hline \multirow{2}{*}{\multicolumn{5}{|c|}{ ND: Sought but not detected by }} \\
\hline & & & & \\
\hline
\end{tabular}

Date sample received

$12-14-65$

Date data released $1-17-66$

Program classification

*Analyzed only when indicated by gross $\beta$ count.

Revised form 3-17-65
Analyst T.Curran; E.Willasana: H.C.Claasse Date checked 1-17-66 Checked by V. J. Janzer

\section{Checked from}

Original Records

By.

Date $2-2<-66$ 


\section{U. S. DEPT. OF THE INTERIOR -. GEOLOGICAL SURVEY}

Statement of Water Analysis

Source We11 J16-10

Location Lumberton, Lamar Co., Mississippi

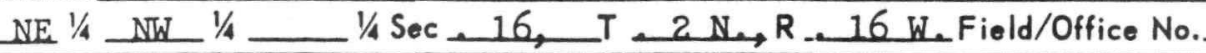

Date Col. 10-26-65_Time_1200_ Well Type Bored_Use Dom; stoak

Col. By USGS

Fiold detns: Tomp. $\left({ }^{\circ} \mathrm{F}\right)-66 \quad \mathrm{pH}$

Sp. Cond. (umhos)

Appearance
Depth (ft.) 39

Diam. (in.)

Water level ( $\mathrm{ft}$.) 15 below 1.s.d., 1951

Discharge A few gpm (estimated)

W. B. F. Quaternary (alluvium, terrace, deposits, etc.)

Owner Archie Sistrunk, Rt. 4, Lumberton, Miss.

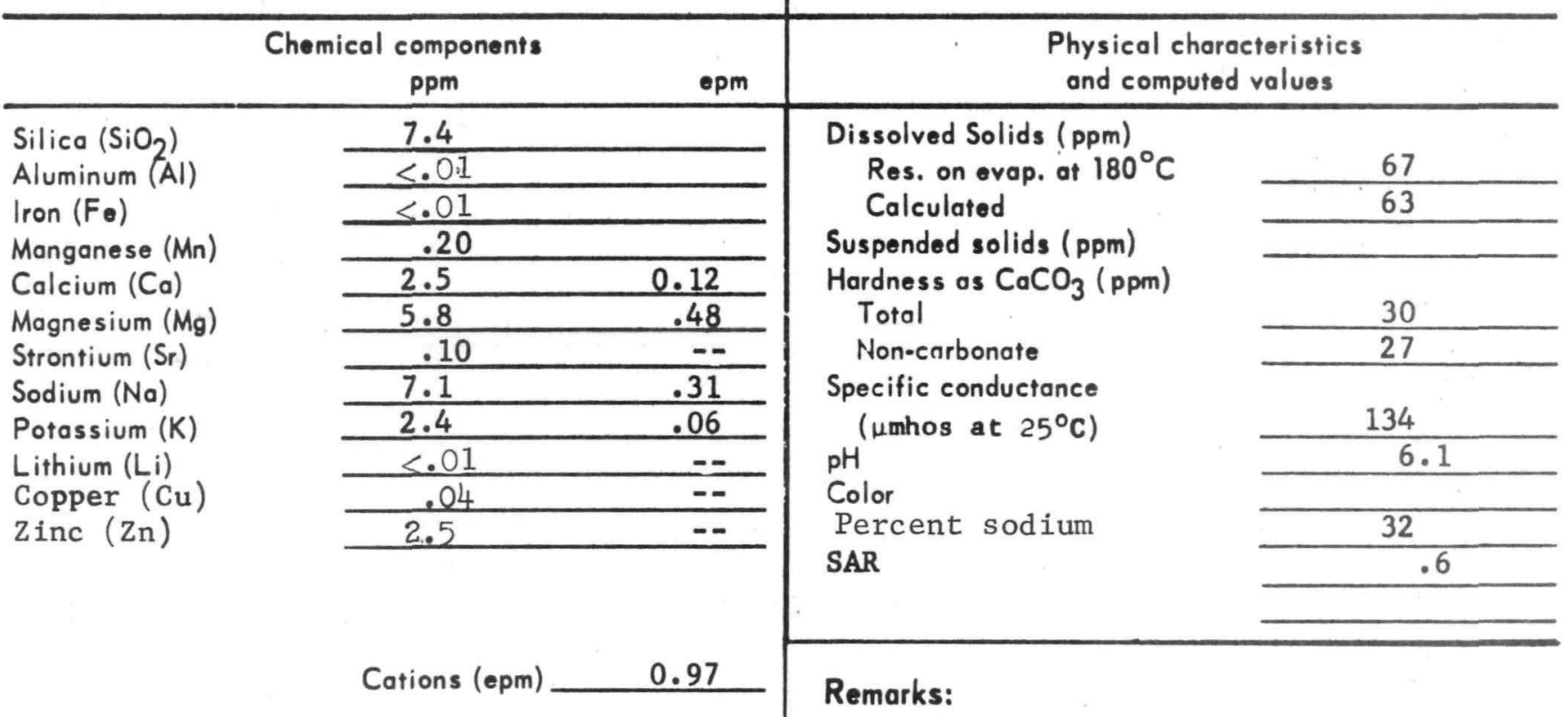

Bicarbonate $\left(\mathrm{HCO}_{3}\right)$

Carbonate $\left(\mathrm{CO}_{3}\right)$

Sulfate $\left(\mathrm{SO}_{4}\right)$

Chloride $(\mathrm{Cl})$

Fluoride (F)

Nitrate $\left(\mathrm{NO}_{3}\right)$

Phosphate $\left(\mathrm{PO}_{4}\right)$

\begin{tabular}{cc}
4 & 0.07 \\
\hline 0 & .00 \\
\hline 1.0 & .02 \\
\hline 24 & .68 \\
\hline$<.01$ & -- \\
\hline 11 & .18 \\
\hline$<.01$ & -- \\
\hline
\end{tabular}


U. S. DEPT. OF THE INTERIOR--GEOLOGICAL SURVEY

Statement of Water Analysis - Radiochemical

Source Well J16-10

Location Lumberton. Lamar Coe, Mississippi

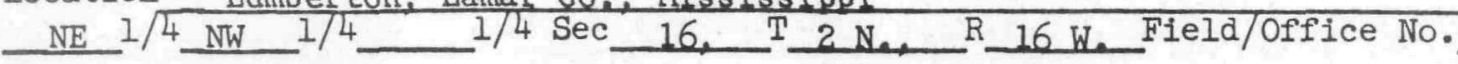

ב-1000

Date Col. 10-26-65 Time 1200 Well Type Bored Use Dom; stock

Col. By USGS Depth ( ft 39 Cased to $39 \mathrm{ft}$.

Field detns: Temp. (of) 66 pH Diam. (in) 6 Date Drilled 1951

Sp. Cond. ( $\mu$ mhos) Eh - Water level (ft) 15 below 1.s.d., 1951

Appearance

Clear

Discharge A few gpm (estimated)

W.B.F. Quaternary (alluvium,

terrace deposits, etc.)

Owner Archie Sistrunk,

\begin{tabular}{|c|c|c|c|c|}
\hline Elements & $\mathrm{pc} / \mathrm{l}$ & $\mathrm{pc} / \mathrm{g}$ & \multicolumn{2}{|c|}{ Date determined } \\
\hline \multicolumn{5}{|l|}{ Uranium $\mu g / 1$} \\
\hline Radium as $\mathrm{Ra}^{2 \mathrm{C}}$ & & & & \\
\hline Gross $\beta\left(\right.$ as $\left.\mathrm{Sr}^{90}-\mathrm{Y}^{90}\right)$ & 10 & & \multicolumn{2}{|c|}{$1-7-66$} \\
\hline Gross $\alpha$ as U equivalent $\mu g / 1$ & 6.3 & & \multicolumn{2}{|c|}{$1-7-66$} \\
\hline \multicolumn{5}{|l|}{ Net extr. $\alpha$, U equivalent $\mu g / 1$} \\
\hline \multicolumn{5}{|l|}{ Gross $Y\left(\right.$ as $\left.\operatorname{Cs}^{137}\right)$} \\
\hline \multicolumn{5}{|l|}{ Potassium 40} \\
\hline \multicolumn{5}{|l|}{ Strontium $90 *$} \\
\hline \multirow{2}{*}{\multicolumn{5}{|c|}{ Dissolved solids $\mathrm{mg} / 1$}} \\
\hline & & & & \\
\hline \\
\hline Isotopic Analysis & & & Method & $\begin{array}{l}\text { Corrected for } \\
\text { decay to: }\end{array}$ \\
\hline \multicolumn{5}{|l|}{ Barium-lanthanum 140} \\
\hline \multicolumn{5}{|l|}{ Cerium-praseodymium ${ }^{14}$} \\
\hline \multicolumn{5}{|l|}{ Cesium } \\
\hline \multicolumn{5}{|l|}{ Chromium ${ }^{\text {7 }}$} \\
\hline \multicolumn{5}{|l|}{ Cobalt } \\
\hline \multicolumn{5}{|l|}{ Ruthenium_rhodium ${ }^{106}$} \\
\hline$(\mu \mathrm{c} / \mathrm{ml} 2-9-66)$ & ND & & & \\
\hline \multicolumn{5}{|l|}{$\operatorname{Zinc}^{65}$} \\
\hline \multicolumn{5}{|l|}{ Zirconium-niobium ${ }^{95}$} \\
\hline & & & & \\
\hline ND: Sought but not detected by & & & & \\
\hline method used. & & & & \\
\hline
\end{tabular}

Date sample received 12-14-65

Date data released $1-17-66$

Program classification

*Analyzed only when indicated by gross $\beta$ count.

Revised form 3-17-65
Analyst T.Curran: P.K.Roscio: H.C.Claassen Date checked 1-17-66

Checked by V.J. Janzer

$40 \quad \begin{gathered}\text { Checked from } \\ \text { Original Rocords }\end{gathered}$




\section{U. S. DEPT. OF THE INTERIOR -. GEOLOGICAL SURVEY \\ Statement of Water Analysis}

Source We11 J16-11

Location Lumberton, Lamar Co., Mississippi

SE $1 / 4$ SW $1 / 4$ _ $1 / 4$ Sec. 16, T. 2 Ne, R. 16 W. Field/Office No.

Date Col. 10-26-65 Time 1225

Col. By USGS

Field derns: Temp. $\left({ }^{\circ} \mathrm{F}\right)$

Sp. Cond. (umhos)

Appearance
68

Eh

$\mathrm{pH}$

Clear
Well Type Drilled

Depth ( ft.) 100

Diam. (in.)

2 Use Dom; stock Cosed to $96 \mathrm{ft}$. Date drilled 1952

Water level ( $\mathrm{ft}$.) 80 below 1.s.d., 1952

Discharge A few gpm (estimated)

W. B. F. Quaternary (alluvium, terrace deposits, etc.)

Owner Mark Lowe, Rt. 4, Lumberton, Miss.

\section{Chemical components}

Silica $\left(\mathrm{SiO}_{2}\right)$

Aluminum (Al)

Iron $\left(F_{0}\right)$

Manganese $(\mathrm{Mn})$

Calcium ( $\mathrm{Ca}$ )

Magnesium (Mg)

Strontium ( $\mathrm{Sr}$ )

Sodium $(\mathrm{Na})$

Potossium (K) -

Lithium ( $\mathrm{Li}$ )

Copper (Cu)

Zinc $(\mathrm{Zn})$

\begin{tabular}{|c|c|}
\hline ppm & epm \\
\hline \multicolumn{2}{|l|}{9.0} \\
\hline \multicolumn{2}{|l|}{.02} \\
\hline$<.01$ & \\
\hline \multicolumn{2}{|l|}{.01} \\
\hline 2.7 & 0.13 \\
\hline 1.0 & .08 \\
\hline .08 & -- \\
\hline 3.7 & .16 \\
\hline .6 & .02 \\
\hline$<.01$ & -- \\
\hline $.05^{\circ}$ & - \\
\hline .08 & -- \\
\hline
\end{tabular}

Cations (epm)

0.39

Bicarbonate $\left(\mathrm{HCO}_{3}\right)$

Carbonate $\left(\mathrm{CO}_{3}\right)$

Sulfate $\left(\mathrm{SO}_{4}\right)$

Chloride (Cl)

Fluoride (F)

Nitrate $\left(\mathrm{NO}_{3}\right)$

Phosphate $\left(\mathrm{PO}_{4}\right)$
Physical characteristics

and computed values

Dissolved Solids (ppm)

Res. on evap. at $180^{\circ} \mathrm{C}$

Calculated

Suspended solids (ppm)

Hardness as $\mathrm{CaCO}_{3}$ (ppm)

Total

Non-carbonate

Specific conductance

( $\mu$ mhos at $25^{\circ} \mathrm{C}$ )

$\mathrm{pH}$

Color

Percent sodium

SAR

$\begin{array}{r}34 \\ \hline 32 \\ \hline \\ \hline 11 \\ \hline 3\end{array}$

51

6.1

41

\section{Remarks:}

Lab. No. $\frac{660453}{\cdot}$ Analysts $\quad$ G. F. Scarbro $\frac{\text { Original Records }}{41}$ Date Checked $1-14-66$


U. S. DEPT. OF THE INTERIOR--GEOLOGICAL SURVEY

Statement of Water Analysis - Radiochemical

Source We11 J16-11

Location Lumberton, Lamar Co., Mississippi $\mathrm{SE} 1 / 4 \mathrm{SW} 1 / 4 \quad 1 / 4 \mathrm{Sec} 16, \mathrm{~T}_{2} \mathrm{~N}_{1} \mathrm{R}_{16} \mathrm{~W}$. Field/Office No.

Date Col. 10-26-65 Time_1225 $\quad$ Well Type Drilled Use Dom; stock

Field detns: Temp. (oF)

Sp. Cond. ( $\mu$ mhos)

Appearance Clear
Col. By USGS

$68 \mathrm{Eh}^{\mathrm{pH}}$

Depth ( ft 100

Diam. (in) $\frac{1}{2}$ Date Drilled 1952

Water level (ft) 80 below 1.s.d. 1952

Discharge A few gpm (estimated)

W.B.F. Quaternary (alluvium, terrace deposits, etc.)

Owner Mark Lowe, Rt. 4, Lumberton, Mississippi

\begin{tabular}{|c|c|c|c|c|}
\hline Elements & \multirow[t]{2}{*}{$\mathrm{pc} / 1$} & \multirow[t]{2}{*}{$\mathrm{pc} / \mathrm{g}$} & \multicolumn{2}{|c|}{ Date determined } \\
\hline \multirow{2}{*}{\multicolumn{5}{|c|}{$\begin{array}{l}\text { Uranium } \\
\text { Radium as } \mathrm{Ra}^{2 \mathrm{~L}} \quad \mu \mathrm{g} / \mathrm{l}\end{array}$}} \\
\hline & & & & \\
\hline Gross $\beta\left(\right.$ as $\left.\mathrm{Sr}^{90}-\mathrm{Y}^{90}\right)$ & 0.8 & & \multicolumn{2}{|c|}{$1-7-66$} \\
\hline Gross $\alpha$ as U equivalent $\mu \mathrm{g} / 1$ & 1.3 & & \multicolumn{2}{|c|}{$1-7-66$} \\
\hline \multicolumn{5}{|l|}{ Net extr. $\alpha, U$ equivalent $\mu g / 1$} \\
\hline \multicolumn{5}{|l|}{ Gross $y\left(\right.$ as $\left.\mathrm{Cs}^{137}\right)$} \\
\hline \multicolumn{5}{|l|}{ Potassium } \\
\hline \multicolumn{5}{|l|}{ Strontium90 * } \\
\hline \multicolumn{5}{|l|}{ Dissolved solids $\mathrm{mg} / \mathrm{l}$} \\
\hline \multicolumn{5}{|l|}{ 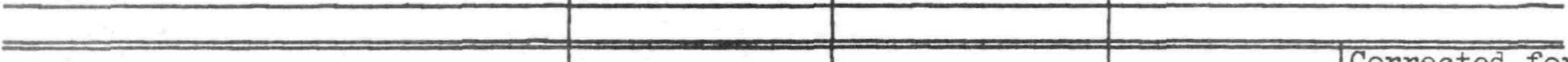 } \\
\hline Isotopic Analysis & & & Method & $\begin{array}{l}\text { Corrected for } \\
\text { decay to: }\end{array}$ \\
\hline \multicolumn{5}{|l|}{ Barium-lanthanum 140} \\
\hline \multicolumn{5}{|l|}{ Cerium-praseodymium ${ }^{46}$} \\
\hline \multicolumn{5}{|l|}{ Cesium ${ }^{137}$} \\
\hline Chromium ${ }^{7}$ & & & & 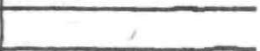 \\
\hline \multicolumn{5}{|l|}{ Cobalt 60} \\
\hline \multicolumn{5}{|l|}{ Ruthenium_rhodium ${ }^{106}$} \\
\hline Tritium $\quad(\mu \mathrm{c} / \mathrm{ml} 2-9-66)$ & ND & & & \\
\hline \multicolumn{5}{|l|}{ Zinc 65} \\
\hline \multicolumn{5}{|l|}{ Zirconium-niobium } \\
\hline & & & & \\
\hline ND: Sought but not detected by & & & & \\
\hline method used. & & & & \\
\hline
\end{tabular}

Date sample received 12-14-65

Date data released $1-17-66$

Program classification

Anelyst T.Curran; Eofillasana; H.C.Claass Date checked 1-17-66

Checked by V.J.Janzer

*Analyzed only when indicated by gross $\beta$ count.

Revised form 3-17-65
Checked from

Original Records

By.

Date $2-22-66$ 


\section{U. S. DEPT. OF THE INTERIOR .. GEOLOGICAL SURVEY \\ Statement of Water Analysis}

Source We11 J22-3

Location Lumberton, Lamar Co., Mississippi

SE $1 / 4$ NW $1 / 4 \ldots 1 / 4$ Sec _22, T T 2.N., R . 16 W. Field/Office No.

\begin{tabular}{l|l}
\hline Date Col. $\frac{10-26-65}{\text { USGS }}$ Time-1250_ Well Type Drilled Use Dom; stock & Ded \\
Col & Depth
\end{tabular}

Col. By USGS

Field detns: Tomp. $\left({ }^{\circ} \mathrm{F}\right)$

Sp. Cond. ( $\mu$ mhos )

Appearance $\mathrm{pH}$ Eh

Clear
Diam. (in.) $2 \quad$ Date drilled 1957

Water level (ft.) $40($ ? ) below 1.s.d., 1961

Discharge $8.18 \mathrm{gpm}, 5-14-63$

W. B. F. Quaternary (alluvium, terrace deposits, etc.)

Owner G. C. Saul,

Rt. 4, Lumberton, Miss.

\section{Physical characteristics} and computed values

\begin{tabular}{|c|c|c|}
\hline \multicolumn{3}{|c|}{ Chemical components } \\
\hline Silica $\left(\mathrm{SiO}_{2}\right)$ & 8.4 & \\
\hline Aluminum (AI) & $<.01$ & \\
\hline Iron $\left(F_{0}\right)$ & $<.01$ & \\
\hline Mangane se (Mn) & .02 & \\
\hline Calcium (Ca) & .3 & 0.01 \\
\hline Magne sium $(\mathrm{Mg})$ & .4 & .03 \\
\hline Strontium $\left(S_{r}\right)$ & .03 & -- \\
\hline Sodium (Na) & 2.7 & .12 \\
\hline Potassium (K) & .3 & .01 \\
\hline Lithium (Li) & $<.01$ & -- \\
\hline Copper ( $\mathrm{Cu})$ & .04 & -- \\
\hline $\operatorname{Zinc}(\mathrm{Zn})$ & .08 & -- \\
\hline
\end{tabular}

Dissolved Solids (ppm)

Res. on evap. of $180^{\circ} \mathrm{C}$

Calculated

Suspended solids (ppm)

Hardness os $\mathrm{CaCO}_{3}$ (ppm)

Total

Non-carbonate

Specific conductance

( $\mu$ mhos at $25^{\circ} \mathrm{C}$ )

$\mathrm{pH}$

Color

Percent sodium

SAR

\begin{tabular}{c}
21 \\
\hline 18 \\
\hline 2 \\
\hline 0 \\
17 \\
\hline 5.8 \\
\hline 71 \\
\hline .9 \\
\hline
\end{tabular}

Cations (epm)

0.17

Bicarbonate $\left(\mathrm{HCO}_{3}\right)$

Carbonate $\left(\mathrm{CO}_{3}\right)$

Sulfate $\left(\mathrm{SO}_{4}\right)$

Chloride (Cl)

Fluoride (F)

Nitrate $\left(\mathrm{NO}_{3}\right)$

Phosphate $\left(\mathrm{PO}_{4}\right)$

\section{Remarks:}

\begin{tabular}{lc}
3 & 0.05 \\
\hline 0 & .00 \\
\hline 1.0 & .02 \\
\hline 2.4 & .07 \\
\hline$<.1$ & -- \\
\hline 1.6 & .03 \\
\hline$<.01$ & -- \\
\hline
\end{tabular}

Anions (epm)

0.17

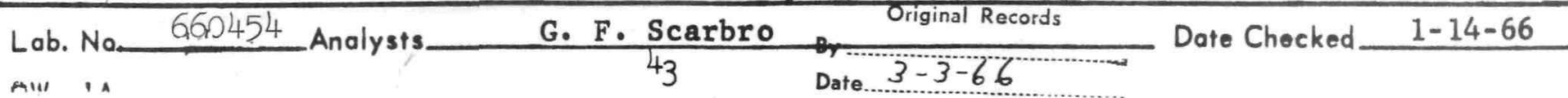


U. S. DEPT. OF THE INTERIOR--GEOLOGICAL SURVEY

Statement of Water Analysis - Radiochemical

Source

Well J22-3

Location Lumberton, Lamar Co., Mississippi

$\mathrm{SE} 1 / \mathrm{HW}^{1 / 4} 1 / 4 \mathrm{Sec} 22, \mathrm{~T}^{\mathrm{T}} 2 \mathrm{~N}, \mathrm{~N}^{\mathrm{R}} 16 \mathrm{~W}$. Field/Office No.

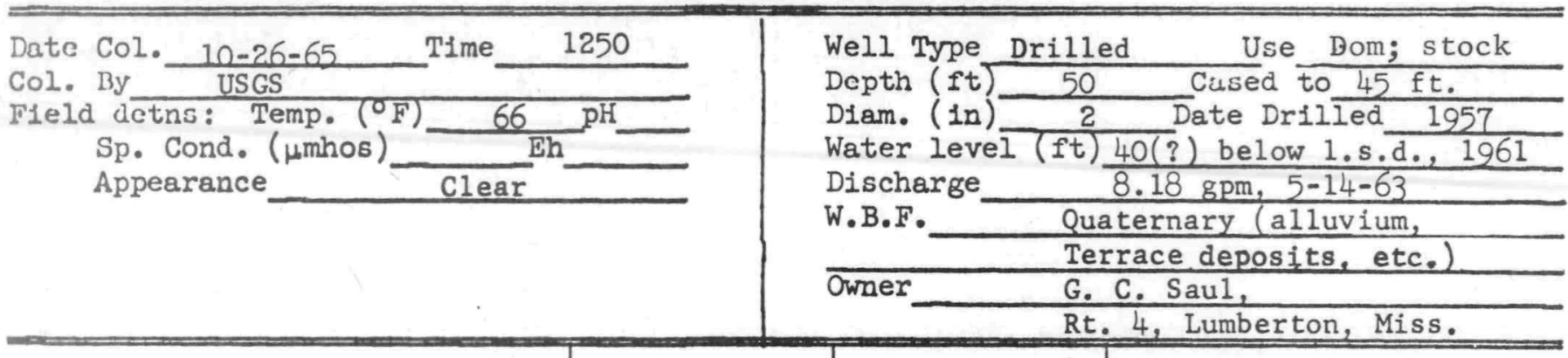

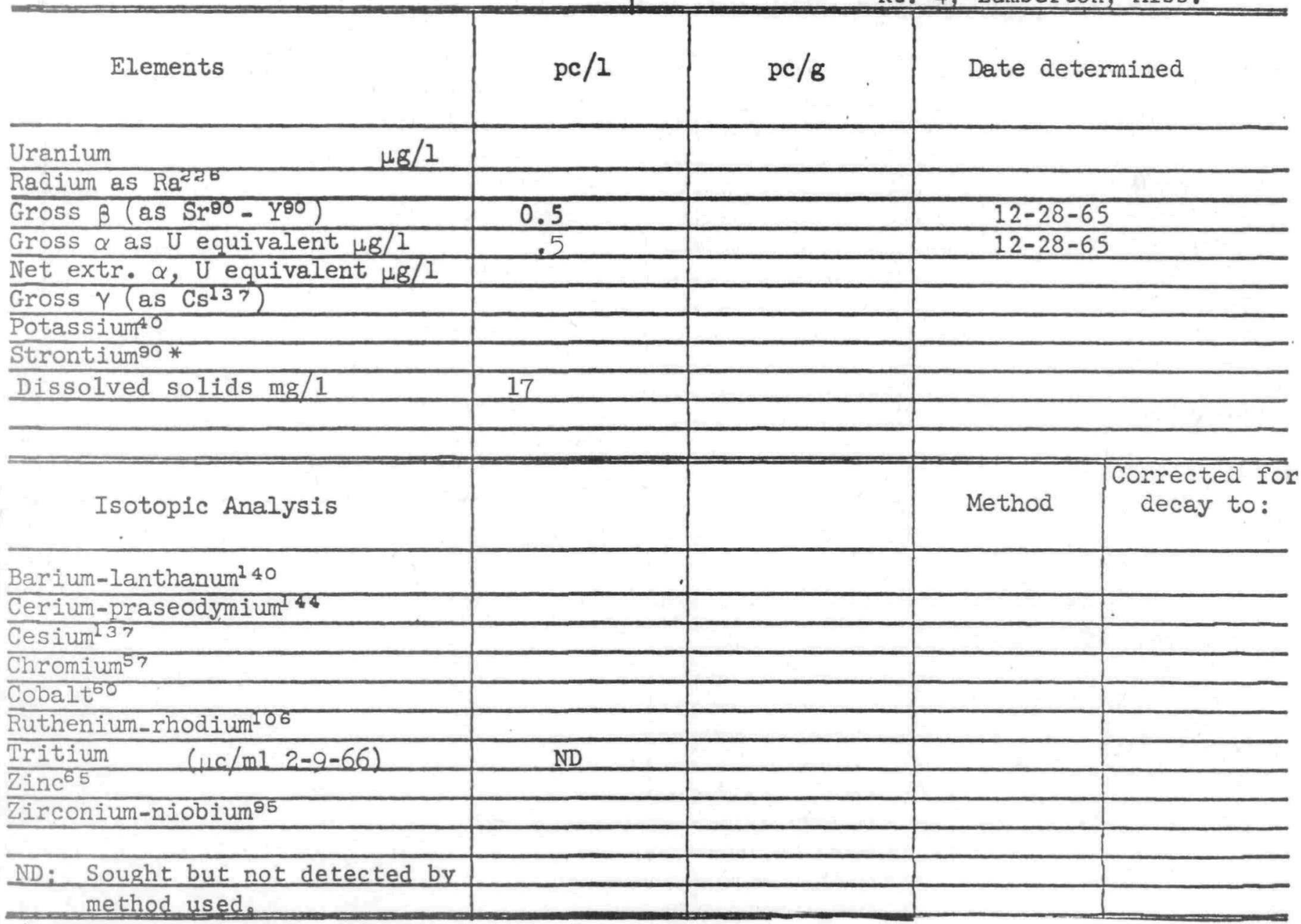

Date sample received $12-14-65$

Date data released $\frac{1-5-66}{1-66}$

Program classification

*Analyzed only when indicated by gross $\beta$ count.

Revised form 3-17-65
44
Analyst T.Curran; E.Villasana; H.C.Claasse Date checked 1-5-66

Checked by V.J. Janzer

Checked from

Original Records

By.

Date $2-22-66$

GPO 840.780 


\section{U. S. DEPT. OF THE INTERIOR .- GEOLOGICAL SURVEY \\ Statement of Water Analysis}

Source We11 J25-4

Location Purvis, Lamar Co., Mississippi

SE $1 / 4$ SE 1/4 NE 1/4 Sec.25, T. 2 N.er R. 16 W. Field/Office No.

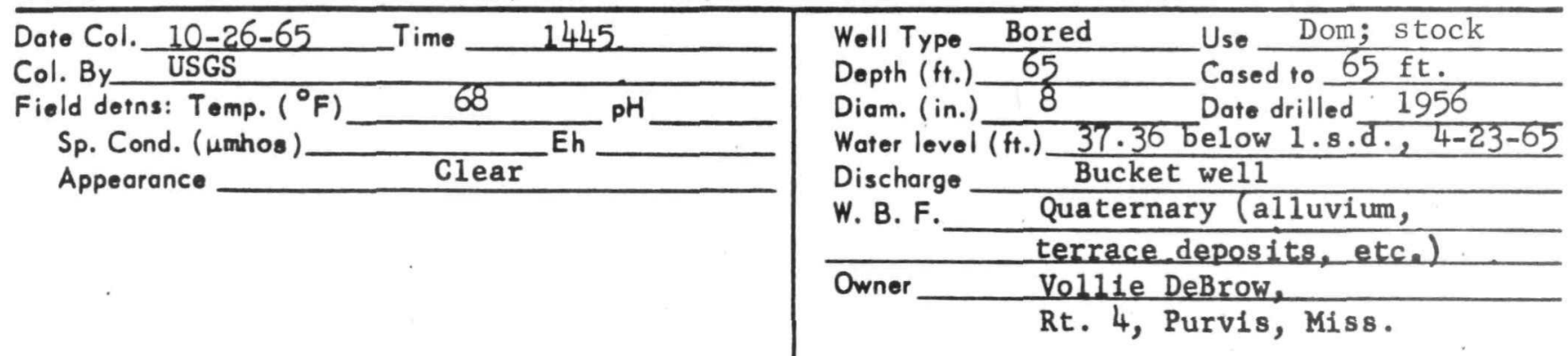

\begin{tabular}{|c|c|c|c|c|}
\hline \multicolumn{3}{|c|}{ Chemical components } & \multicolumn{2}{|c|}{$\begin{array}{l}\text { Physical characteristics } \\
\text { and computed values }\end{array}$} \\
\hline Silica $\left(\mathrm{SiO}_{2}\right)$ & 19 & & \multirow{3}{*}{$\begin{array}{l}\text { Dissolved Solids (ppm) } \\
\text { Res. on evap. at } 180^{\circ} \mathrm{C} \\
\text { Calculated }\end{array}$} & \\
\hline Aluminum (Al) & $\leq .01$ & & & 44 \\
\hline Iron $\left(F_{e}\right)$ & $<.01$ & & & 41 \\
\hline Manganese $(\mathrm{Mn})$ & $<.02$ & & \multirow{3}{*}{$\begin{array}{l}\text { Suspended solids (ppm) } \\
\text { Hardness as } \mathrm{CaCO}_{3}(\mathrm{ppm}) \\
\text { Total }\end{array}$} & \\
\hline Calcium $(\mathrm{Ca})$ & 3.9 & 0.19 & & \\
\hline Magnesium $(\mathrm{Mg})$ & .7 & .06 & & 13 \\
\hline Strontium (Sr) & .02 & $-\infty$ & \multirow{2}{*}{$\begin{array}{l}\text { Non-carbonate } \\
\text { Specific conductance }\end{array}$} & 0 \\
\hline Sodium (Na) & 3.2 & .14 & & \\
\hline Potassium (K) & .5 & .01 & \multirow{5}{*}{$\begin{array}{l}\left.\text { (umhos at } 25^{\circ} \mathrm{C}\right) \\
\text { Color } \\
\text { Percent sodium } \\
\text { SAR }\end{array}$} & 45 \\
\hline \multirow{6}{*}{$\begin{array}{l}\text { Lithium (Li) } \\
\text { Copper ( } \mathrm{Cu}) \\
\text { Zinc }(\mathrm{Zn})\end{array}$} & .01 & -- & & 6.1 \\
\hline & .04 & -- & & \\
\hline & .19 & -- & & 35 \\
\hline & & & & .4 \\
\hline & & & - & \\
\hline & Cations (epm) & 0.40 & \multicolumn{2}{|l|}{ Remarks: } \\
\hline
\end{tabular}

Bicarbonate $\left(\mathrm{HCO}_{3}\right)$

Carbonate $\left(\mathrm{CO}_{3}\right)$

Sulfate $\left(\mathrm{SO}_{4}\right)$

Chloride $(\mathrm{Cl})$

Fluoride (F)

Nitrate $\left(\mathrm{NO}_{3}\right)$

Phosphate $\left(\mathrm{PO}_{4}\right)$

\begin{tabular}{rr}
19 & 0.31 \\
\hline 0 & .00 \\
1.0 & .02 \\
\hline 2.9 & .08 \\
\hline .1 & -- \\
\hline .5 & .01 \\
\hline .01 & -- \\
\hline
\end{tabular}
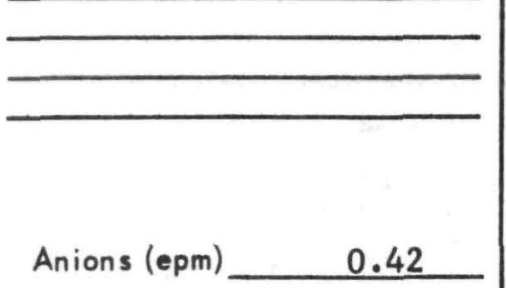

Well Type Bored

Depth ( ft.)

Water level (ft.) 37.36 below 1.s.d., 4-23-65

Discharge

Quaternary (alluvium,

terrace deposits, etc.)

Vol11e DeBrow.

Physical characteristics

and computed values 
U. S. DEPT. OF THE INTERIOR--GEOLOGICAL SURVEY

Statement of Water Analysis - Radiochemical

Source

We11 J25-4

Location Purvis, Lamar Co., Mississippi

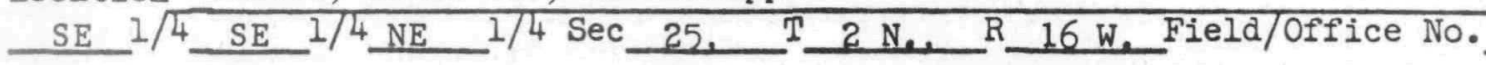

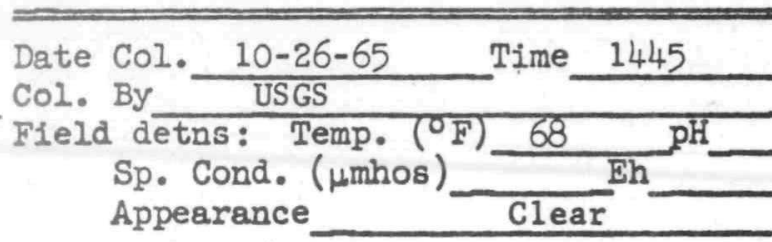

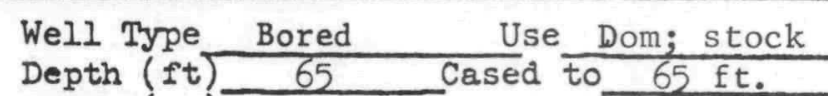

Diam. (In) Date Drilled 1956

Water level (ft) 37.36 below 1.s.d. 4-23-65

Discharge

W.B.F. Quaternary (alluvium,

owner terrace deposits, etc.)

\begin{tabular}{l} 
Owner $\frac{\text { Vollie DeBrow, }}{\text { Rt. 4, Purvis, Miss. }}$ \\
\hline
\end{tabular}

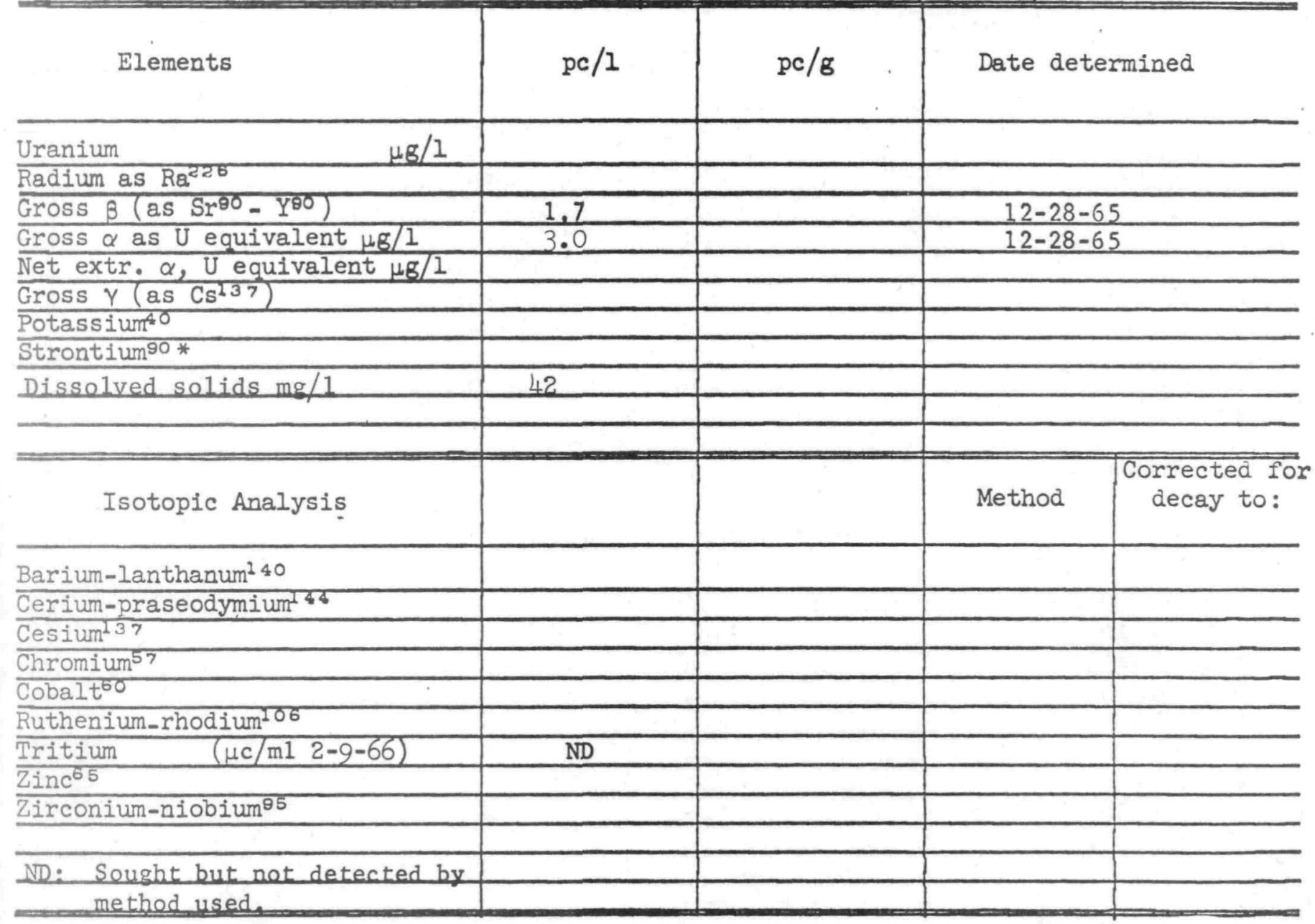

Date sample received $12-14-65$

Date data released $1-5-66$

Program classification

*Analyzed only when indicated by gross $\beta$ count.

Revised form 3-17-65
46
Analyst T.Curran; E.Villasana; H.C.Claass Date checked 1-5-66

Checked by V.J. Janzer

\section{Checked from}

Original Records

By...

Date. $2-2-66$ 


\section{U. S. DEPT. OF THE INTERIOR .- GEOLOGICAL SURVEY \\ Statement of Water Analysis}

Source We11 J25-5

Location Purvis, Lamar Co., Mississipp1

SE $1 / 4$ NE $1 / 4$ SE $1 / 4$ Sec $-25, \quad T \cdot 2 \mathrm{~N} ., \mathrm{R}, 16 \mathrm{~W}$. Field/Office No.

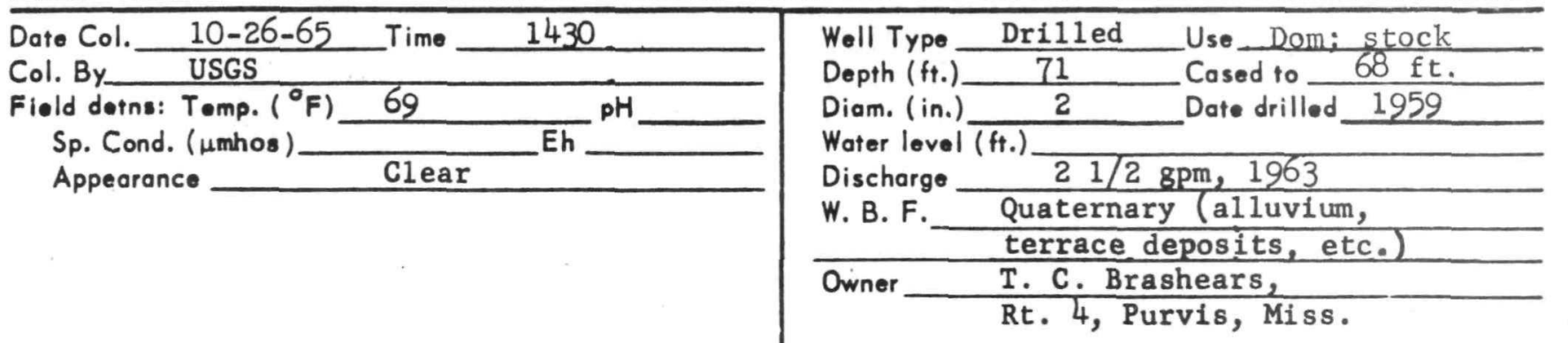

\begin{tabular}{|c|c|c|c|c|}
\hline & $\begin{array}{c}\text { Chemical components } \\
\text { ppm }\end{array}$ & epm & \multicolumn{2}{|c|}{$\begin{array}{l}\text { Physical characteristics } \\
\text { and computed values }\end{array}$} \\
\hline \multirow{3}{*}{$\begin{array}{l}\text { Silica }\left(\mathrm{SiO}_{2}\right) \\
\text { Aluminum }(\mathrm{Al}) \\
\text { Iron }\left(\mathrm{F}_{\bullet}\right)\end{array}$} & 8.4 & & \multirow{5}{*}{$\begin{array}{l}\text { Dissolved Solids (ppm) } \\
\text { Res. on evap. at } 180^{\circ} \mathrm{C} \\
\text { Calculated } \\
\text { Suspended solids (ppm) } \\
\text { Hardness as } \mathrm{CaCO}_{3}(\mathrm{ppm}) \\
\text { Total }\end{array}$} & \\
\hline & $\frac{<.01}{<.01}$ & & & $\frac{20}{18}$ \\
\hline & $\frac{<.01}{.05}$ & & & \\
\hline Calcium (Ca) & .3 & 0.01 & & \\
\hline Magnesium (Mg) & .5 & .04 & & 3 \\
\hline Strontium ( $\mathrm{Sr}$ ) & $<.02$ & -- & \multirow{7}{*}{$\begin{array}{l}\text { Non-carbonate } \\
\text { Specific conductance } \\
\quad\left(\mu \text { mhos at } 25^{\circ} \mathrm{C} \text { ) }\right. \\
\text { pH } \\
\text { Color } \\
\text { Percent sodium } \\
\text { SAR }\end{array}$} & 0 \\
\hline Sodium ( $\mathrm{Na}$ ) & 2.6 & .11 & & \\
\hline Potassium (K) & .3 & .01 & & 19 \\
\hline Lithium (Li) & .01 & -- & & 5.7 \\
\hline Copper ( $\mathrm{Cu})$ & .05 & -- & & \\
\hline \multirow[t]{3}{*}{ Zinc $(\mathrm{Zn})$} & .13 & -- & & 65 \\
\hline & & & & .7 \\
\hline & Cations (epm) & 0.17 & Remarks: & \\
\hline
\end{tabular}

Bicarbonate $\left(\mathrm{HCO}_{3}\right)$

Carbonate $\left(\mathrm{CO}_{3}\right)$

Sulfate $\left(\mathrm{SO}_{4}\right)$

Chloride (CI)

Fluoride (F)

Nitrate $\left(\mathrm{NO}_{3}\right)$

Phosphate $\left(\mathrm{PO}_{4}\right)$

\begin{tabular}{cc}
5 & 0.08 \\
\hline 0 & .00 \\
\hline$<. I$ & -- \\
\hline 2.9 & .08 \\
\hline$<.1$ & -- \\
\hline .9 & .01 \\
\hline$<.01$ & -- \\
\hline
\end{tabular}

Anions (epm) $\quad 0.17$ 
Lab. No. 660456

U. S. DEPT. OF THE INTERIOR--GEOLOGICAL SURVEY

Statement of Water Analysis - Radiochemical

Source

We11 J25-5

Location Purvis, Lamar Co., Mississippi

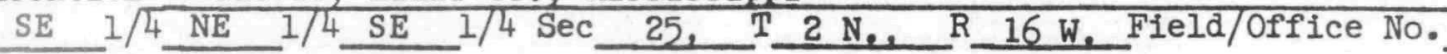

Dete

Date Col. 10-26-65 Time $1430 \quad$ Well Type Drilled Use Dom; stock

Col. By USGS Depth (ft 71 Cased to $68 \mathrm{ft}$.

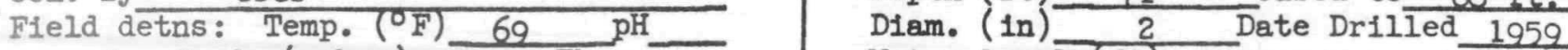

$\mathrm{Sp}$. Cond. ( $\mu$ mhos) Wh Water level (ft) Unknown

Appearance

Clear

Discharge $2 1 \longdiv { 2 \mathrm { gpm } , 1 9 6 3 }$

W.B.F. Quaternary (alluvium,

terrace deposits, etc.)

Owner T. C. Brashears,

Rt. 4, Purvis, Miss.

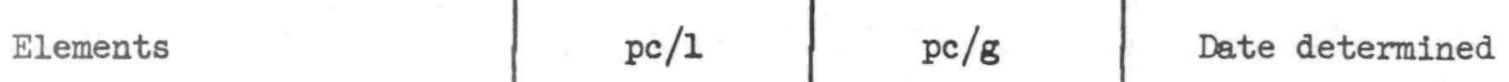

Uranium

Radium as Racro

Gross $\beta\left(\right.$ as $\left.\mathrm{Sr}^{90}-\mathrm{Y}^{80}\right)$

Gross $\alpha$ as U equivalent $\mu g / 1$

Net extr. $\alpha$, U equivalent $\mu \mathrm{g} / 1$

Gross $Y$ (as $\left.\mathrm{Cs}^{137}\right)$

Potassium ${ }^{40}$

Strontium90*

Dissolved solids $\mathrm{mg} / 1$

$\mu \mathrm{g} / 1$

\section{Dissolved solids mg/ 1}

Isotopic Analysis

Barium-lanthanum ${ }^{140}$

Cerium-praseodymium ${ }^{14}$

Cesium ${ }^{3}$ ?

Chromium ${ }^{5}$

Cobalt ${ }^{\mathrm{CO}}$

Ruthenium-rhodium ${ }^{106}$

Tritium ( $\mu c / m 12-9-66)$

Zinc ${ }^{65}$

Zirconium-niobium ${ }^{95}$

ND: Sought but not detected by method used.

Date sample received 12-14-65

Date data released 1-17-66

Program classification

*Analyzed only when indicated by gross $\beta$ count.

Revised form 3-17-65
48

Analyst T.Curran; E.Villasana; H.C.Claasse Date checked 1-17-66

Checked by V. J. Janzer

Corrected for

Method decay to:

$1-8-66$ $1-8-66$

\footnotetext{
Chicked from

Criginal Records

By

Date $2-22-66$
} 


\section{U. S. DEPT. OF THE INTERIOR .. GEOLOGICAL SURVEY \\ Statement of Water Analysis}

Source Wel1 J26-2

Location Lumberton, Lamar Co., Mississippi

NE $1 / 4$ NE $1 / 4 \ldots 1 / 4$ Sec. $26, T, 2 N ., R, 16$ W. Field/Office No.

\begin{tabular}{|c|c|}
\hline \multirow{6}{*}{ 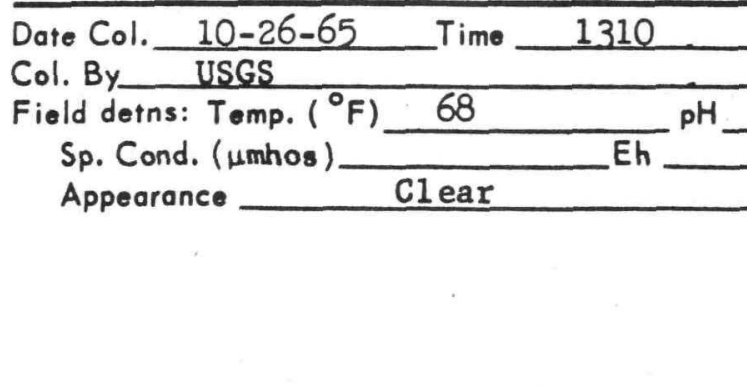 } & \multirow{5}{*}{ 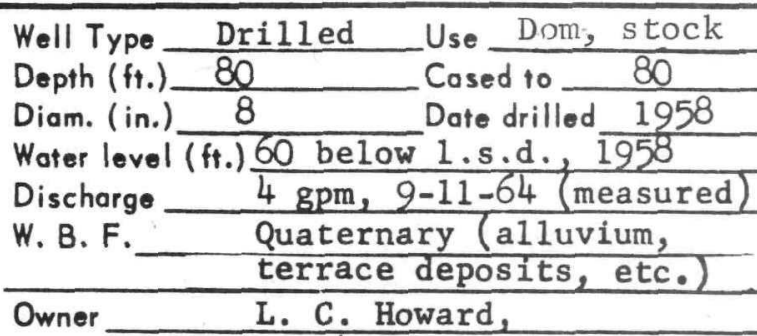 } \\
\hline & \\
\hline & \\
\hline & \\
\hline & \\
\hline & Owner $\frac{\text { L. C. Howard, }}{\text { Rt. 4, Lumberton, Miss. }}$ \\
\hline
\end{tabular}

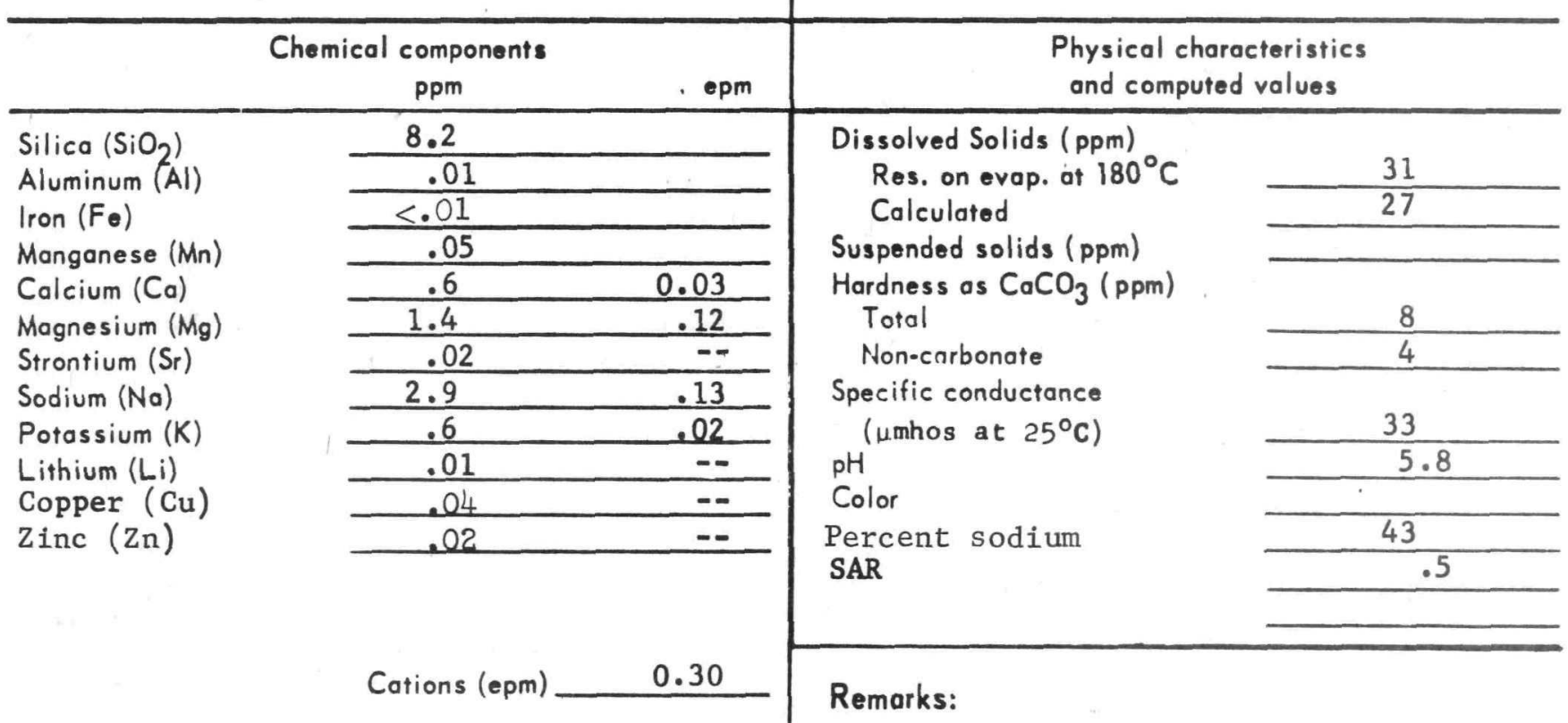

Bicarbonate $\left(\mathrm{HCO}_{3}\right)$

Carbonate $\left(\mathrm{CO}_{3}\right)$

Sulfate $\left(\mathrm{SO}_{4}\right)$

Chloride $(\mathrm{Cl})$

Fluoride (F)

Nitrate $\left(\mathrm{NO}_{3}\right)$

Phosphate $\left(\mathrm{PO}_{4}\right)$

\begin{tabular}{cc}
4 & 0.07 \\
\hline 0 & .00 \\
\hline 1.0 & .02 \\
\hline 4.0 & .11 \\
\hline$<.1$ & -- \\
\hline 5.8 & .09 \\
\hline$<.01$ & -- \\
\hline
\end{tabular}

Anions (epm) $\quad 0.29$

Lab. No.

Analysts

G. F. Scarbro 
Lab. No. 660457

U. S. DEPT. OF THE INTERIOR--GEOLOGICAL SURVEY

Statement of Water Analysis - Radiochemical

Source

We11 J26-2

Location Lumberton, Lamar Co., Mississippi

Date Col. 10-26-65 Time $1310 \quad$ Well Type Drilled Use Dom; stock

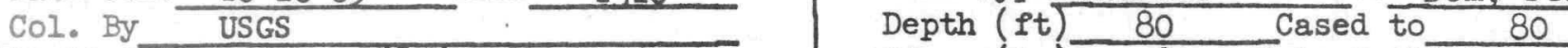

Field detns: Temp. ( $\left.{ }^{\circ} \mathrm{F}\right) 68$ pH $\quad$ Diam. (in) 8 Date Drilled 1958

Sp. Cond. ( $\mu$ mhos) Eh - Water level (ft) 60 below 1.s.d., 1958

Appearance

Clear

Discharge $4 \mathrm{gpm}, 9-11-64$ (measured)

W.B.F. Quaternary (alluvium, terrace deposits, etc.)

Owner_L. C. Howard,

Rt. 4, Lumberton, Miss.

\begin{tabular}{|c|c|c|c|c|}
\hline Elements & $\mathrm{pc} / 1$ & $\mathrm{pc} / \mathrm{g}$ & \multicolumn{2}{|c|}{ Date determined } \\
\hline \multicolumn{5}{|l|}{ Uranium $\mu \mathrm{g} / 1$} \\
\hline \multicolumn{5}{|l|}{ Radium as $\mathrm{Ra}^{\mathrm{L}} \mathrm{L}$} \\
\hline Gross $\beta\left(\right.$ as Sr90 $\left.-Y^{90}\right)$ & 1.7 & & \multicolumn{2}{|c|}{$1-8-66$} \\
\hline Gross $\alpha$ as U equivalent $\mu g / 1$ & 1.5 & & \multicolumn{2}{|c|}{$1-8-66$} \\
\hline \multicolumn{5}{|l|}{ Net extr. $\alpha$, U equivalent $\mu g / 1$} \\
\hline \multicolumn{5}{|l|}{ Gross $Y\left(\right.$ as $\left.\operatorname{Cs}^{137}\right)$} \\
\hline \multicolumn{5}{|l|}{ Potassium 40} \\
\hline \multicolumn{5}{|l|}{ Strontium90* } \\
\hline \multicolumn{5}{|l|}{ Dissolved solids $\mathrm{mg} / 1$} \\
\hline \multicolumn{5}{|l|}{ 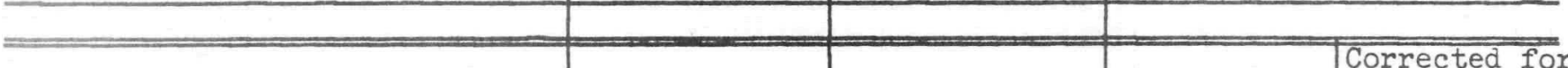 } \\
\hline Isotopic Analysis & & & Method & $\begin{array}{l}\text { Corrected for } \\
\text { decay to: }\end{array}$ \\
\hline \multicolumn{5}{|l|}{ Barium-lanthanum ${ }^{1} 40$} \\
\hline \multicolumn{5}{|l|}{ Cerium-praseodymium ${ }^{14}$} \\
\hline \multicolumn{5}{|l|}{ Cesiumª } \\
\hline \multicolumn{5}{|l|}{ Chromium ${ }^{57}$} \\
\hline \multicolumn{5}{|l|}{ Cobalt 60} \\
\hline \multicolumn{5}{|l|}{ Ruthenium_rhodium ${ }^{106}$} \\
\hline Tritium $(u \mathrm{c} / \mathrm{m} 12-9-66)$ & ND & & & \\
\hline \multicolumn{5}{|l|}{ Zinc $^{65}$} \\
\hline \multicolumn{5}{|l|}{ Zirconium-niobium ${ }^{95}$} \\
\hline & & & & \\
\hline \multicolumn{5}{|l|}{ ND: Sought but not detected by } \\
\hline method used. & & & & \\
\hline
\end{tabular}

Date sample received 12-14-65

Date data released $1-17-66$

Program classification

*Analyzed only when indicated by gross $\beta$ count.

Revised form 3-17-65
50

Analyst T.Curran; E.Villasana; H.C.Claass Date checked

Checked by $1-17-66$

V. J. Janzer

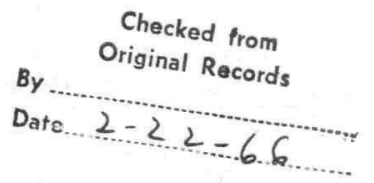

Checked from

By.

$2-22-66$

GPO $848 \cdot 788$ 


\section{U. S. DEPT. OF THE INTERIOR -. GEOLOGICAL SURVEY \\ Statement of Water Analysis}

Source We11 J27-1

Location Lumberton, Lamar Co., Mississippi

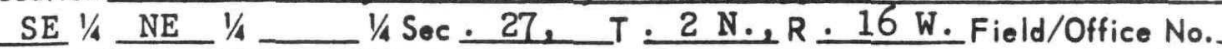

Date Col. 10-26-65 Time 1320 Well Type Drilled Use Dom; stock

Col. By USGS

Field detns: Temp. $\left({ }^{\circ} \mathrm{F}\right)$

Sp. Cond. (umhos)

Appearance $\mathrm{pH}$ Clear
Depth (ft.) $\quad 39$

Diam. (in.) 8 Cased to $38.41 \mathrm{ft}$.

Water level (ft.) 38.62 below 1.s. d. 4-23-65 Discharge Bucket well

W. B. F. Quaternary (alluvium, terrace deposits, etc.)

Owner Hubert Powell. Rt. 4, Lumberton, Miss.

\begin{tabular}{|c|c|c|}
\hline \multicolumn{3}{|c|}{ Chemical components } \\
\hline Silica $\left(\mathrm{SiO}_{2}\right)$ & 8.6 & \\
\hline Aluminum (AI) & .01 & \\
\hline Iron $\left(F_{e}\right)$ & $<.01$ & \\
\hline Manganese $(M n)$ & .01 & \\
\hline Calcium (Ca) & 2.1 & 0.10 \\
\hline Magnesium (Mg) & .3 & .02 \\
\hline Strontium (Sr) & .02 & $=-$ \\
\hline Sodium (Na) & 3.0 & .13 \\
\hline Potassium (K) & .4 & .01 \\
\hline Lithium (Li) & .01 & -- \\
\hline Copper (Cu) & $<.01$ & -- \\
\hline Zinc $(\mathrm{Zn})$ & .08 & -- \\
\hline
\end{tabular}

Physical characteristics and computed values

Dissolved Solids (ppm)

Res. on evap. at $180^{\circ} \mathrm{C}$

Caleulated

Suspended solids (ppm)

Hardness os $\mathrm{CaCO}_{3}$ (ppm)

Total

Non-carbonate

Specific conductance

( $\mu$ mhos at $25^{\circ} \mathrm{C}$ )

$\mathrm{pH}$

Color

Percent. sodium

SAR

$\begin{array}{r}27 \\ \hline 25 \\ \hline \\ 6 \\ \hline 0\end{array}$

$\begin{array}{r}26 \\ \hline 6.1 \\ \hline 50 \\ \hline .5\end{array}$

Cations (epm) $\quad 0.26 \quad$ Remarks:

Bicarbonate $\left(\mathrm{HCO}_{3}\right)$

Corbonate $\left(\mathrm{CO}_{3}\right)$

Sulfate $\left(\mathrm{SO}_{4}\right)$

Chloride (Cl)

Fluoride (F)

Nitrate $\left(\mathrm{NO}_{3}\right)$

Phosphate $\left(\mathrm{PO}_{4}\right)$

\begin{tabular}{cc}
10 & 0.16 \\
\hline 0. & .00 \\
\hline 1.0 & .02 \\
\hline 2.2 & .06 \\
\hline$<.1$ & -- \\
\hline 2.1 & .03 \\
\hline$<.01$ & -- \\
\hline
\end{tabular}

Civeked from

Anions (epm)

0.27 
U. S. DEPT. OF THE INTERIOR--GEOLOGICAL SURVEY

Statement of Water Analysis - Radiochemical

Source

We11 J27-1

Location Lumberton, Lamar Co., Mississippi
$\mathrm{SE} 1 / 4 \mathrm{NE} 1 / 4$
$1 / 4 \mathrm{Sec} .27$, T.2 N., R. 16 W. Field/Office No.

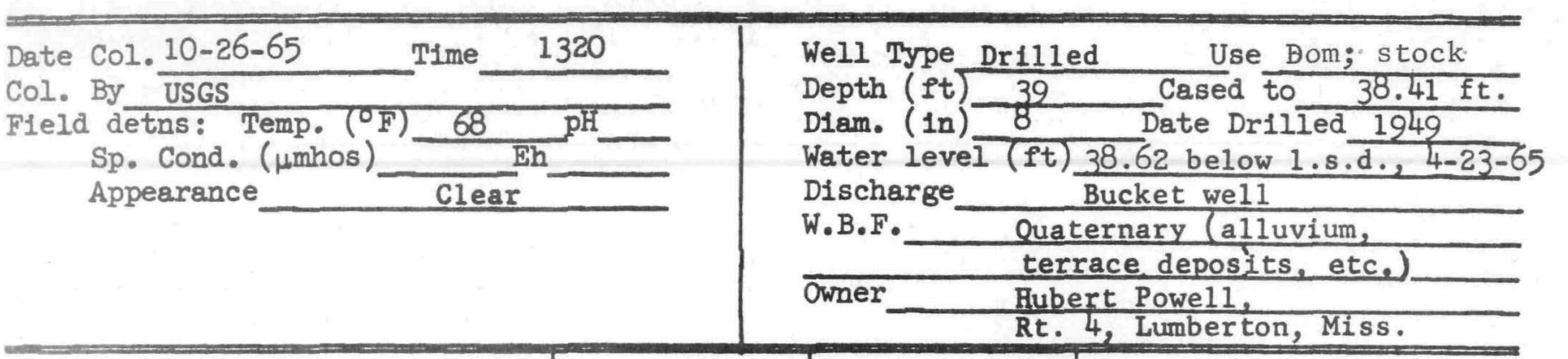

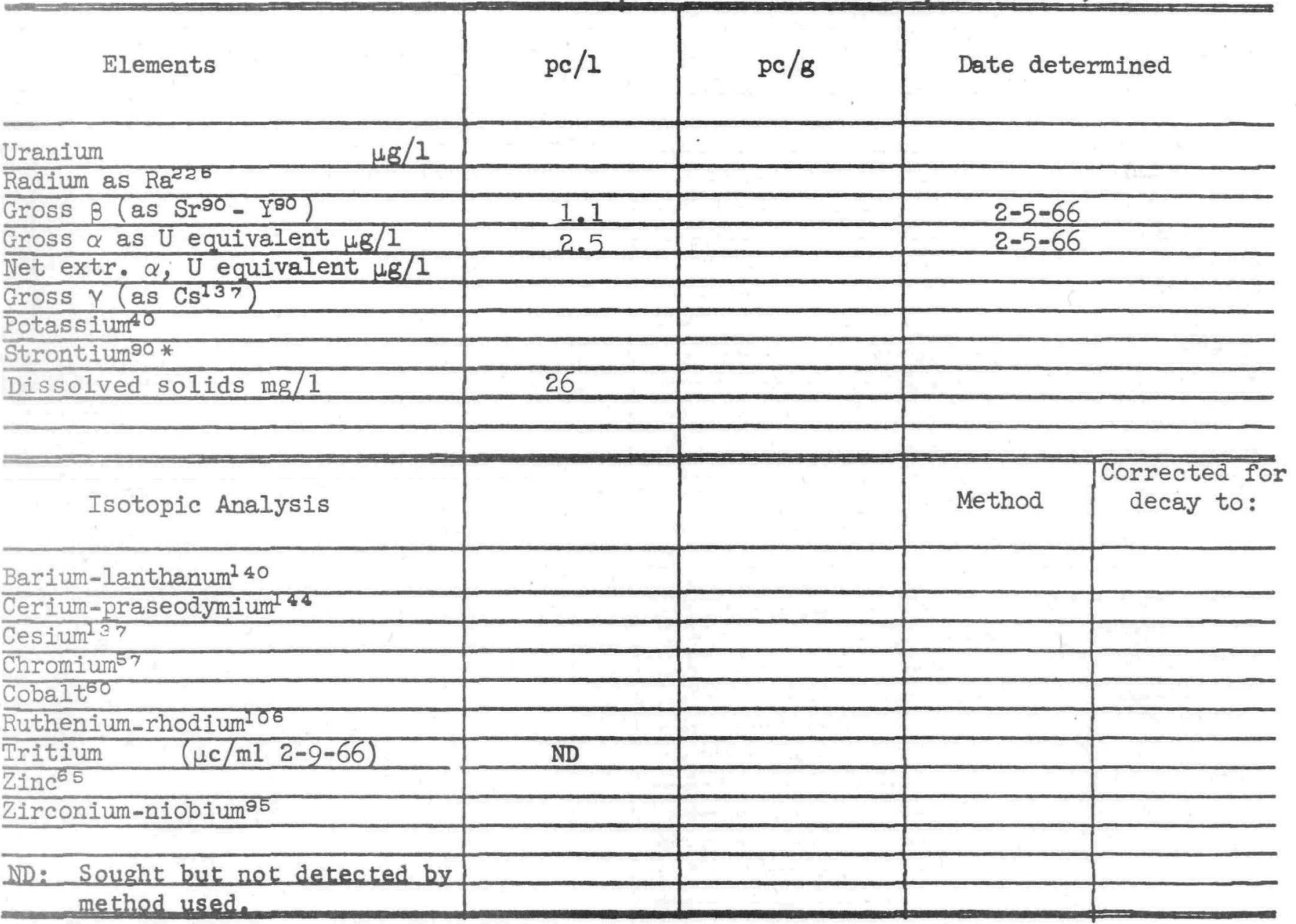

Date sample received 12-14-65

Date data released $2-11-66$

Program classification
Anelyst P.K.Roscio; T.Curran; H.C.Claassen Date checked 2-11-66

Checked by V. J. Janzer

*Analyzed only when indicated by gross 8 count.

Revised form 3-17-65 


\section{U. S. DEPT. OF THE INTERIOR -. GEOLOGICAL SURVEY \\ Statement of Water Analysis}

Source We11 J32-1

Location Lumberton, Lamar Co., Mississippi

NE $1 / 4$ NW $1 / 4 \ldots 1 / 4$ Sec. 32 , T, 2 N., R. 16 W. Field/Office No.

Date Col. 10-26-65 Time 1355

Col. By USGS

Field detns: Tomp. $\left({ }^{\circ} \mathrm{F}\right)$

Sp. Cond. (umhos)

Appearance

64

$\mathrm{pH}$

Eh

Clear
Well Type Drilled

Depth (ft.) 454

Diam. (in.) 2

Water level ( $\mathrm{ft}$.) Unknown

Discharge A few gpm (estimated)

W. B. F. Miocene (sands and small grave1)

Owner John Q. Butler, Rt. 4, Lumberton, Miss.

\begin{tabular}{|c|c|c|}
\hline \multicolumn{3}{|c|}{ Chemical components } \\
\hline & ppm & epm \\
\hline Silica $\left(\mathrm{SiO}_{2}\right)$ & 43 & \\
\hline Aluminum ( $\mathrm{Al})$ & $\leq .01$ & \\
\hline Iron $\left(F_{e}\right)$ & $<, 01$ & \\
\hline Manganese $(M n)$ & .17 & \\
\hline Calcium (Ca) & 16 & 0.80 \\
\hline Magnesium (Mg) & 3.3 & .27 \\
\hline Strontium (Sr) & .33 & -- \\
\hline Sodium $(\mathrm{Na})$ & 27 & 1.17 \\
\hline Potassium (K) & 2.6 & .07 \\
\hline Lithium (Li) & .04 & -- \\
\hline Copper $(\mathrm{Cu})$ & .03 & -- \\
\hline $\operatorname{Zinc}(\mathrm{Zn})$ & .39 & -- \\
\hline
\end{tabular}

\section{Physical characteristics}

and computed values

Silica $\left(\mathrm{SiO}_{2}\right)$

Aluminum (Al)

Iron (Fe)

Manganese $(M n)$

Calcium ( $\mathrm{Ca})$

Magnesium (Mg)

Strontium ( $\mathrm{Sr}$ )

Sodium $(\mathrm{Na})$

Potassium (K)

Lithium ( $\mathrm{Li}$ )

Zinc ( $\mathrm{Zn}$ )
Cations (epm)

2.33
Dissolved Solids (ppm)

Res. on evap. at $180^{\circ} \mathrm{C}$

Calculated

Suspended solids (ppm)

Hardness as $\mathrm{CaCO}_{3}$ (ppm)

Total

Non-carbonate

Specific conductance

( $\mu$.mhos at $25^{\circ} \mathrm{C}$ )

$\mathrm{pH}$

Color

Percent sodium

SAR

$\begin{array}{r}162 \\ \hline 164 \\ \hline \\ 54 \\ \hline 0\end{array}$

7.0

50

\section{Bicarbonate $\left(\mathrm{HCO}_{3}\right)$}

Carbonate $\left(\mathrm{CO}_{3}\right)$

Sulfote $\left(\mathrm{SO}_{4}\right)$

Chloride (CI)

Fluoride (F)

Nitrate $\left(\mathrm{NO}_{3}\right)$

Phosphate $\left(\mathrm{PO}_{4}\right)$

\begin{tabular}{cc}
126 & 2.07 \\
\hline 0 & .00 \\
\hline 2.0 & .04 \\
\hline 7.6 & .21 \\
\hline$<.1$ & -0 \\
\hline .1 & .00 \\
\hline$<.01$ & - \\
\hline & \\
\hline
\end{tabular}

Anions (epm)

2.32

\section{Remarks:}


Lab. No. 660459

U. S. DEPT. OF THE INTERIOR--GEOLOGICAL SURVEY

Statement of Water Analysis - Radiochemical

Source

We11 J32-1

Location Lumberton, Lamar Co, Mississippi $\mathrm{NE} 1 / 4 \mathrm{NW} 1 / 4 \quad 1 / 4 \mathrm{Sec} 32 \mathrm{~T}$ ? N. Ne. R 16 W. Field/Office No. Use Date Col. 10-26-65 Time 1355 Col. By USGS Fleld detns: Temp. (oF) Sp. Cond. ( $\mu$ mhos ) Appearance $64 \mathrm{pH}$ Eh Clear

Well Type Drilled Use Dom; stock

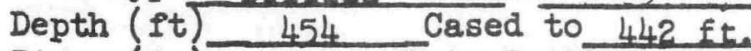
Diam. (In) 2 Date. Drilled June 1956 Water level (ft) Unknown

Discharge A few gpm (estimated) W.B.F. Miocene (sands and small gravel)

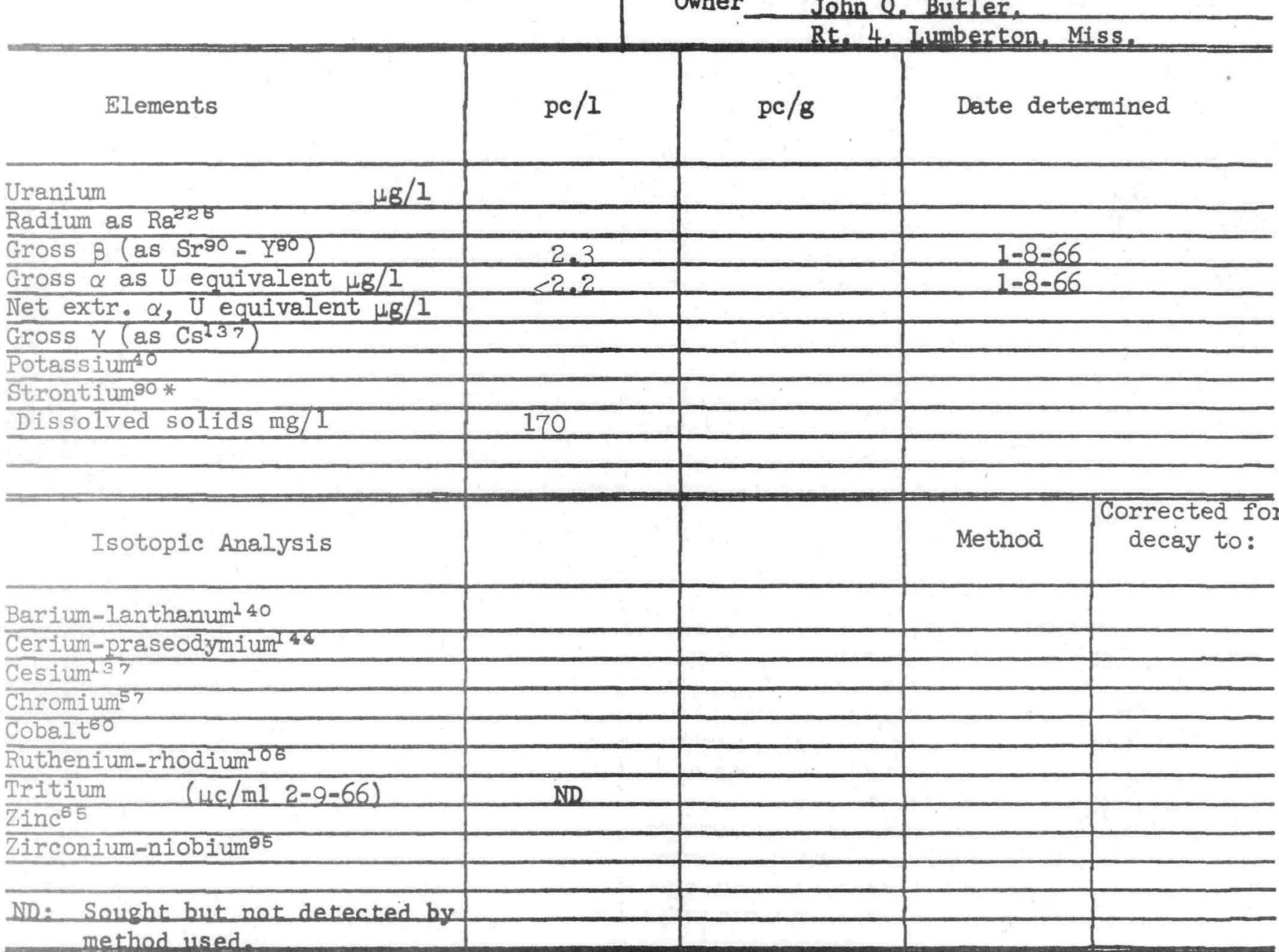

Date sample received 12-14-65

Date data released $1-17-66$

Program classification

*Analyzed only when indicated by gross $\beta$ count.

Revised form 3-17-65
Analyst T.Curran: P.K.Roscio: H.C.Claasse Date checked 1-17-66 Checked by V. J. Janzer 


\section{U. S. DEPT. OF THE INTERIOR .. GEOLOGICAL SURVEY Statement of Water Analysis}

Source

We11 J34-2

Location Baxterville, Lamar Co., Mississippi

NW $1 / 4$ SE $1 / 4 \ldots 1 / 4$ Sec $-34,-T-2 N, R, 16$ W. Field/Office No.

Date Col. 10-26-65 Time 1340 Well Type Drilled

Col. By USGS

Field derns: Temp. $\left({ }^{\circ} \mathrm{F}\right) \quad 63 \quad \mathrm{pH}$

Sp. Cond. (umhos)

Appearance

Clear

Well Type 132
Depth $(f t) \quad$.13

Diom. (in.)

4

Water level (ft.) 68.72 below 1.s.d, 8-14-61

Discharge

W. B. F $25 \mathrm{gpm}$

Quaternary (alluvium, terrace deposits, etc.)

Owner Kerry Kittre11, Baxterville, Miss.

\section{Physical characteristics} and computed values

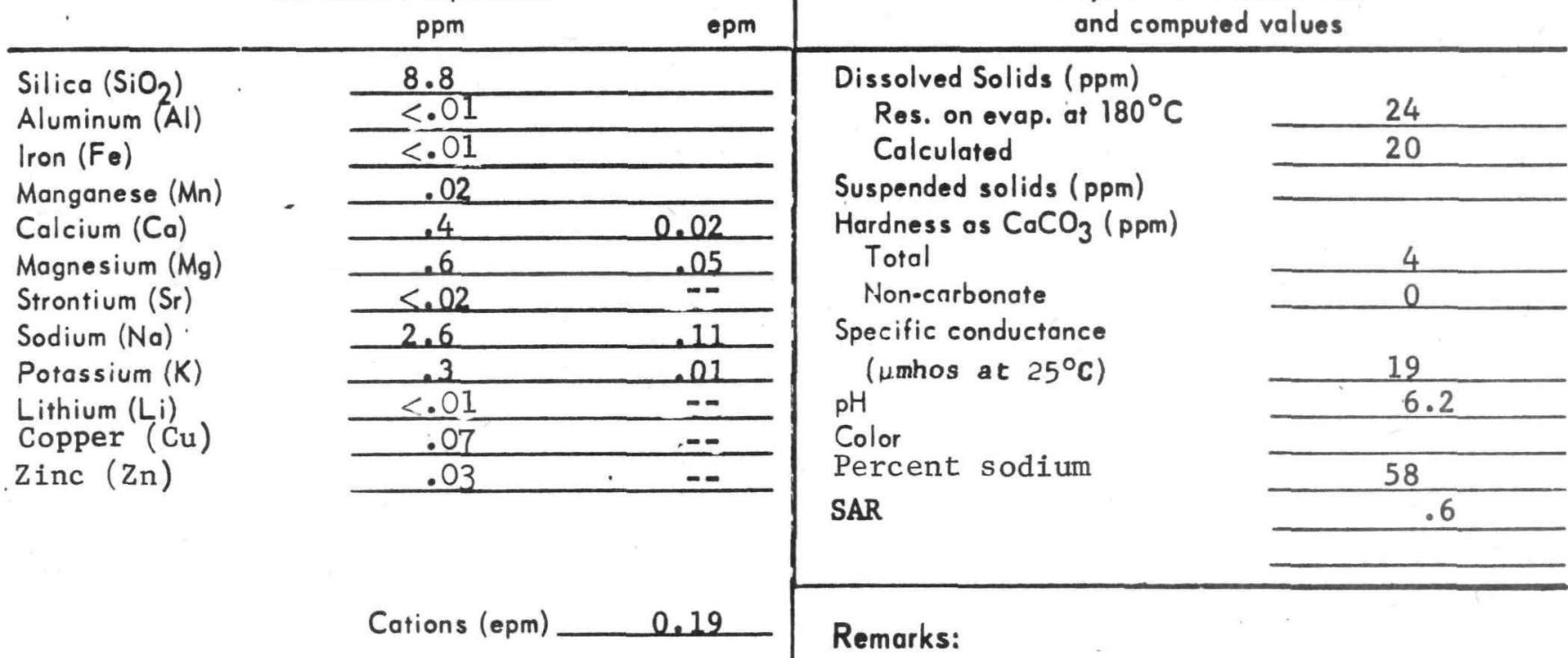

Bicarbonate $\left(\mathrm{HCO}_{3}\right)$

Carbonate $\left(\mathrm{CO}_{3}\right)$

Sulfate $\left(\mathrm{SO}_{4}\right)$

Chloride (Cl)

Fluoride ( $F$ )

Nitrate $\left(\mathrm{NO}_{3}\right)$

Phosphate $\left(\mathrm{PO}_{4}\right)$

\begin{tabular}{lc}
5 & 0.08 \\
\hline 0 & .00 \\
\hline 1.0 & .02 \\
\hline 2.4 & .07 \\
\hline$<.1$ & -- \\
\hline 1.6 & .03 \\
\hline$<.01$ & -- \\
\hline & \\
\hline & \\
\hline
\end{tabular}

Anions (epm) $0.20^{\circ}$ 
U. S. DEPT. OF THE INTERIOR--GEOLOGICAL SURVEY

Statement of Water Analysis - Radiochemical

Source

We11 J34-2

Location Baxterville, Lamar Co., Mississippi
NW $1 / 4$ SE $1 / 4$
$1 / 4 \mathrm{Sec} 34$, T2 N.,

(

Date Col. 10-26-65 Time 1340

Col. By USGS

Field detns: Temp. ( $\left.{ }^{\circ} F\right)$

Sp. Cond. ( $\mu$ mhos)

Appearance

63

Clear

Eh

مH

$\longrightarrow$

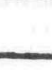
- $-$

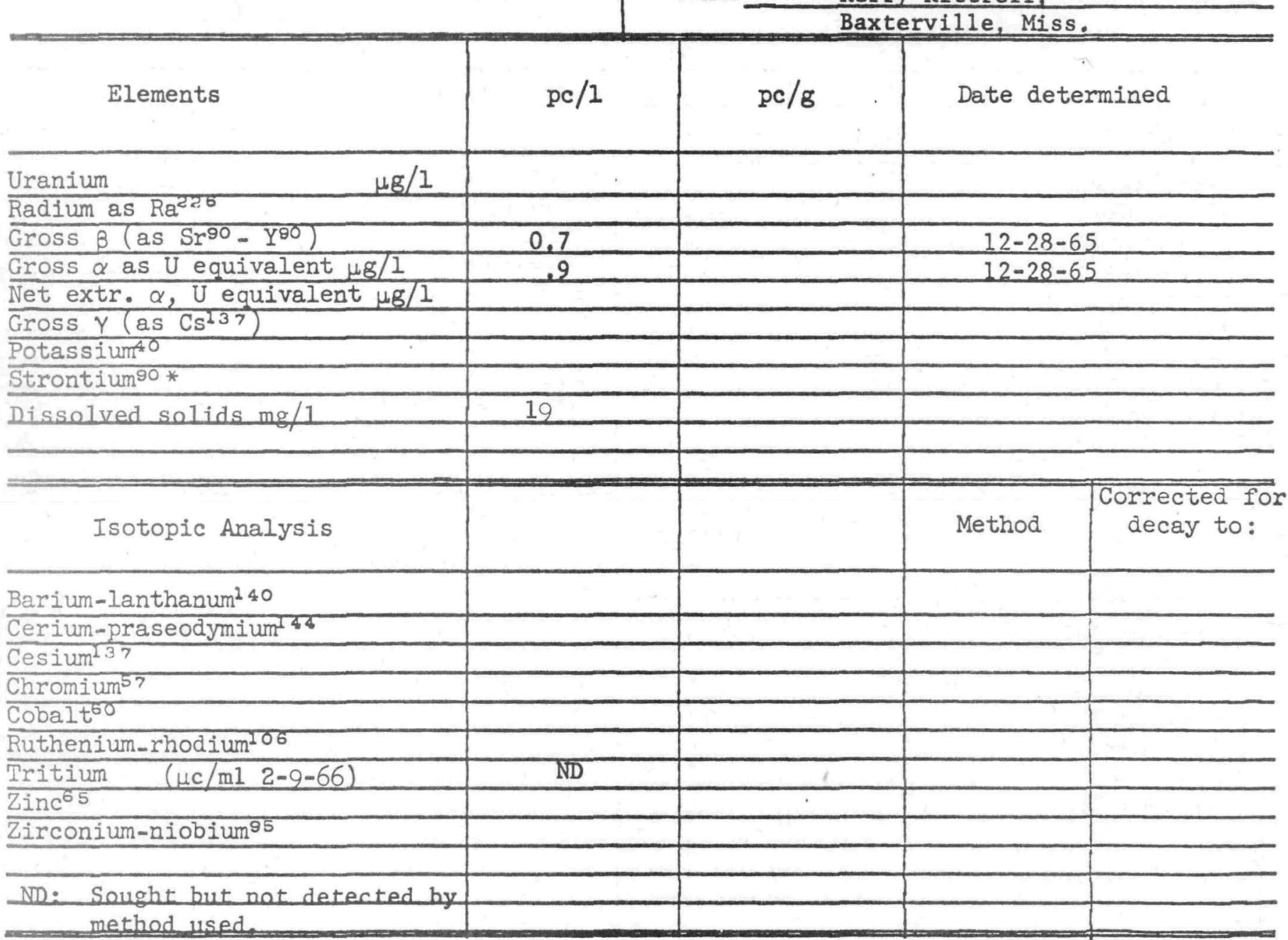

Date sample received 12-14-65

Date data released $1-5-66$

Program classification

*Analyzed only when indicated by gross $\beta$ count. Revised form $3-17-65$
Well Type Drilled Use Dom; stock; Ind

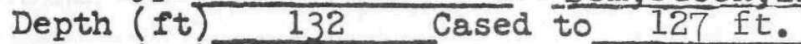

Diam. (in) 4 Date Drilled Oct. 1960

Water level (ft) 68.72 below 1.s.d., 8-14-61

Discharge $25 \mathrm{gpm}$

W.B.F. Quaternary (alluvium, terrace deposits, etc.)

Owner_Kerry Kittrell,

Baxterville, Miss.

Analyst T.Curran; P.K.Roscio; H.C.Claasse Date checked 1-5-66

Checked by V.J. Janzer 


\section{U. S. DEPT. OF THE INTERIOR -- GEOLOGICAL SURVEY}

Statement of Water Analysis

Source We11 J34-9

Location Baxterville, Lamar Co., Mississippi

SW $1 / 4$ NW $1 / 4 \ldots 1 / 4$ Sec. 34, T.2 N., R. 16 W. Field/Office No.

Date Col. 10-26-65 Time 1350 Well Type Drilled

Col. By USGS Field detns: Temp. $\left({ }^{\circ} \mathrm{F}\right) \quad 76 \quad{ }^{\mathrm{pH}}$

Sp. Cond. ( $\mu$ mhos )

Appearance

Eh

Depth (ft.) 138

Use Bom; stock

Diam. (in.) 2 Date drilled 1952

Water level (ft.) 80 below 1.s.d., 1954

Discharge A few gpm (estimated)

W. B. F. Quaternary (alluvium,

Owner $\quad$ terrace deposits, etc.) Martha Entrekin, Baxterville, Miss.

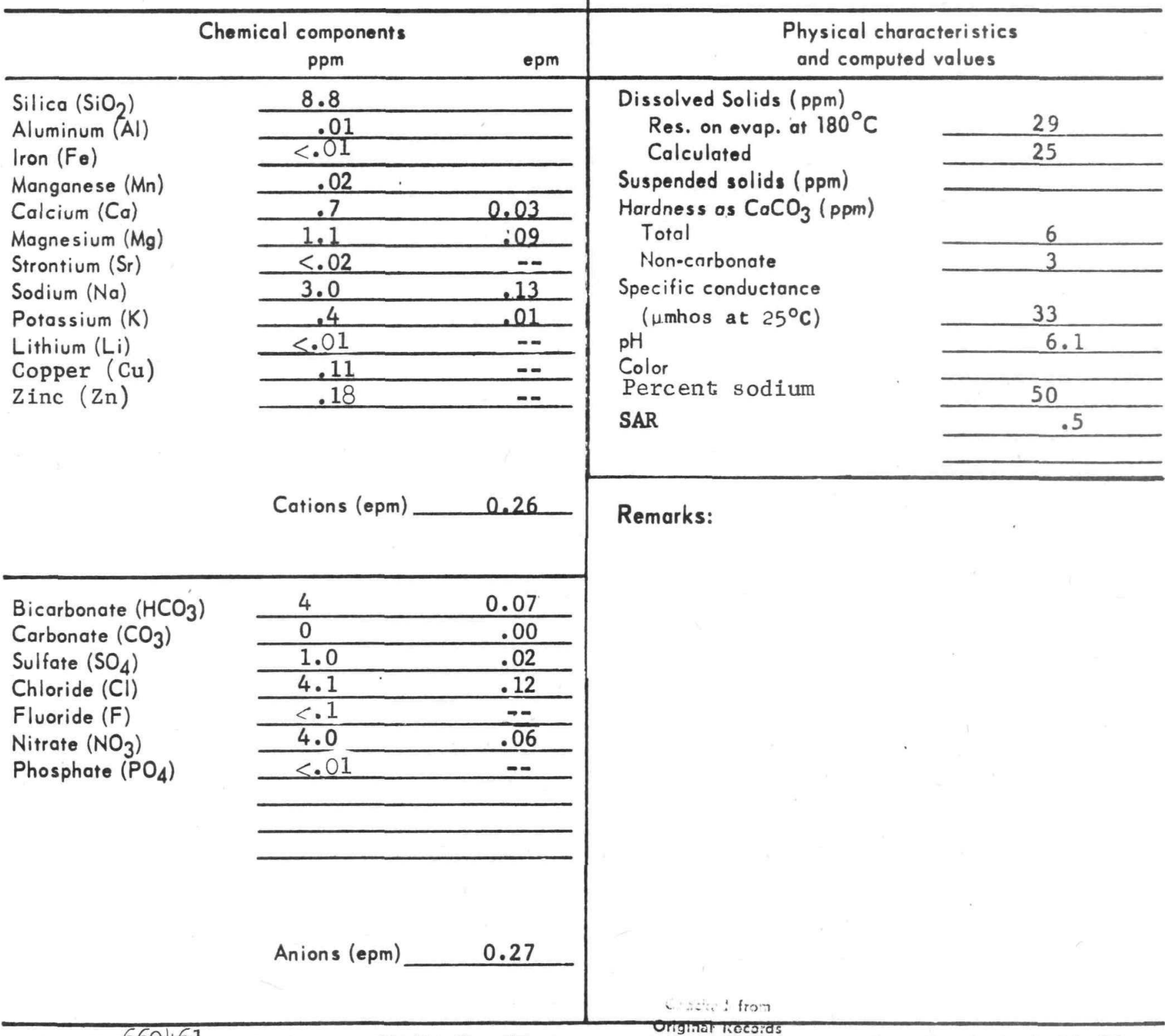

Lab. No. 660461 Analysts G. F. Scarbre 
U. S. DEPT. OF THE INTERIOR--GEOLOGICAL SURVEY

Statement of Water Analysis - Radiochemical

Source

Well J34-9

Location Baxterville, Lamar Co., Mississippi
SW
$1 / 4 \mathrm{NW} 1 / 4$
$1 / 4 \mathrm{Sec}$
4. T $2 \mathrm{~N}$.

Date Col. 10-26-65 Time 1350

Col. By USGS

Fleld detns: Temp. (OF)

Sp. Cond. ( $\mu$ mhos)

Appearance
Time 1350

76 ph

Clear
Well Type Drilled

Depth $(f t) 138$

Cased to 132

Diam. (In) 2 Date Drilled 1952

Water level (ft) 80 below 1.s.d., 1954

Discharge A few gpm (estimated)

W.B.F. Quaternary (alluvium, terrace deposits, etc.)

Owner Martha Entrekin, Baxterville, Miss.

\begin{tabular}{|c|c|c|c|c|}
\hline Elements & \multirow[t]{2}{*}{$\mathrm{pc} / 1$} & $\mathrm{pc} / \mathrm{s}$ & \multicolumn{2}{|c|}{ Date determined } \\
\hline Uranium $\mu g / 1$ & & & & \\
\hline \multicolumn{5}{|l|}{ Radlum as $\mathrm{Ra}^{2 \mathrm{~d}}$} \\
\hline Gross $\beta\left(\right.$ as $\left.\mathrm{Sr}^{90}-\mathrm{Y}^{80}\right)$ & 1.3 & & \multicolumn{2}{|c|}{$12-28-65$} \\
\hline Gross $\alpha$ as U equivalent $\mu g / 1$ & .6 & & \multicolumn{2}{|c|}{$12-28-65$} \\
\hline \multicolumn{5}{|l|}{ Net extr. $\alpha, U$ equivalent $\mu g / 1$} \\
\hline \multicolumn{5}{|l|}{ Gross Y (as $\left.\mathrm{Cs}^{137}\right)$} \\
\hline \multicolumn{5}{|l|}{ Potassiumio } \\
\hline \multicolumn{5}{|l|}{ Strontium $90 *$} \\
\hline \multirow{2}{*}{\multicolumn{5}{|c|}{ Dissolved solids $\mathrm{mg} / 1$}} \\
\hline & & & & \\
\hline \multicolumn{5}{|l|}{20} \\
\hline Isotopic Analysis & & & \multicolumn{2}{|c|}{\begin{tabular}{l|c} 
Method & $\begin{array}{c}\text { Corrected for } \\
\text { decay to: }\end{array}$
\end{tabular}} \\
\hline \multicolumn{5}{|l|}{ Barium-lanthanum ${ }^{1} 40$} \\
\hline \multicolumn{5}{|l|}{ Cerium-praseodymium ${ }^{146}$} \\
\hline \multicolumn{5}{|l|}{ Cesium ${ }^{137}$} \\
\hline \multicolumn{5}{|l|}{ Chromium ${ }^{5}$} \\
\hline \multicolumn{5}{|l|}{ Cobalt } \\
\hline \multicolumn{5}{|l|}{ Ruthenium-rhodium ${ }^{106}$} \\
\hline Tritium $\quad(\mathrm{c} / \mathrm{ml} 2-9-66)$ & $\overline{\mathrm{ND}}$ & & & \\
\hline \multicolumn{5}{|l|}{ Zinc ${ }^{65}$} \\
\hline Zirconium-niobium $^{95}$ & & & & \\
\hline & & & & \\
\hline $\begin{array}{l}\text { ND: Sought but not detected by } \\
\text { method used. }\end{array}$ & & & & \\
\hline
\end{tabular}

Date sample received 12-14-65

Date data released $1-5-66$

Program classification

*Analyzed only when Indicated by gross $\beta$ count. 58

Revised form 3-17-65
Analyst T.Curran; P.K.Roscio; H.C.Claasse Date checked 1-5-66 Checked by V.J. Janzer 


\section{U. S. DEPT. OF THE INTERIOR -- GEOLOGICAL SURVEY \\ Statement of Water Analysis}

Source We11 $\mathrm{J} 34-14$

Location Baxterville, Lamar Co., Mississippi

SW $1 / 4$ SW $1 / 4$ SE $1 / 4$ Sec. 34, T -2 N, R. 16 W. Field/Office No.

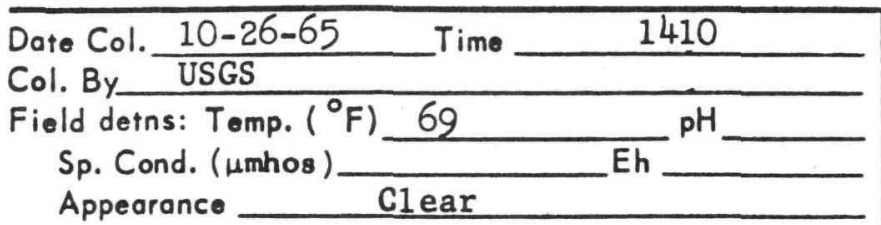

Appearance

Clear

Well Type Drilled

Depth $(f t)$.

Diam. (in.) 2

Use Dom

Cased to $94 \mathrm{ft}$.

Water level (ft.) 65 below 1.s.d., 1963

Discharge $\quad 3.5 \mathrm{gpm}, 4-25-63$ (measured)

W. B.F. Citronelle Fm.

Owner

H. G. Thompson, Baxterville, Miss.

Physical characteristics and computed values

\section{Chemical components}

Silica $\left(\mathrm{SiO}_{2}\right)$

Aluminum (Al)

Iron (Fe)

Manganese $(\mathrm{Mn})$

Calcium ( $\mathrm{Ca}$ )

Magnesium (Mg)

Strontium ( $\mathrm{Sr}$ )

Sodium ( $\mathrm{Na}$ )

Potassium (K)

Lithium (Li)

Copper ( Cu)

Zinc ( $\mathrm{Zn}$ )

\begin{tabular}{cc} 
ppm & epm \\
\hline 11 & \\
\hline$<.01$ & \\
\hline$<.01$ & \\
\hline .03 & 0.02 \\
\hline .4 & .03 \\
\hline .4 & -- \\
\hline .04 & .12 \\
\hline 2.7 & .02 \\
\hline .7 & -- \\
\hline$<.01$ & -- \\
\hline .07 & -- \\
\hline .10 &
\end{tabular}

Cations (epm)

0.19

Bicarbonate $\left(\mathrm{HCO}_{3}\right)$

Corbonate $\left(\mathrm{CO}_{3}\right)$

Sulfate $\left(\mathrm{SO}_{4}\right)$

Chloride $(\mathrm{Cl})$

Fluoride (F)

Nitrate $\left(\mathrm{NO}_{3}\right)$

Phosphate $\left(\mathrm{PO}_{4}\right)$
Dissolved Solids (ppm)

Res. on evap. af $180^{\circ} \mathrm{C}$

Calculated

Suspended solids (ppm)

Hardness os $\mathrm{CaCO}_{3}$ (ppm)

Total

Non-earbonate

Specific conductance

(umhos at $25^{\circ} \mathrm{C}$ )

$\mathrm{pH}$

Color

Percent sodium

SAR

$\begin{array}{r}26 \\ \hline 22 \\ \hline \\ 3 \\ \hline 0\end{array}$

19

6.0

63

\section{Remarks:}

\begin{tabular}{cc}
5 & 0.08 \\
\hline 0 & .00 \\
\hline 1.0 & .02 \\
\hline 2.6 & .07 \\
\hline$<.1$ & -- \\
\hline 1.5 & .02 \\
\hline$<.01$ & -- \\
\hline
\end{tabular}

Anions (epm) 0.19

Lab. No. 660462 Analysts_ G. F: Scarbro 
U. S. DEPT. OF THE INIERIOR--GEOLOGICAI SURVEY

Statement of Water Analysis - Radiochemical

Source

Wel1 J34-14

Location Baxterville, Lamar Co., Mississippi SW $1 / 4$ SW $1 / 4$ SE $1 / 4 \mathrm{Sec} 34, \quad \mathrm{~T} 2 \mathrm{~N} .1$ R 16 W. Field/Office No.

Date Col. 10-26-65 Time 1410

Col. By USGS

Field detns: Temp. (OF) Sp. Cond. ( $\mu$ mhos) Appearance

$69 \mathrm{pH}$
Eh

Clear

Elements

Uranium

Radium as $\mathrm{Ra}^{225}$

Gross $\beta\left(\right.$ as $\mathrm{Sr}^{90}$ - $\left.\mathrm{Y}^{00}\right)$

Gross $\alpha$ as U equivalent $\mu \mathrm{g} / 1$

Net extr. $\alpha$, U equivalent $\mu g / 1$

Gross $Y\left(\right.$ as $\left.\mathrm{Cs}^{137}\right)$

Potassium 40

Strontium 90 *

Dissolved solids mg/1

Isotopic Analysis

Barium-lanthanum 140

Cerium-praseodymium ${ }^{46}$

Cesium³7

Chromium57

Cobalto

Ruthenium-rhodium 106

Tritium (uc/m1 2-9-66)

Zinc $^{65}$

Zirconium-niobium ${ }^{95}$

ND: Sought but not detected by

method used

Date sample received 12-14-65

Date data released $1-5-66$

Program classification

*Analyzed only when indicated by gross $\beta$ count.

Revised form $3-17-65$

Well Type Drilled Use Dom

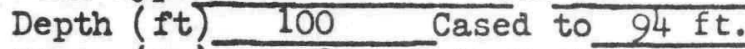

Diam. (In) Date Drilled March 1961

Water level (ft) 65 below 1.s.d., 1963

Discharge $3.5 \mathrm{gpm}, 4-25-63$ (measured)

W.B.F. Citronelle Fm.

Owner H. G. Thompson,

Baxterville, Miss.

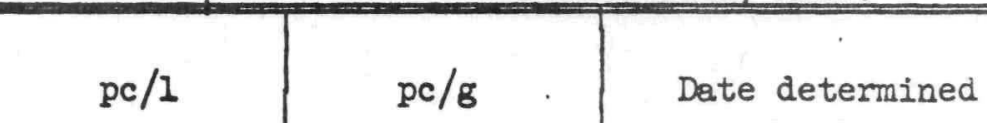




\section{U. S. DEPT. OF THE INTERIOR -- GEOLOGICAL SURVEY \\ Statement of Water Analysis}

Source We11 J36-4

Location Baxterville, Lamar Co., Mississippi

NW $1 / 4$ SE $1 / 4 \ldots 1 / 4$ Sec. $36, \quad T, 2$ N., R. 16 W. Field/Office No.

Date Col. 10-26-65 Time 1440

Col. By USGS

Field detns: Temp. $\left({ }^{\circ} \mathrm{F}\right)$

Sp. Cond. ( $\mu$ mhos )

Appearance

68

Clear
$\mathrm{pH}$

Eh

-

(


U. S. DEPT. OF THE INTERIOR--GEOLOGICAL SURVEY

Statement of Water Analysis - Radiochemical

Source Well J36-4

Location Baxterville, Lamar Co., Mississippi

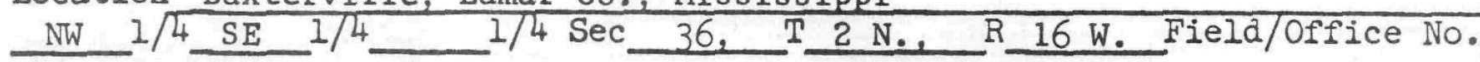

\begin{tabular}{|c|c|}
\hline Date Col. $10-26-65$ & Use Dom; stoek \\
\hline Col. By & Depth $(f t)$ \\
\hline $\begin{array}{l}\text { Field detns: Temp. (OF) } \\
\text { Sp. Cond. ( } \mu \text { mhos) }\end{array}$ & 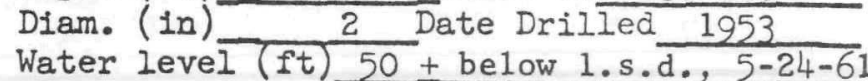 \\
\hline Appearance & $\begin{array}{ll}\text { Discharge } & \frac{5.4 \mathrm{gpm}, 1963(?)}{\text { Quaternary (alluvium, }} \\
\text { W.B.F. }\end{array}$ \\
\hline & terrace deposits, etc.) \\
\hline & $\begin{array}{l}\text { Arthur Lowe, } \\
\text { Baxterville, Miss. }\end{array}$ \\
\hline
\end{tabular}

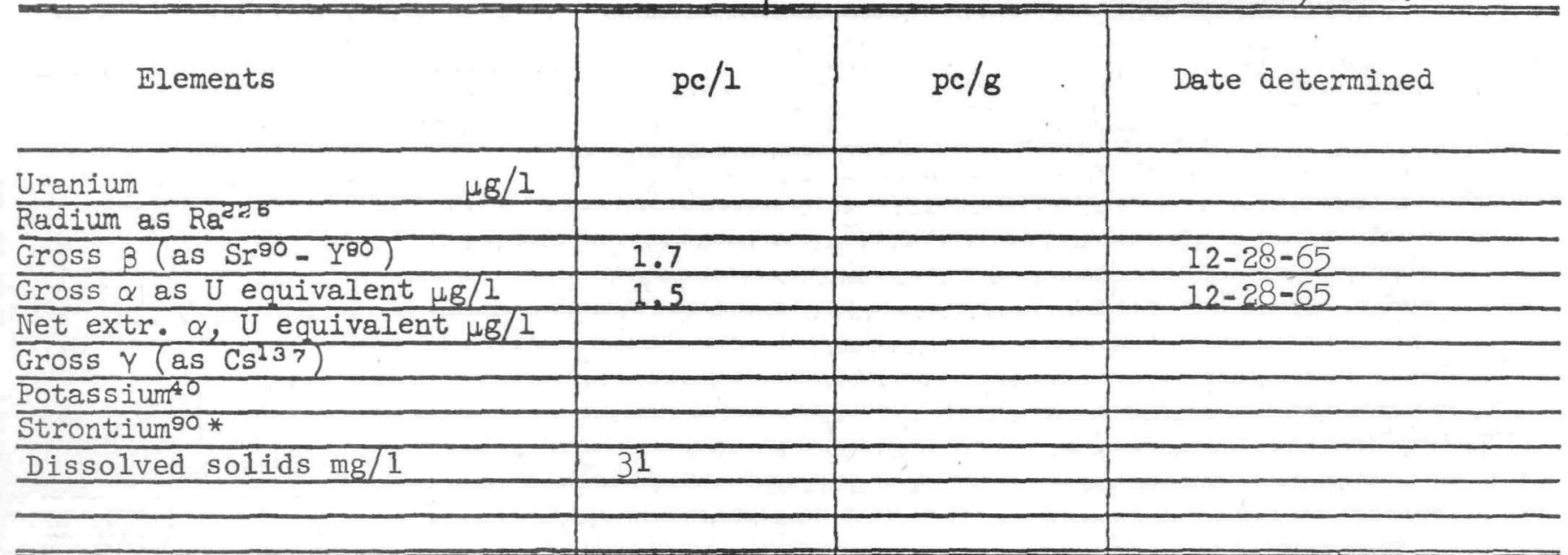

Isotopic Analysis

Barium-Ianthanum 140

Cerium-praseodymium ${ }^{46}$

Cesium ${ }^{37}$

Chromium ${ }^{57}$

Cobalt50

Ruthenium-rhodium 106

Tritium

Zinc ${ }^{65}$

Zirconium-niobium ${ }^{95}$

ND: Sought but not detected by

method used.

Date sample received 12-14-65

Date data released $1-5-66$

Program classification

\begin{tabular}{l|l|l|l} 
& & Method & $\begin{array}{c}\text { Corrected for } \\
\text { decay to: }\end{array}$ \\
\hline & & & \\
\hline & & & \\
\hline ND & & & \\
\hline & & & \\
\hline & & & \\
\hline
\end{tabular}

*Analyzed only when Indicated by gross a count.

Revised form 3-17-65

62

Analyst T.Curran; P.K.Roscio; H.C.Claassen Date checked 1-5-66

Checked by V.J.Janzer

Checked from

Original Records

Bate $2-22-66$ 


\section{U. S. DEPT. OF THE INTERIOR .. GEOLOGICAL SURVEY \\ Statement of Water Analysis}

Source

We11 K7-1

Location Lumberton, Lamar Co., Mississippi

NW $1 / 4$ SW $1 / 4$ $1 / 4$ Sec. 7 , T:-

Date Col. 10-26-65 Time 1615

Col. By USGS

Field detns: Temp. $\left({ }^{\circ} \mathrm{F}\right)$

Sp. Cond. ( $\mu$ mhos)

Appearance

68

Colored

$\mathrm{pH}$

H

-

\begin{tabular}{|c|c|c|}
\hline \multicolumn{3}{|c|}{ Chemical components } \\
\hline & ppm & epm \\
\hline Silica $\left(\mathrm{SiO}_{2}\right)$ & 8.0 & \\
\hline Aluminum (Al) & $<.01$ & \\
\hline Iron $\left(F_{e}\right)$ & $<.01$ & \\
\hline Manganese (Mn) & .07 & \\
\hline Calcium (Ca) & .3 & 0.01 \\
\hline Magnesium (Mg) & .5 & .04 \\
\hline Strontium (Sr) & $<.02$ & -- \\
\hline Sodium ( $\mathrm{Na}$ ) & 2.8 & .12 \\
\hline Potassium (K) & .6 & .02 \\
\hline Lithium (Li) & $<.01$ & $=$ \\
\hline Copper ( $\mathrm{Cu})$ & .12 & - \\
\hline Zinc $(2 n)$ & .31 & -- \\
\hline
\end{tabular}
$15 \mathrm{~W}$. Field/Office No.

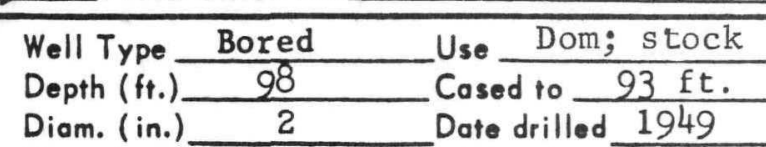

Water level (ft.) 76 below 1.s.d. 1949

Discharge $4 \mathrm{gpm}, 3-27-63$ (measured)

W. B. F. Quaternary (alluvium, terrace deposits, etc.)

Owner R. L. Anderson, Sr., Rt. 4, Lumberton, Miss.

\section{Physical characteristics} and computed values

Dissolved Solids (ppm)

Res. on evap. of $180^{\circ} \mathrm{C}$

Calculated

Suspended solids (ppm)

Hardness as $\mathrm{CaCO}_{3}$ (ppm)

Total

Non-carbonate

Specific conductance

(umhos at $25^{\circ} \mathrm{C}$ )

$\mathrm{pH}$

Color

Percent sodium

SAR

$\begin{array}{r}22 \\ \hline 20 \\ \hline 3 \\ \hline 0 \\ \hline\end{array}$

21

6.0

$\begin{array}{r}\frac{21}{6.0} \\ \hline-63 \\ \hline .8 \\ \hline\end{array}$

Cations (epm) 0.19 Remarks:

Bicarbonate $\left(\mathrm{HCO}_{3}\right)$

Carbonate $\left(\mathrm{CO}_{3}\right)$

Sulfate $\left(\mathrm{SO}_{4}\right)$

Chloride (Cl)

Fluoride ( $F$ )

Nitrate $\left(\mathrm{NO}_{3}\right)$

Phosphate $\left(\mathrm{PO}_{4}\right)$

\begin{tabular}{lc}
\hline 4 & 0.07 \\
\hline 0 & .00 \\
\hline 1.0 & .02 \\
\hline 2.4 & .07 \\
\hline$<.1$ & -- \\
\hline 2.2 & .04 \\
\hline$<.01$ & -- \\
\hline
\end{tabular}

Anions (epm) $\quad 0.20$ 
U. S. DEPT. OF THE INTERIOR--GEOLOGICAL SURVEY

Statement of Water Analysis - Radiochemical

Source

Location Lumberton, Lamar Co., Mississippi

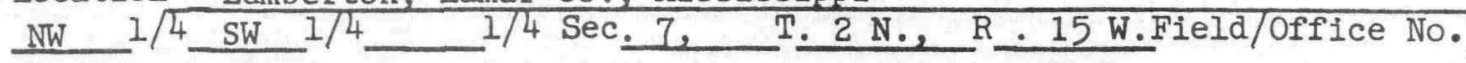

Date Col. 10-26-65 Time_ 1615

Col. By USGS Depth (ft 98 Cased to $93 \mathrm{ft}$.

- Field detns: Temp. (OF) $68 \quad$ pH_ Diam. (in) 2 Date Drilled 1949

$\mathrm{Sp}$. Cond. ( $\mu$ mhos )

Appearance

Eh

Colored
Water level (ft) 76 below 1.s.d., 1949

Discharge 4 gpm, 3-27-63 (measured)

W.B.F. Quaternary (alluvium, terrace deposits, etc.)

Owner R. L. Anderson, Sr., Rt. 4, Lumberton, Miss.

\begin{tabular}{|c|c|c|c|c|}
\hline Elements & $\mathrm{pc} / \mathrm{l}$ & $\mathrm{pc} / \mathrm{g}$ & \multicolumn{2}{|c|}{ Date determined } \\
\hline \multicolumn{5}{|l|}{ Uranium $\mu g / 1$} \\
\hline \multicolumn{5}{|l|}{ Radium as $\mathrm{Ra}^{\mathrm{L} \mathrm{C}_{\mathrm{O}}}$} \\
\hline Gross $\beta\left(\right.$ as Sr90 $\left.-Y^{90}\right)$ & 3.8 & & \multicolumn{2}{|c|}{$2-5-66$} \\
\hline Gross $\alpha$ as U equivalent $\mu g / 1$ & 1.1 & & \multirow{2}{*}{\multicolumn{2}{|c|}{$2-5-66$}} \\
\hline \multicolumn{3}{|l|}{ Net extr. $\alpha$, U equivalent $\mu g / 1$} & & \\
\hline \multicolumn{5}{|l|}{ Gross $\gamma\left(\operatorname{as~} \mathrm{Cs}^{137}\right)$} \\
\hline \multicolumn{5}{|l|}{ Potassium ${ }^{40}$} \\
\hline \multicolumn{5}{|l|}{ Strontium $90 *$} \\
\hline \multirow{2}{*}{\multicolumn{5}{|c|}{ Dissolved solids $\mathrm{mg} / 1$}} \\
\hline & & & & \\
\hline \multicolumn{5}{|l|}{ (20) } \\
\hline Isotopic Analysis & & & Method & $\begin{array}{l}\text { Corrected for } \\
\text { decay to: }\end{array}$ \\
\hline \multicolumn{5}{|l|}{ Barium-lanthanum 140} \\
\hline \multicolumn{5}{|l|}{ Cerium-praseodymium ${ }^{146}$} \\
\hline \multicolumn{5}{|l|}{ Cesium ${ }^{137}$} \\
\hline \multicolumn{5}{|l|}{ Chromium ${ }^{7}$} \\
\hline \multicolumn{5}{|l|}{ Cobalt } \\
\hline \multicolumn{5}{|l|}{ Ruthenium-rhodium ${ }^{106}$} \\
\hline$(\mu \mathrm{c} / \mathrm{ml} \mathrm{2-9-66)}$ & $\mathrm{ND}$ & & & \\
\hline \multicolumn{5}{|l|}{$\operatorname{Zinc}^{65}$} \\
\hline \multicolumn{5}{|l|}{ Zirconium-niobium ${ }^{95}$} \\
\hline ND: Sought but not detected & & & & \\
\hline used. & & & & \\
\hline
\end{tabular}

Date sample received 12-14-65

Date data released 2-11-66

Program classification

*Analyzed only when indicated by gross $\beta$ count.

Revised form 3-17-65
Analyst P.K.Roscio: T.Curran: H.C.Claassep Date checked 2-11-66

Checked by V.J. Janzer
$64 \quad \begin{gathered}\text { Checked from } \\ \text { Original Records }\end{gathered}$

$6 P O 848 \cdot 788$ 


\section{U. S. DEPT. OF THE INTERIOR -. GEOLOGICAL SURVEY \\ Statement of Water Analysis}

Source

We11 K7-4

Location Purvis, Lamar Co, Mississippi

SE $1 / 4$ SW $1 / 4 \ldots$ Sec. 7, T. 2 N., R. 15 W. Field/Office No.

\begin{tabular}{ll|ll}
\hline Date Col. $\frac{10-26-65}{\text { USGS }}$ & Time & $\begin{array}{l}\text { Well Type } \\
\text { Depth (ft.) Bored }\end{array}$ \\
Col. By & De Use Dom; stock
\end{tabular}

Field derns: Temp. $\left({ }^{\circ} \mathrm{F}\right)$

Sp. Cond. (umhos)

Appearance
68

$\mathrm{pH}$

Eh

Clear
Diam. (in.) 6 to 2 Date drilled 1951

Water level (ft.) 70 below 1.s.d., 1961

Discharge $3 \mathrm{gpm}, 8-18-64$

W. B. F. Quaternary (alluvium, terrace, deposits, etc.

Owner G. W. Anderson. Rt. 1, Purvis, Miss.

\begin{tabular}{|c|c|c|}
\hline \multicolumn{3}{|c|}{ Chemical components } \\
\hline & ppm & epm \\
\hline Silica $\left(\mathrm{SiO}_{2}\right)$ & 8.0 & \\
\hline Aluminum (Al) & $<.01$ & \\
\hline Iron $(\mathrm{Fe})$ & $<.01$ & \\
\hline Manganese $\left(\mathrm{Mn}_{\mathrm{n}}\right)$ & .10 & \\
\hline Calcium (Ca) & .6 & 0.03 \\
\hline Magnesium (Mg) & .4 & .03 \\
\hline Strontium (Sr) & .15 & -- \\
\hline Sodium (Na) & 1.5 & .07 \\
\hline Potossium (K) & .6 & .02 \\
\hline Lithium (Li) & .01 & -- \\
\hline Copper (Cu) & .03 & -- \\
\hline Zinc $(\mathrm{Zn})$ & .17 & -- \\
\hline
\end{tabular}

Cations (epm) 0.15

Physical characteristics and computed values

Dissolved Solids (ppm)

Res. on evap. at $180^{\circ} \mathrm{C}$

Calculated

Suspended solids (ppm)

Hardness as $\mathrm{CaCO}_{3}(\mathrm{ppm})$

Total

Non-carbonate

Specific conductance

( $\mu$ mhos at $25^{\circ} \mathrm{C}$ )

$\mathrm{pH}$

Color

Percent sodium

SAR

$\begin{array}{r}16 \\ \hline 17 \\ \hline \\ \hline 3 \\ \hline 0\end{array}$

$\frac{16}{6.0}$

\section{Remarks:}

\begin{tabular}{cc}
4 & 0.07 \\
\hline 0 & .00 \\
\hline$<.1$ & -- \\
\hline 2.4 & .07 \\
\hline$<.1$ & .00 \\
\hline 1.2 & .02 \\
\hline .11 & -- \\
\hline
\end{tabular}

Bicarbonate $\left(\mathrm{HCO}_{3}\right)$

Carbonate $\left(\mathrm{CO}_{3}\right)$

Sulfate $\left(\mathrm{SO}_{4}\right)$

Chloride $(\mathrm{Cl})$

Fluoride ( $F$ )

Nitrate $\left(\mathrm{NO}_{3}\right)$

Phosphate $\left(\mathrm{PO}_{4}\right)$

$\overline{-}$

Anions (epm) 0.16 
U. S. DEPT. OF THE INTERIOR--GEOLOGICAL SURVEY

Statement of Water Analysis - Radiochemital

Source

Well K7-4

Location Purvis, Lamar Co., Mississippi

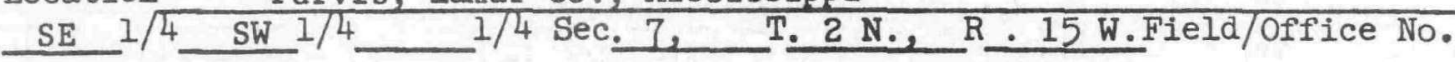

Date Col. 10-26-65 Time $1610 \quad$ Well Type Bored Use Dom; stock

Col. By USGS Depth $(\mathrm { ft } \longdiv { 8 0 }$

Cased to 80

Field detns: Temp. (oF) 68 pH

Sp. Cond. ( $\mu$ mhos

Appearance Clear

Diam. (in) 6 to 2 Date Drilled 1951

Water level (ft) 70 below 1.s.d., 1961

Discharge

W.B.F. Quaternary (alluvium. 3 gpm, 8-18-64

Owner_G. W. Anderson,

osits, etc.)

Rt. 1, Purvis, Miss.

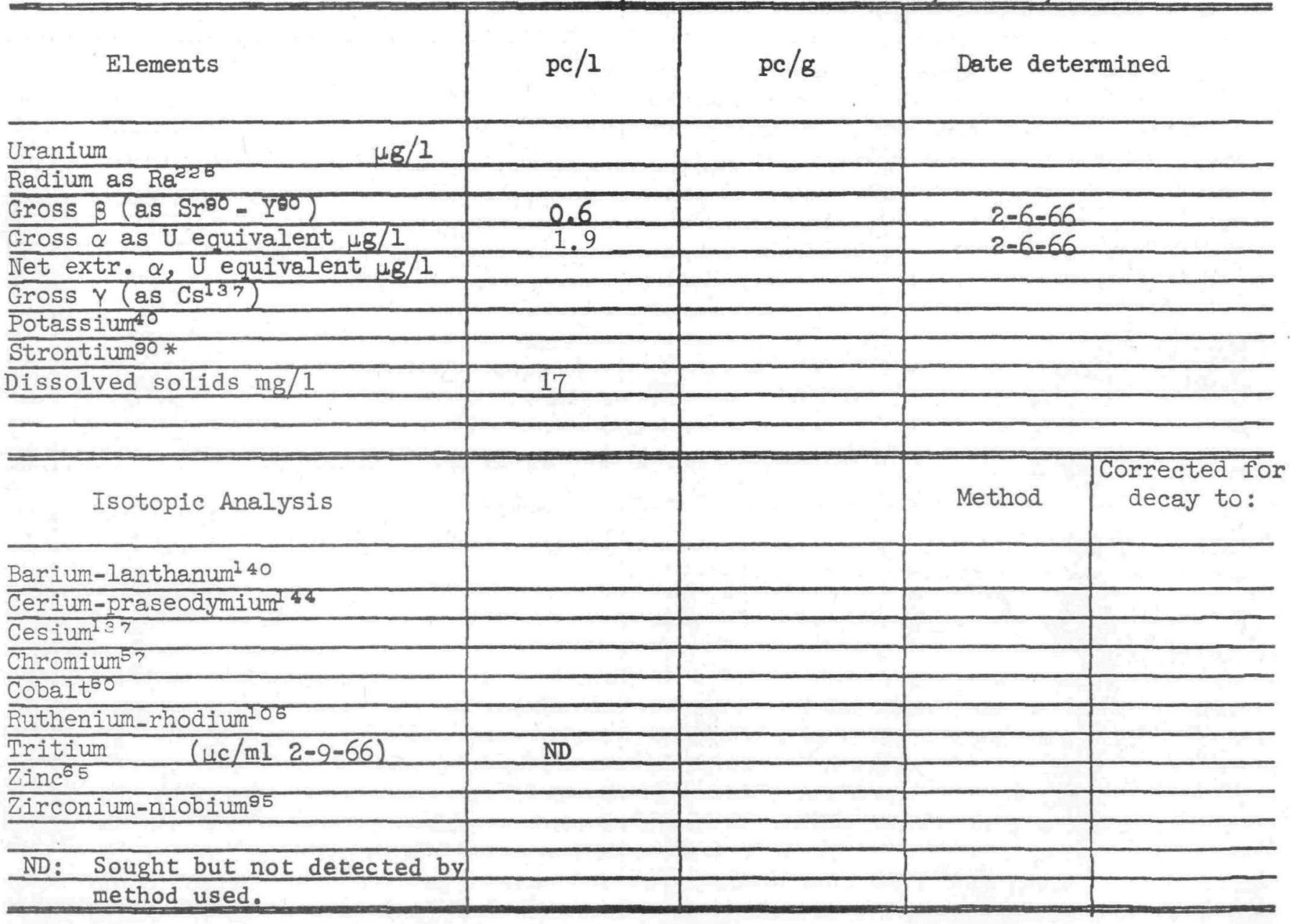

Date sample received 12-14-65

Date data released 2-11-66

Program classification

*Ans.lyzed only when indicated by gross $\beta$ count.

Revised form 3-17-65
Analyst P.K.Roscio: E.Villasana:H.C.Claasser Date checked 2-11-66 Checked by V.J. Janzer 


\section{U. S. DEPT. OF THE INTERIOR .. GEOLOGICAL SURVEY Statement of Water Analysis}

Source

We11 K9-2

Location Purvis, Lamar Co., Mississippi

$\mathrm{SE} 1 / 4 \mathrm{SE} 1 / 4 \ldots 1 / 4$ Sec. 2, T. 2 N., R. $15 \mathrm{~W}$, Field/Office No.

Date Col. 10-25-65 Timo 1645

Col. By USGS

Field detns: Tomp. $\left({ }^{\circ} \mathrm{F}\right)$

Sp. Cond. (umhos)

Appearance

69

$\mathrm{PH}^{\mathrm{pH}}$

$\mathrm{pH}$

Clear
Well Type Drilled

Depth (ft.) 131

Diam. (in.) 2

Use Dom; stock

Cased to $125 \mathrm{ft}$.

Water level (ft.) 80 below 1.s.d. 1959

Discharge A few gpm (estimated)

W. B. F. Quaternary (alluvium, terrace deposits, etc.)

Owner Mississippi Forestry Commission Rt. 1, Purvis, Miss.

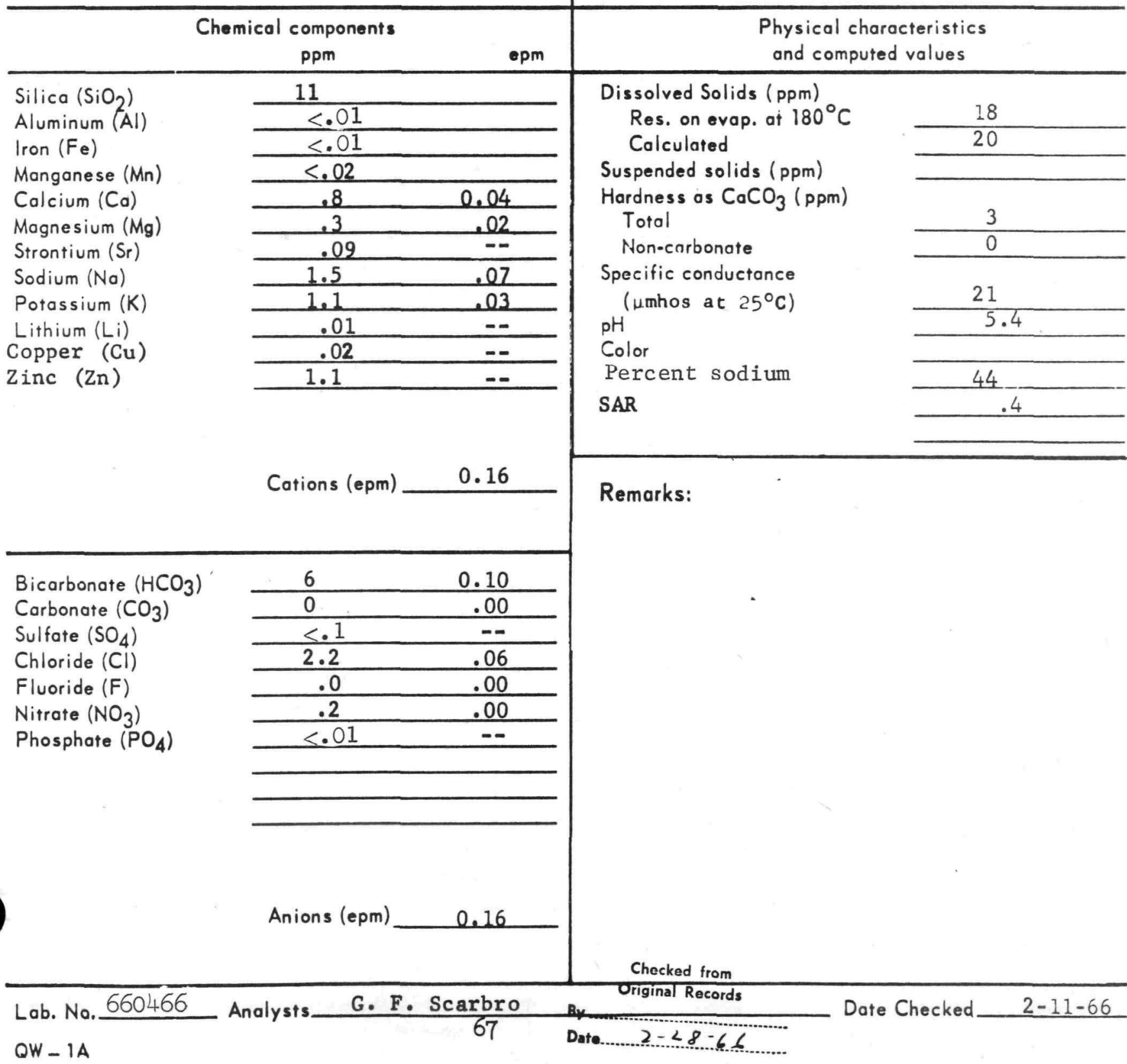


Lab. No.

U. S. DEPT. OF THE INTERIOR--GEOLOGICAL SURVEY

Statement of Water Analysis - Radiochemital

Source

Well K9-2

Location Purvis, Lamar Co.. Mississippi

$\mathrm{SE} 1 / 4 \mathrm{SE} 1 / 4 \quad 1 / 4 \mathrm{Sec} 9 \mathrm{~T}$

2.N. R 15 W. Field/Office No.

Date Col. 10-25-65 Time 1645

Col. By USGS

1645

Field detns: Temp. (OF)

Sp. Cond. ( $\mu$ mhos)

\section{Sp. Cond.
Appearance}

$\frac{69-\frac{\mathrm{pH}}{\mathrm{Eh}}}{\mathrm{Clear}}$

Well Type Drilled

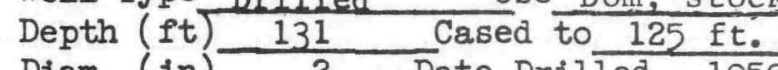

Diam. (in) $\frac{2}{2}$ Date Drilled 1959

Water level(ft) 80 below 1.s.d. 1959

Discharge

W.B.F.
A few gpm (estimated)

Quaternary (alluvium.

terrace deposits, etc.)

Owner Mississippi Forestry Commission

Rt. 1, Purvis, Miss.

\begin{tabular}{|c|c|c|c|c|}
\hline Elements & \multirow[t]{2}{*}{$\mathrm{pc} / 1$} & \multirow[t]{2}{*}{$\mathrm{pc} / \mathrm{s}$} & \multicolumn{2}{|c|}{ Date determined } \\
\hline \multirow{2}{*}{\multicolumn{5}{|c|}{ 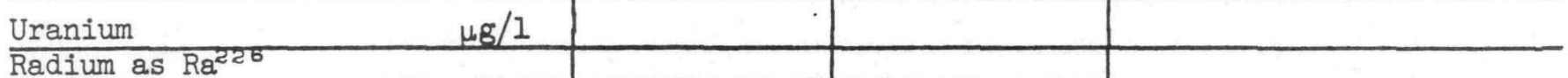 }} \\
\hline & & & & \\
\hline Gross $\beta\left(\right.$ as $\left.\mathrm{Sr}^{90}-\mathrm{Y}^{80}\right)$ & 1.1 & & \multirow{2}{*}{\multicolumn{2}{|c|}{$\frac{1-8-66}{1-8-66}$}} \\
\hline Gross $\alpha$ as U equivalent $\mu \mathrm{g} / \mathrm{I}$ & 1.1 & & & $1-8-66$ \\
\hline \multicolumn{5}{|l|}{ Net extr. $\alpha, \mathrm{U}$ equivalent $\mu \mathrm{g} / \mathrm{l}$} \\
\hline \multicolumn{5}{|l|}{ Gross Y (as $\left.\operatorname{Cs}^{137}\right)$} \\
\hline \multicolumn{5}{|l|}{ Potassium 40} \\
\hline \multicolumn{5}{|l|}{ Strontium90* } \\
\hline \multicolumn{5}{|l|}{ Dissolved solids mg/1 } \\
\hline \\
\hline Isotopic Analysis & & & Method & $\begin{array}{l}\text { Corrected for } \\
\text { decay to: }\end{array}$ \\
\hline \multicolumn{5}{|l|}{ Barium-lanthanum ${ }^{1} 40$} \\
\hline \multicolumn{5}{|l|}{ Cerium-praseodymium ${ }^{146}$} \\
\hline \multicolumn{5}{|l|}{ Cesium ${ }^{137}$} \\
\hline \multicolumn{5}{|l|}{ Chromium ${ }^{7}$} \\
\hline \multicolumn{5}{|l|}{ Cobalt } \\
\hline \multicolumn{5}{|l|}{ Ruthenium_rhodium ${ }^{106}$} \\
\hline$(\mu \mathrm{c} / \mathrm{ml} \quad 2-9-66)$ & $\mathrm{ND}$ & & & \\
\hline \multicolumn{5}{|l|}{ Zinc ${ }^{65}$} \\
\hline \multicolumn{5}{|l|}{ Zirconium-niobium $^{95}$} \\
\hline ND: Sought but not detected by & & & & \\
\hline method used. & & & & \\
\hline
\end{tabular}

Date sample received 12-14-65

Date data released 1-17-66

Program classification

*Analyzed only when indicated by gross $\beta$ count.

Revised form 3-17-65
68
Analyst T.Curran; E.Villasana;H.C.Claassen Date checked $1-17-66$

Checked by V.J. Janzer 


\section{U. S. DEPT. OF THE INTERIOR -- GEOLOGICAL SURVEY \\ Statement of Water Analysis}

Source We11 K18-8

Location Lumberton, Lamar Co., Mississippi

NW $1 / 4$ SW $1 / 4 \ldots 1 / 4$ Sec. $18, T \cdot 2$ N., R. 15 W. Field/Office No.

Date Col. $10-26-65$ Time 1555

Col. By USGS

Field detns: Tomp. $\left({ }^{\circ} \mathrm{F}\right) \quad 68$

Sp. Cond. (umhos)

Appearance

$\mathrm{pH}$

Clear

Well Type Drilled

Depth (ft.) 78

Diam. (in.) 2

Water level (ft.) Unknown

Discharge A few gpm (estimated)

W. B. F. Quaternary (alluvium, terrace deposits, etc.)

Owner R. L. Anderson, $\mathrm{Jr}$. Rt. 4, Lumberton, Miss.

\begin{tabular}{|c|c|c|}
\hline \multicolumn{3}{|c|}{ Chemical components } \\
\hline Silica $\left(\mathrm{SiO}_{2}\right)$ & 8.0 & \\
\hline Aluminum (Al) & $<.01$ & \\
\hline Iron $(F e)$ & $<.01$ & \\
\hline Manganese $(M n)$ & .06 & \\
\hline Calcium (Ca) & 1.4 & 0.07 \\
\hline Magnesium (Mg) & 1.0 & .08 \\
\hline Strontium (Sr) & .08 & -- \\
\hline Sodium $(\mathrm{Na})$ & 1.6 & .07 \\
\hline Potassium (K) & .6 & .02 \\
\hline Lithium (Li) & .01 & $=-$ \\
\hline Copper (Cu) & .02 & - \\
\hline Zinc $(\mathrm{Zn})$ & 2.7 & -- \\
\hline
\end{tabular}

\section{Physical characteristics and computed values}

Disssolved Solids (ppm)

Res. on evap. at $180^{\circ} \mathrm{C}$

Calculated

Suspended solids (ppm)

Hardness os $\mathrm{CaCO}_{3}$ (ppm)

Total

Non-carbonate

Specific conductance

(u.mhos at $25^{\circ} \mathrm{C}$ )

$\mathrm{pH}$

Color

Percent sodium

SAR

$\begin{array}{r}20 \\ \hline 22 \\ \hline \\ \hline 8 \\ \hline 1 \\ \hline 26 \\ \hline 5.6 \\ \hline 29 \\ \hline .3\end{array}$

Cations (epm) $0.24 \quad$ Remarks:

Bicarbonate $\left(\mathrm{HCO}_{3}\right)$

Carbonate $\left(\mathrm{CO}_{3}\right)$

Sulfate $\left(\mathrm{SO}_{4}\right)$

Chloride (Cl)

Fluoride (F)

Nitrate $\left(\mathrm{NO}_{3}\right)$

Phosphate $\left(\mathrm{PO}_{4}\right)$

\begin{tabular}{cc}
8 & 0.13 \\
\hline 0 & .00 \\
\hline$<.1$ & -- \\
\hline 2.4 & .07 \\
\hline$<.1$ & -- \\
\hline 2.9 & .05 \\
\hline$<.01$ & -- \\
\hline
\end{tabular}

Anions (epm)

0.25 
U. S. DEPT. OF THE INIERIOR--GEOLOGICAL SURVEY

Statement of Water Analysis - Radiochemical

Source

We11 K-18-8

Location Lumberton, Lamar Co., Mississippi

$\mathrm{NW} 1 / 4 \mathrm{SW} 1 / 4 \quad 1 / 4 \mathrm{Sec}_{0} 18$, T. 2 N., R. 15 W. Field/Office No.

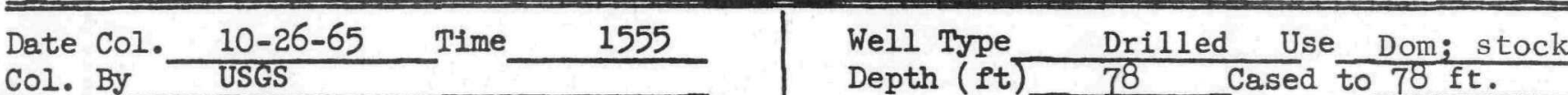

Col. By deld

Sp. Cond. ( $\mu$ mhos )

Appearance

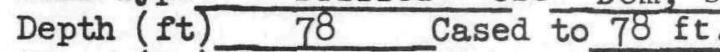

Diam. (1n) $\frac{2}{2}$ Date DrilledJuly 1963

Water level (ft) Unknown

Discharge A few gpm (estimated)

W.B.F. Quaternary (alluvium,

terrace deposits, etc.)

Owner R. L. Anderson, Jr.

Rt. 4, Lumberton, Miss.

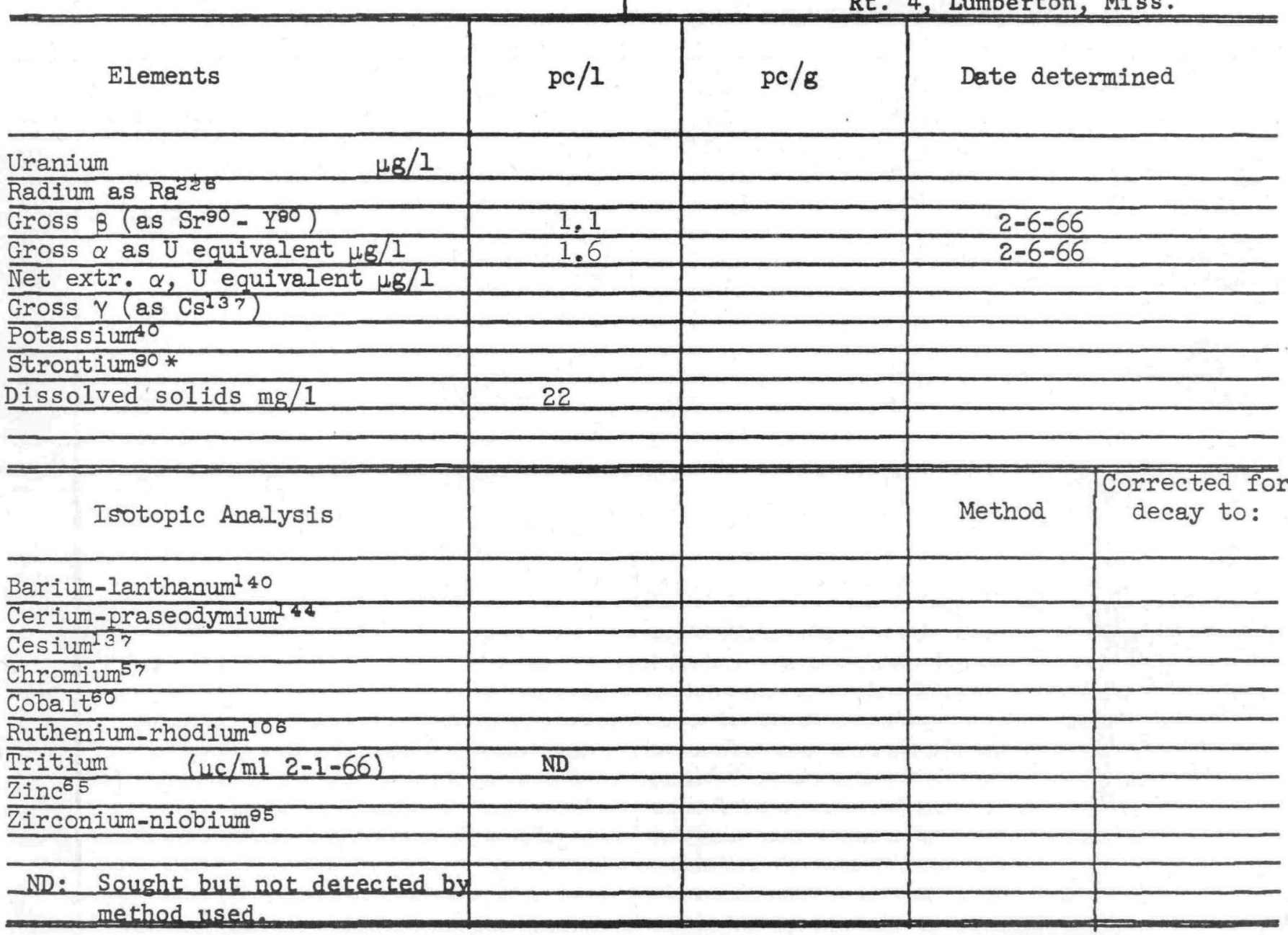

Date sample received 12-14-65

Date data released 2-11-66

Program classification
Analyst P.K.Roscio: E.Villasana;H.C.Claass Date checked 2-11-66

Checked by V. J. Janzer

*Analyzed only when indicated by gross $\beta$ count.

Revised form 3-17-65

Checked from

Original Records

C. $2-22-66$

$6 P 0848.788$ 


\section{U. S. DEPT. OF THE INTERIOR -. GEOLOGICAL SURVEY \\ Statement of Water Analysis}

Source We11 K33-2

Location Purvis, Lamar Co., Mississippi

NE $1 / 4$ NW $1 / 4 \ldots 1 / 4$ Sec. 33, T. 2 N.2, R. 15 W. Field/Office No.

Date Col. $\frac{10-26-65}{\text { USGS Time } 1510} \quad$ Well Type Bored Use Dom; stock

Field detns: Temp. $\left({ }^{\circ} \mathrm{F}\right) 67 \quad$ PH $\quad$ Diam. (in.) 8 Date drilled 1936

Sp. Cond. (umhos)

Appearance
Water lovel ( $\mathrm{ft}$. ) 30.37 below 1.s.d., 4-23-65

Discharge Bucket well

W. B. F. Quaternary (alluvium, terrace, deposits, etc.)

Owner Mrs. W. M. Courtney, Rt. 1, Purvis, Miss.

\begin{tabular}{|c|c|c|}
\hline \multicolumn{3}{|c|}{ Chemical components } \\
\hline Silica $\left(\mathrm{SiO}_{2}\right)$ & 7.3 & \\
\hline Aluminum $(\mathrm{Al})$ & $<.01$ & \\
\hline Iron $(\mathrm{Fe})$ & $<.01$ & \\
\hline Manganese $(M n)$ & .14 & \\
\hline Calcium (Ca) & 1.6 & 0.08 \\
\hline Magnesium (Mg) & .4 & .03 \\
\hline Strontium ( $\mathrm{Sr}$ ) & .06 & -- \\
\hline Sodium (Na) & 1.6 & .07 \\
\hline Potassium (K) & .5 & ـ1 \\
\hline Lithium (Li) & .02 & -- \\
\hline Copper (Cu) & .02 & -- \\
\hline Zinc $(\mathrm{Zn})$ & .19 & -- \\
\hline
\end{tabular}

Physical characteristics and computed values

Dissolved Solids (ppm)

Res. on evap. af $180^{\circ} \mathrm{C}$

Calculated

Suspended solids (ppm)

Hardness as $\mathrm{CaCO}_{3}(\mathrm{ppm})$

Total

Non-carbonate

Specific conductance

( $\mu$ mhos at $25^{\circ} \mathrm{C}$ )

$\mathrm{pH}$

Color

Percent sodium

SAR

$\begin{array}{r}20 \\ \hline 19 \\ \hline \\ 6 \\ \hline 2\end{array}$

19

5.6

37

\section{Cations (epm) $0.19 \quad$ Remarks:}

Bicarbonate $\left(\mathrm{HCO}_{3}\right)$

Carbonate $\left(\mathrm{CO}_{3}\right)$

Sulfate $\left(\mathrm{SO}_{4}\right)$

Chloride $(\mathrm{Cl})$

Fluoride (F)

Nitrate $\left(\mathrm{NO}_{3}\right)$

Phosphate $\left(\mathrm{PO}_{4}\right)$

\begin{tabular}{cc}
4 & 0.07 \\
\hline 0 & .00 \\
\hline$<.1$ & -- \\
\hline 2.6 & .07 \\
\hline$<.1$ & -- \\
\hline 2.8 & .05 \\
\hline$<.01$ & -- \\
\hline
\end{tabular}

Checked from

Original Records Analysts G. F. Searbro

$Q W-1 A$ 
U. S. DEPT. OF THE INTERIOR--GEOLOGICAL SURVEY

Statement of Water Analysis - Radiochemical

Source

We11 K33-2

Location Purvis, Lamar Co., Mississippi

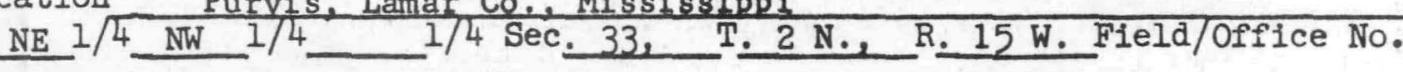

Date Col. 10-26-65 Time 1510

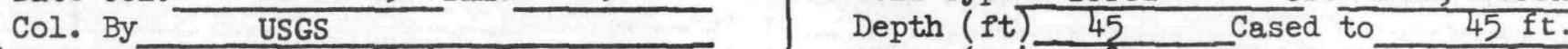

Field detns: Temp. (F) $67 \quad$ pH__ Diam. (in) 8 Date Drilled 1936

Sp. Cond. ( $\mu$ mhos) Eh - Water level (ft)30.37 below 1.s. d., 4-23-65

Appearance

Clear - Discharge

Bucket well

W.B.F. Quaternary (alluvium,

terrace deposits, etc.)

owner Mrs. W. M. Courtney,

Rt. 1, Purvis, Miss.

\begin{tabular}{|c|c|c|c|c|}
\hline Elements & $\mathrm{pc} / \mathrm{l}$ & $\mathrm{pc} / \mathrm{g}$ & \multicolumn{2}{|c|}{ Date determined } \\
\hline \multirow{2}{*}{\multicolumn{5}{|c|}{ Uranium $\quad \mu g / 1$}} \\
\hline & & & & \\
\hline Gross $\beta\left(\right.$ as $\left.\mathrm{Sr}^{90}-\mathrm{Y}^{90}\right)$ & 0.7 & & \multicolumn{2}{|c|}{$2-6-66$} \\
\hline Gross $\alpha$ as U equivalent $\mu g / 1$ & .8 & & \multicolumn{2}{|c|}{$2-6-66$} \\
\hline \multicolumn{5}{|l|}{ Net extr. $\alpha$, U equivalent $\mu g / I$} \\
\hline \multicolumn{5}{|l|}{ Gross Y (as $\left.\mathrm{Cs}^{137}\right)$} \\
\hline \multicolumn{5}{|l|}{ Potassium } \\
\hline \multicolumn{5}{|l|}{ Strontium $90 *$} \\
\hline \multicolumn{5}{|l|}{ Dissolved solids mg/l } \\
\hline \multicolumn{5}{|l|}{ 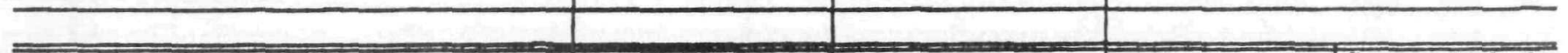 } \\
\hline Isotopic Analysis & & & Method & $\begin{array}{l}\text { Corrected for } \\
\text { decay to: }\end{array}$ \\
\hline \multicolumn{5}{|l|}{ Barium-lanthanum 140} \\
\hline \multicolumn{5}{|l|}{ Cerium-praseodymium ${ }^{146}$} \\
\hline \multicolumn{5}{|l|}{ Cesium ${ }^{137}$} \\
\hline \multicolumn{5}{|l|}{ Chromium ${ }^{7}$} \\
\hline \multicolumn{5}{|l|}{ Cobalt } \\
\hline \multicolumn{5}{|l|}{ Ruthenium_rhodium 106} \\
\hline Tritium (uc/m1 2-1-66) & $\mathrm{ND}$ & & & \\
\hline \multicolumn{5}{|l|}{$\operatorname{Zinc}^{65}$} \\
\hline \multicolumn{5}{|l|}{ Zirconium-niobium 95} \\
\hline & & & & \\
\hline \multicolumn{5}{|l|}{ ND: Sought but not detected by } \\
\hline method used. & & & & \\
\hline
\end{tabular}

Date sample received 12-14-65

Date data released 2-11-66

Program classification

*Analyzed only when indicated by gross $\beta$ count.

Revised form 3-17-65
Analyst T.Curran; P.K.Roscio:H.C.Claassep Date checked

Checked by $2-11 \cdot 66$

$$
\text { V. J. Janzer }
$$

Original Records

Date $2-22-66$ 


\section{U. S. DEPT. OF THE INTERIOR .. GEOLOGICAL SURVEY \\ Statement of Water Analysis}

Source

We11 K28-1

Location

Purvis, Lamar Co., Mississipp1

NW 1/4 NW 1/4_1/4 Soc 28, T 2 2 N, R $15 \mathrm{~W}$. Field/Office No.

Col. By USGS

Field detns: Temp. $\left({ }^{\circ} \mathrm{F}\right)$

Sp. Cond. ( $\mu$ mhos)

Appearance $\mathrm{pH}$

Depth (ft.) 102

Diam. (in.) 2

Water level ( $\mathrm{ft}$.) Unknown

Discharge A few gpm (estimated)

W. B. F. Quaternary (alluvium,

Clear terrace deposits, etc.)

Owner John H. Busha, Rt. 1, Purvis, Miss.

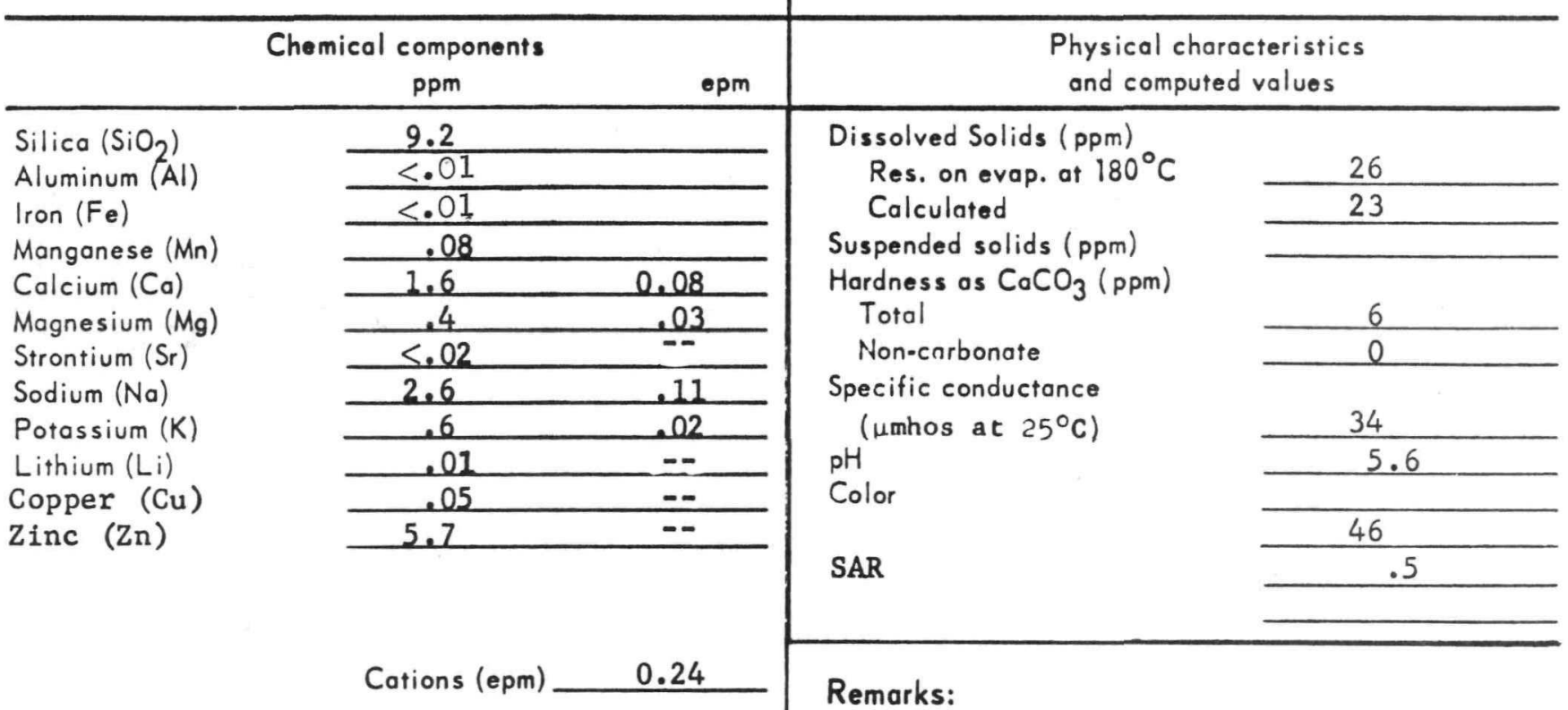

Bicarbonate $\left(\mathrm{HCO}_{3}\right)$

Carbonate $\left(\mathrm{CO}_{3}\right)$

Sulfate $\left(\mathrm{SO}_{4}\right)$

Chloride $(\mathrm{Cl})$

Fluoride ( $F$ )

Nitrate $\left(\mathrm{NO}_{3}\right)$

Phosphate $\left(\mathrm{PO}_{4}\right)$

\begin{tabular}{cc}
10 & 0.16 \\
\hline 0 & .00 \\
\hline$<.1$ & -- \\
\hline 2.4 & .07 \\
\hline$<.1$ & -- \\
\hline .9 & .01 \\
\hline$<.01$ & -- \\
\hline & \\
\hline & \\
\hline
\end{tabular}

Anions (epm)

0.24

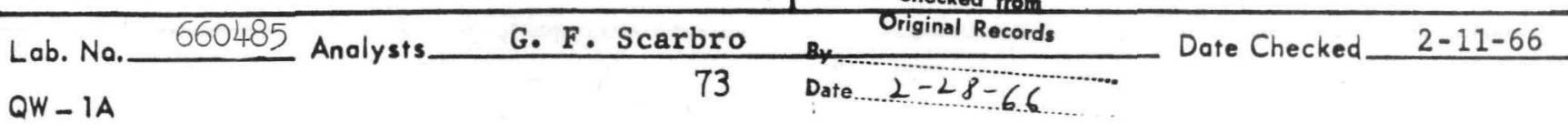


U. S. DEPT. OF THE INTERIOR--GEOLOGICAL SURVEY

Statement of Water Analysis - Radiochemical

Source

Location Purvis, Lamar Co., Mississippi

We11 K28-1

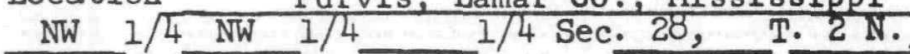

, R. 15 W. Field/Office No.

\section{Date Col. 10-26-65 Time 1520}

Col. By USGS

Field detns: Temp. ( $\left.{ }^{\circ} \mathrm{F}\right) 68$

Sp. Cond. ( $\mu$ mhos)

Appearance

Clear

Well Type Drilled Use Dom; stock

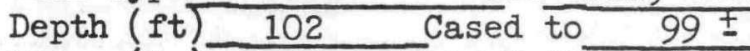

Diam. (in) $\frac{1}{2}$ Date Drilled June 1955

Water level ( $\mathrm{ft}$ ) Unknown

Discharge A few gpm (estimated)

W.B.F. Quaternary (alluvium, terrace deposits, etc.)

Owner_John H. Busha,

Rt. 1, Purvis, Miss.

\begin{tabular}{|c|c|c|c|c|}
\hline Elements & $\mathrm{pc} / \mathrm{l}$ & $\mathrm{pc} / \mathrm{g}$ & \multicolumn{2}{|c|}{ Date determined } \\
\hline \multirow{2}{*}{\multicolumn{5}{|c|}{$\begin{array}{ll}\text { Uranium } & \mu \mathrm{g} / \mathrm{I}\end{array}$}} \\
\hline & & & & \\
\hline Gross B (2.S Sr90 - Y & 0.8 & & \multicolumn{2}{|c|}{$2-6-66$} \\
\hline Gross $\alpha$ as U equivalent $\mu g / 1$ & .8 & & \multicolumn{2}{|c|}{$2-6-66$} \\
\hline \multicolumn{5}{|l|}{ Net extr. $\alpha$, U equivalent $\mu g / 1$} \\
\hline \multicolumn{5}{|l|}{ Gross Y (as $\left.\mathrm{Cs}^{237}\right)$} \\
\hline \multicolumn{5}{|l|}{ Potassium 40} \\
\hline \multicolumn{5}{|l|}{ Strontium $90 *$} \\
\hline \multicolumn{5}{|l|}{ Dissolved solids $\mathrm{mg} / 1$} \\
\hline \multicolumn{5}{|l|}{ 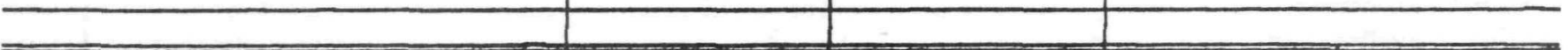 } \\
\hline Isotopic Analysis & & & Method & $\begin{array}{l}\text { Corrected for } \\
\text { decay to: }\end{array}$ \\
\hline \multicolumn{5}{|l|}{ Barium-lanthanum 140} \\
\hline \multicolumn{5}{|l|}{ Cerium-praseodymium ${ }^{146}$} \\
\hline \multicolumn{5}{|l|}{ Cesium ${ }^{137}$} \\
\hline \multicolumn{5}{|l|}{ Chromium 57} \\
\hline \multicolumn{5}{|l|}{ Cobalt } \\
\hline \multicolumn{5}{|l|}{ Ruthenium_rhodium 106} \\
\hline Tritium $\quad(\mu \mathrm{c} / \mathrm{ml} 2-1-66)$ & ND & & & \\
\hline \multicolumn{5}{|l|}{ inc ${ }^{65}$} \\
\hline \multicolumn{5}{|l|}{ Zirconium-niobium ${ }^{95}$} \\
\hline ND: Sought but not detected by & & & & \\
\hline method used. & & & & \\
\hline
\end{tabular}

Date sample received 12-14-65

Date data released $\frac{12-11-66}{2-66}$

Program classification

Analyst P.K.Roscio; H.C.Claassen

Date checked 2-11-66

Checked by V.J. Janzer

*Analyzed only when indlcated by gross $\beta$ count.

Revised form 3-17-65 
U. S. DEPT. OF THE INTERIOR -. GEOLOGICAL SURVEY

Statement of Water Analysis

Source We11 J8-4

Location Lumberton, Lamar Co., Mississippi

SE $1 / 4$ SW $1 / 4 \ldots 1 / 4$ Sec. $8, T .2 N, R_{2} 16$ W. Field/Office No.

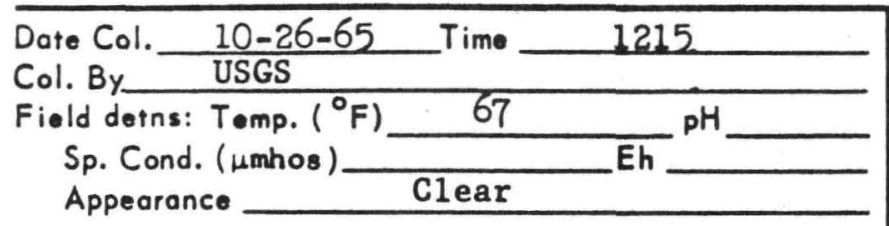

Well Type Drilled

Depth (ft.) 280 Use Bom; stock

Diam. (in.) Date drilled 1959

Water level $(\mathrm{ft})$.70 or 80 below 1.s.d., 1959

Discharge A few gpm (estimated)

W. B. F. Pascagoula and Hattiesburg Fms., undiff.

Owner James R. Boler,

$$
\text { Rt. 4, Lumberton, Miss. }
$$

\section{Chemical components}

Silica $\left(\mathrm{SiO}_{2}\right)$

Aluminum (Al)

Iron ( $\left.F_{e}\right)$

Manganese $(\mathrm{Mn})$

Calcium ( $\mathrm{Ca}$ )

Magnesium (Mg)

Strontium (Sr)

Sodium $(\mathrm{Na})$

Potassium (K)

Lithium ( $L i)$

Copper (Cu)

$\mathrm{Zinc}(\mathrm{Zn})$

Ppm

\begin{tabular}{cc}
41 & \\
\hline$<.01$ & \\
\hline$<.01$ & \\
\hline .28 & \\
\hline 19 & 0.95 \\
\hline 4.7 & .39 \\
\hline .22 & -- \\
\hline 24 & 1.04 \\
\hline 2.4 & .06 \\
\hline .06 & -- \\
\hline .03 & -- \\
\hline .47 & -- \\
\hline
\end{tabular}

Cations (epm)

2.46

Bicarbonate $\left(\mathrm{HCO}_{3}\right)$

Carbonate $\left(\mathrm{CO}_{3}\right)$

Sulfate $\left(\mathrm{SO}_{4}\right)$

Chloride (CI)

Fluoride (F)

Nitrate $\left(\mathrm{NO}_{3}\right)$

Phosphate $\left(\mathrm{PO}_{4}\right)$
Physical characteristics

and computed values

Dissolved Solids (ppm)

Res. on evap. at $180^{\circ} \mathrm{C}$

Calculated

Suspended solids (ppm)

Hardness as $\mathrm{CaCO}_{3}$ (ppm)

Total

Non-carbonate

Specific conductance

(umhos at $25^{\circ} \mathrm{C}$ )

$\mathrm{pH}$

Color

Percent sodium

SAR

$\begin{array}{r}166 \\ \hline 167 \\ \hline \\ 68 \\ \hline 0\end{array}$

$\begin{array}{r}257 \\ \hline 7.6 \\ \hline\end{array}$

$\frac{42}{1.3}$

Remarks:

Anions (epm) $\quad 2.46$

\begin{tabular}{cc}
134 & 2.20 \\
\hline 0 & .00 \\
\hline$<.1$ & - \\
\hline 9.0 & .25 \\
\hline .1 & .01 \\
\hline .2 & .00 \\
\hline$<.01$ & -- \\
\hline & \\
\hline & \\
\hline
\end{tabular}




\section{U. S. DEPT. OF THE INTERIOR .. GEOLOGICAL SURVEY Statement of Water Analysis}

Source We11 K18-1

Location Lumberton, Lamar Co., Mississippi

$\mathrm{NE} 1 / 4 \mathrm{NE} 1 / 4 \ldots 1 / 4 \mathrm{Sec} \cdot 18, \mathrm{~T} \cdot 2 \mathrm{~N} ., \mathrm{R} \cdot 15 \mathrm{~W} \cdot$ Field/Office No.

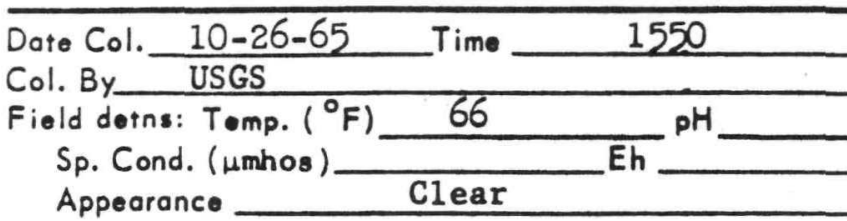

\begin{tabular}{|c|c|c|}
\hline \multicolumn{3}{|c|}{ Chemical components } \\
\hline Silica $\left(\mathrm{SiO}_{2}\right)$ & 8.6 & \\
\hline Aluminum (AI) & .15 & \\
\hline Iron $(\mathrm{Fe})$ & $<.01$ & \\
\hline Manganese $(M n)$ & $<.02$ & \\
\hline Calcium ( $\mathrm{Ca}$ ) & 3.5 & 0.17 \\
\hline Magnesium (Mg) & .5 & .04 \\
\hline Strontium (Sr) & .02 & -- \\
\hline Sodium $(\mathrm{Na})$ & 6.3 & .27 \\
\hline Potassium (K) & .7 & .02 \\
\hline Lithium (Li) & .01 & - \\
\hline Copper (Cu) & .02 & -- \\
\hline Zinc (Zn) & 11 & -- \\
\hline
\end{tabular}

Well Type Bored

Depth (ft.) $56 \pm$

Use Dom

Diam. (in.) 2 Date drilled $1940 \pm$

Water level (ft.) 37.90 below 1.s.d., 8-18-64

Discharge A few gpm (estimated)

W. B. F.

Quaternary (alluvium, terrace deposits, etc.)

Owner W. W. Beech,

Rt. 4, Lumberton, Miss.

\section{Physical characteristics} and computed values

Dissolved Solids (ppm)

Res. on evap. af $180^{\circ} \mathrm{C}$

Calculated

Suspended solids (ppm)

Hardness os $\mathrm{CaCO}_{3}$ (ppm)

Total

Non-carbonate

Specific conductance

( $\mu$ mhos at $25^{\circ} \mathrm{C}$ )

$\mathrm{pH}$

Color

Percent sodium

SAR

$\begin{array}{r}42 \\ \hline 36 \\ \hline 11 \\ \hline 0 \\ \hline\end{array}$

$\begin{array}{r}\frac{52}{6.0} \\ \hline 54 \\ \hline .8 \\ \hline\end{array}$

Cations (epm) $\quad 0.50$

\section{Remarks:}

Bicarbonate $\left(\mathrm{HCO}_{3}\right)$

Carbonate $\left(\mathrm{CO}_{3}\right)$

Sulfate $\left(\mathrm{SO}_{4}\right)$

Chloride $(\mathrm{Cl})$

Fluoride (F)

Nitrate $\left(\mathrm{NO}_{3}\right)$

Phosphate $\left(\mathrm{PO}_{4}\right)$.

\begin{tabular}{cc}
24 & 0.39 \\
\hline 0 & .00 \\
\hline$<.1$ & -- \\
\hline 2.5 & .07 \\
\hline$<.1$ & -- \\
\hline 2.2 & .04 \\
\hline$<.01$ & -- \\
\hline
\end{tabular}

Checked from 
U. S. DEPT. OF THE INTERIOR--GEOLOGICAL SURVEY

Statement of Water Analysis - Radiochemical

Source We11 K18-1

Location Lumberton, Lamar Co., Mississippi
$\mathrm{NE} 1 / 4 \mathrm{NE} 1 / 4$
$1 / 4 \mathrm{Sec} 18, \quad \mathrm{~T} 2 \mathrm{~N}$. , R 15 W. Field/Office No.

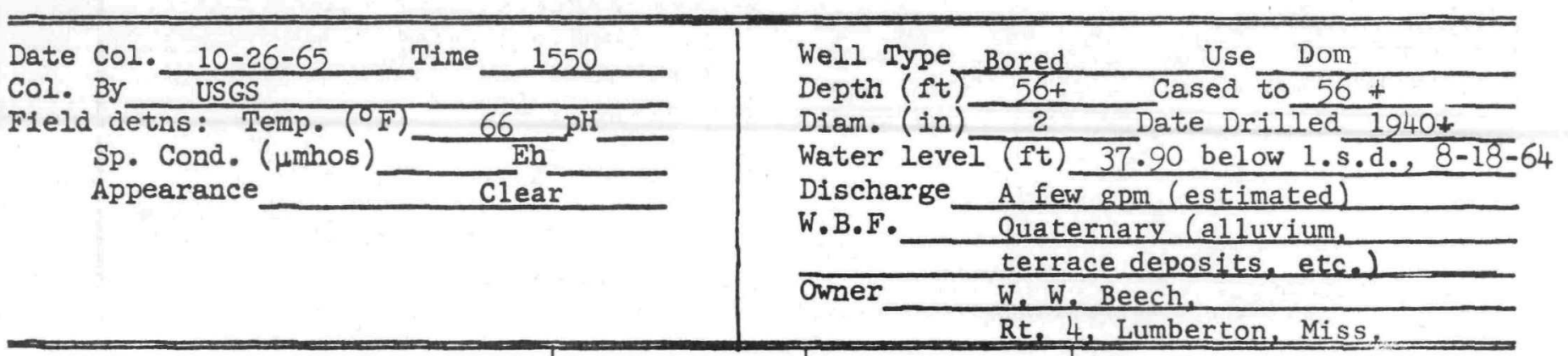

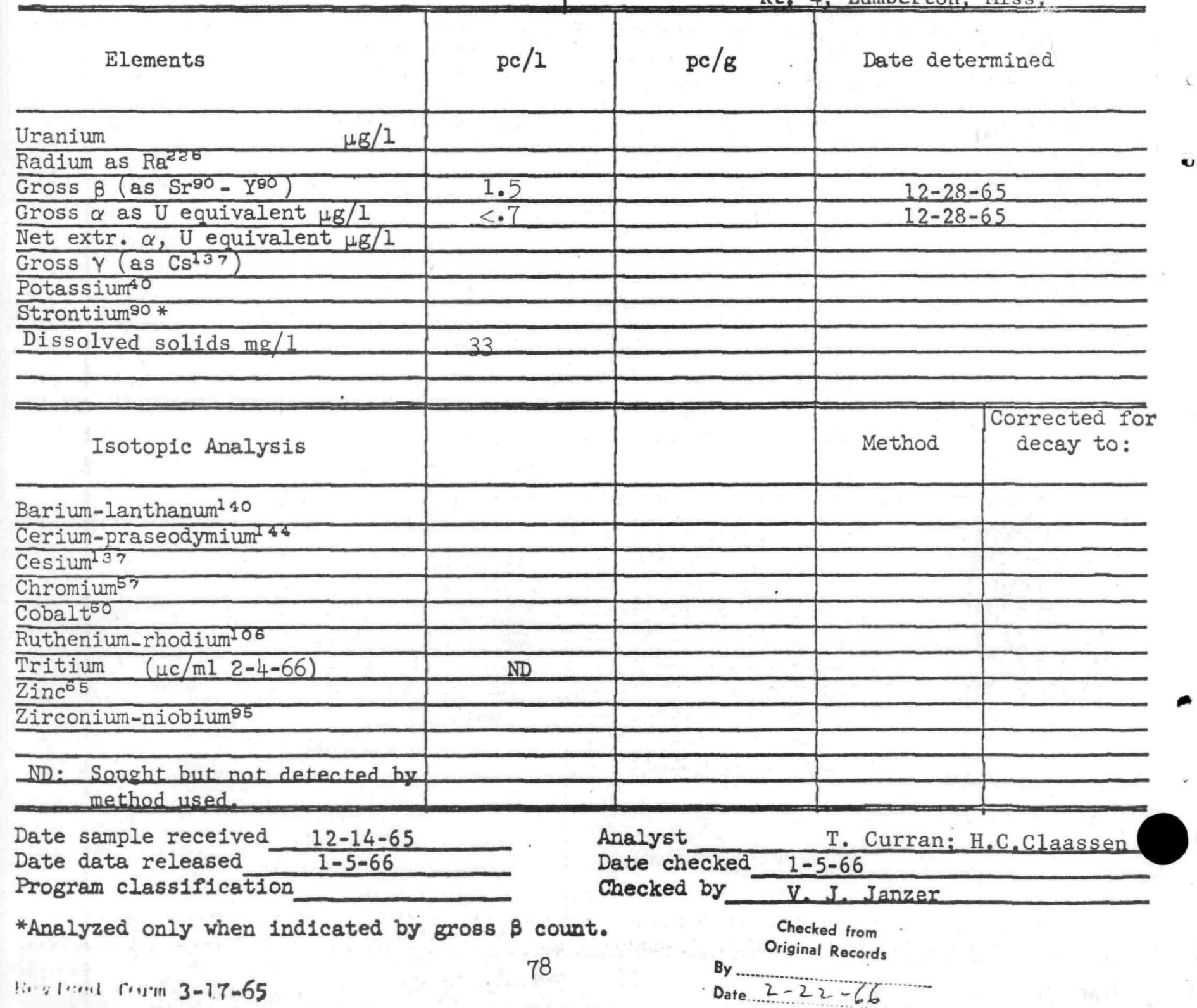




\section{U. S. DEPT. OF THE INTERIOR .. GEOLOGICAL SURVEY Statement of Water Analysis}

Source Water tap at Pure 011 Station north of Lumberton.

Location Lumberton, Lamar Co., Mississippi

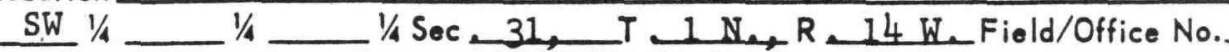

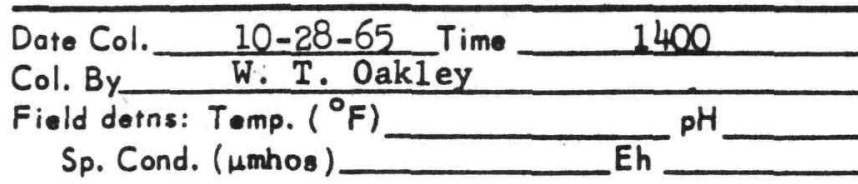

Appearance

Glear

Woll Type Drilled Use Municipal supply

Depth ( $f t$.) See Remarks Cased to See Remarks

Diam. (in.)See Remarks Date drilled See Remarks

Water level (ft.)__ See Remarks

Discharge See Remarks

W. B. F.

See Remarks

Owner Town of Lumberton, Lumberton, Mississippi

\begin{tabular}{|c|c|c|}
\hline \multicolumn{3}{|c|}{ Chemical components } \\
\hline Silica $\left(\mathrm{SiO}_{2}\right)$ & 45 & \\
\hline Aluminum (AI) & $<.01$ & \\
\hline Iron $(\mathrm{Fe})$ & $<.01$ & \\
\hline Manganese $(M n)$ & .03 & \\
\hline Calcium (Ca) & .3 & 0.01 \\
\hline Magnesium (Mg) & .1 & .01 \\
\hline Strontium (Sr) & $<.02$ & $=-$ \\
\hline Sodium $(\mathrm{Na})$ & 49 & 2.13 \\
\hline Potassium (K) & .6 & .02 \\
\hline Lithium (Li) & .02 & $=-$ \\
\hline Copper (Cu) & $<.02$ & - \\
\hline Zinc $(\mathrm{Zn})$ & .08 & $=-$ \\
\hline
\end{tabular}

\section{Physical characteristics} and computed values

Silica $\left(\mathrm{SiO}_{2}\right)$

Aluminum (Al)

$(\mathrm{Fe})$

Manganese (Mn

Calcium (Ca)

Magnesium (Mg)

Strontium $(\mathrm{Sr})$

Sodium ( $\mathrm{Na}$

Potassium (K)

Lithium ( $L i)$

Zinc ( $\mathrm{Zn}$ )

Cations (epm)

2.17

Bicarbonate $\left(\mathrm{HCO}_{3}\right)$

Carbonate $\left(\mathrm{CO}_{3}\right)$

Sulfate $\left(\mathrm{SO}_{4}\right)$

Chloride (CI)

Fluoride (F)

Nitrate $\left(\mathrm{NO}_{3}\right)$

Phosphate $\left(\mathrm{PO}_{4}\right)$

\begin{tabular}{rr}
124 & 2.03 \\
\hline 0 & .00 \\
\hline 7.0 & .15 \\
\hline 3.6 & .10 \\
\hline .1 & .01 \\
\hline .2 & .00 \\
\hline 1.0 & -- \\
\hline
\end{tabular}

Anions (epm) 2.29
Dissolved Solids (ppm)

Res. on evap. af $180^{\circ} \mathrm{C}$

Calculated

164

Suspended solids (ppm)

Hardness as $\mathrm{CaCO}_{3}$ (ppm)

Total

Non-carbonate

Specific conductance

( $\mu$ mhos at $25^{\circ} \mathrm{C}$ )

$\mathrm{pH}$

Color

Percent sodium

SAR

$\begin{array}{r}164 \\ \hline 167 \\ \hline \\ \hline 1 \\ \hline 0\end{array}$

\begin{tabular}{c}
225 \\
\hline 7.3 \\
\hline 98 \\
\hline 21
\end{tabular}

Remarks:

Depth (ft.): No. 1: 1,005; No. 2: 885 .

Cased to: No. 1: $990 \mathrm{ft}$. ; No. 2: --

Diam. (in.): No. 1: 8; No. 2: 10 to 8.

Date drilled: No. 1: 1951; No. 2: 1962.

Water level (ft.): No. 1: 135 below 1.s.d. No. 2: 135 below 1.s.d.

Discharge: No. 1: $700 \mathrm{gpm}$; No. 2: $697 \mathrm{gpm}$.

W.B.F.: No. 1: Catahoula Sandstone (?);

No. 2: Pascagoula and Hattiesburg Fms., undiff. 
U. S. DEPT. OF THE INTERIOR--GEOLOGICAL SURVEY

Statement of Water Analysis - Radiochemical

Source

Water tap at Pure 0il Station north of Lumberton.

Location

Lumberton, Lamar Co., Mississippi

SW $1 / 4$

$1 / 4$

1/4. Sec $31, \quad T$

1 N., R 14 W. Field/Office No.

Date Col. 10-28-65 Time 1400

Col. By W. T. Oakley

Field detns: Temp. (OF)

Sp. Cond. ( $\mu$ mhos)

Appearance
${ }_{\text {Clear }}^{\mathrm{Eh}}$

\section{$\mathrm{pH}$}

\section{(2)}




\section{U. S. DEPT. OF THE INTERIOR .. GEOLOGICAL SURVEY \\ Statement of Water Analysis}

Source Sample collected at tap in County Health Building.

Locotion Purvis, Lamar Co., Mississippi

NW $1 / 4$ NW $1 / 4$ NE 1/4 Sec $16, \quad T, 2 \mathrm{~N}, \mathrm{~T}, 14 \mathrm{~W}$. Field/Office No._L16-2

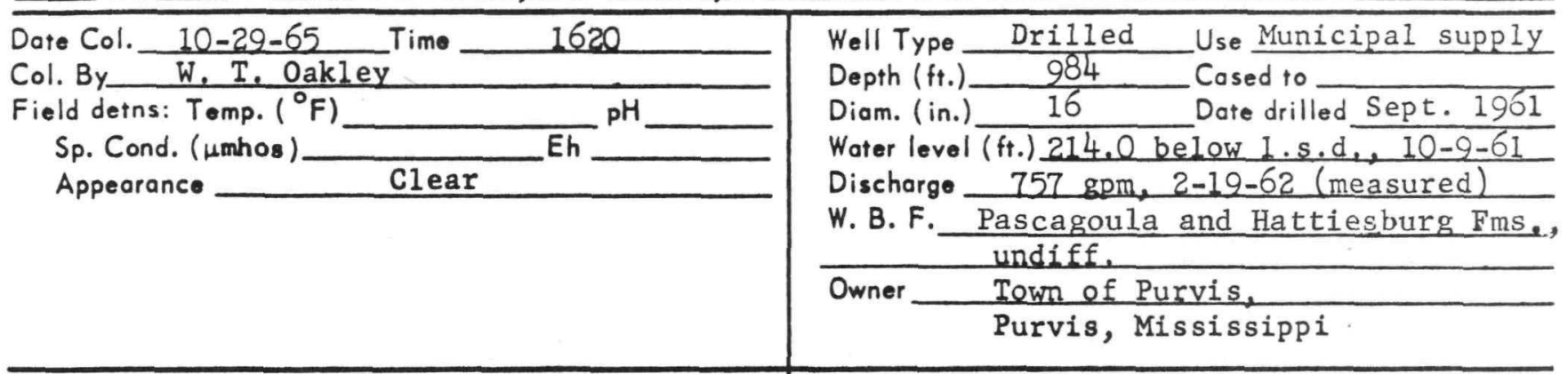

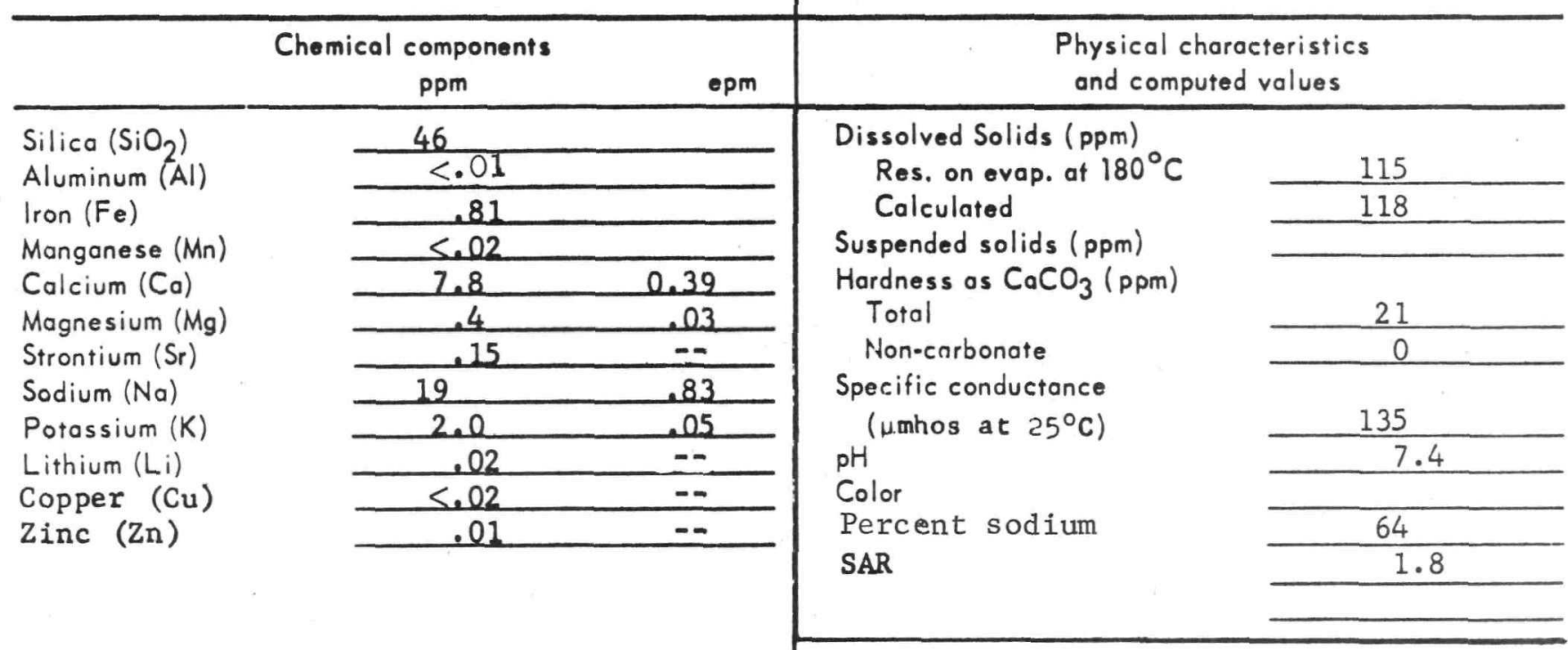

Cations (epm) 1.30 Remarks:

Bicarbonate $\left(\mathrm{HCO}_{3}\right)$

Carbonate $\left(\mathrm{CO}_{3}\right)$

Sulfate $\left(\mathrm{SO}_{4}\right)$

Chloride $(\mathrm{Cl})$

Fluoride ( $F$ )

Nitrate $\left(\mathrm{NO}_{3}\right)$

Phosphate $\left(\mathrm{PO}_{4}\right)$

\begin{tabular}{cc}
62 & 1.02 \\
\hline 0 & .00 \\
\hline 8.0 & .17 \\
\hline 4.3 & .12 \\
\hline$<.1$ & -- \\
\hline .6 & .01 \\
\hline$<.01$ & -- \\
\hline
\end{tabular}

Anions (epm)

1.32 
U. S. DEPT. OF THE INTERIOR--GEOLOGICAL SURVEY

Statement of Water Analysis - Radiochemical

Source Sample collected at tap in County Health Building.

Location Purvis, Lamar Co., Mississippi

$\mathrm{NW} 1 / 4 \mathrm{NW} 1 / 4 \mathrm{NE} 1 / 4 \mathrm{Sec} 16$, T2 N., R 14 W. Field/Office No. L16-2

Date Col. 10-29-65 Time 1620

Col.' By W. T. Oakley

Field detns: Temp. (OF)

$\mathrm{Sp}$. Cond. (Hmhos)

Appearance

clear
Well Type Drilled

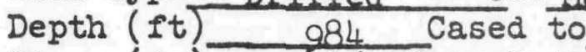

Diam. (In) 16 Date Drilled Sept. 1961

Water level (ft) 214.0 below $1, s, d, 10-9-61$ Discharge $757 \mathrm{gmm}, 2-19-62$ (measured)

W.B.F. Pascagoula and Hattiesburg Fms., undiff.

Owner Town of Purvis,

Purvis, Mississippi

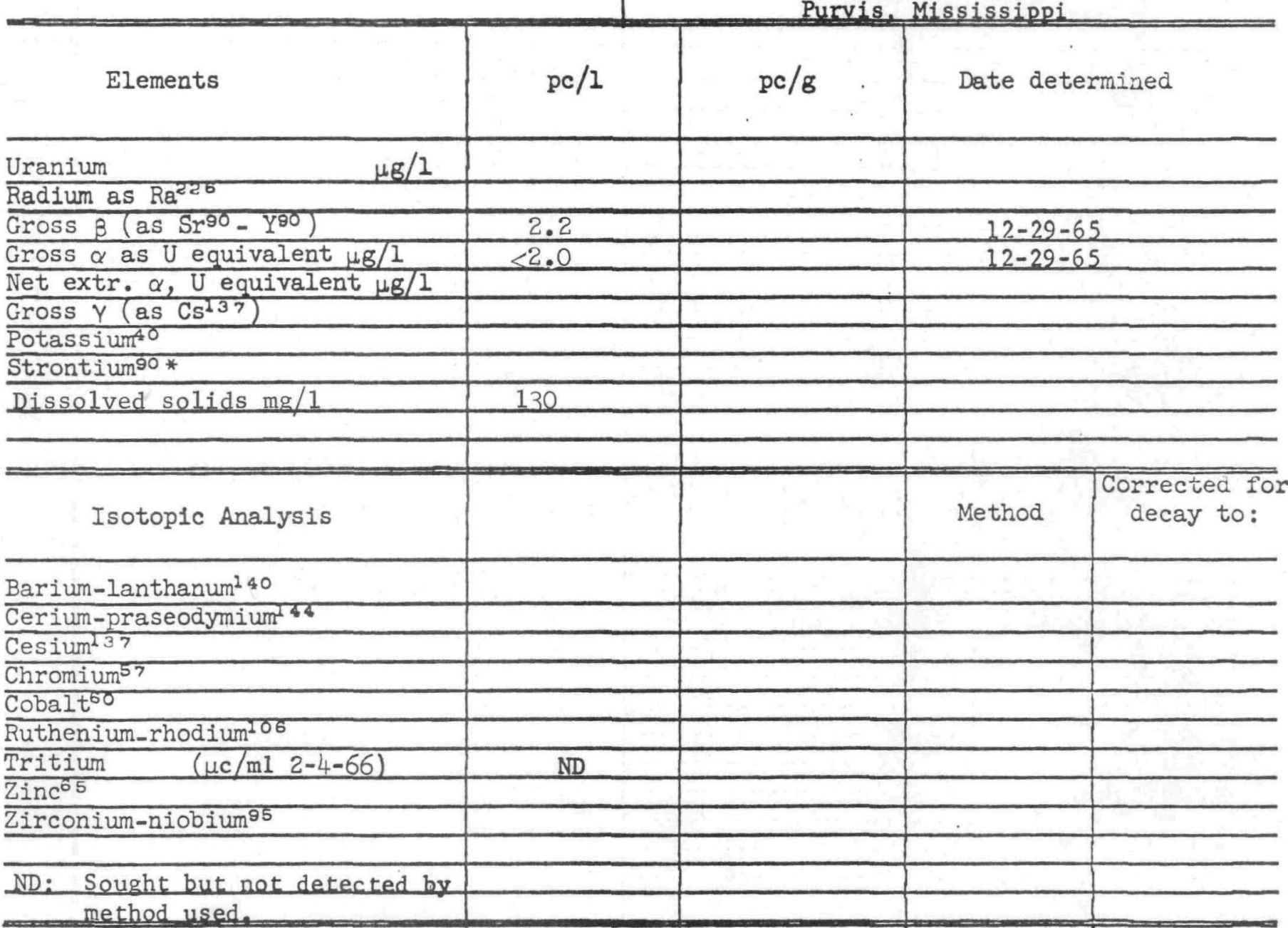

Date sample received 12-14-65

Date data released $1-5-66$

Program classification

Analyst

T. Curran; H.C.Claassen

Date checked 1-5-66

Checked by

*Analyzed only when indicated by gross $\beta$ count.

Revised form 3-17-65
V. J. Janzer

Checked from

Original Records

By

Date..... $2-2=-66$ 


\section{U. S. DEPT. OF THE INTERIOR .. GEOLOGICAL SURVEY \\ Statement of Water Analysis}

Source Sample collected at water tap in AEC Building.

Location Hattiesburg, Forrest Co., Mississippi

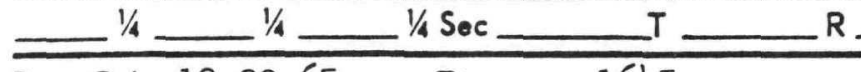

Date Col. 10-29-65_Time 1645

Col. By W. T. Oakley

Field deins: Tomp. $\left({ }^{\circ} \mathrm{F}\right)$

Sp. Cond. (umhos)

Appearance
Field/Office No.

Well Type

Depth (ft.)

Diam. (in.)

Water level (fr.)

Discharge

W. B. F.

Owner

Physical characteristics

and computed values

Dissolved Solids (ppm)

Res. on evap. af $180^{\circ} \mathrm{C}$

Calculated

Suspended solids (ppm)

Hardness as $\mathrm{CaCO}_{3}$ (ppm)

Total

Non-carbonate

Use Municipal supply

Cased to

Dat drilled

$\begin{array}{r}110 \\ \hline 113 \\ \hline \\ \hline 33 \\ \hline 0\end{array}$

Silica $\left(\mathrm{SiO}_{2}\right)$

Aluminum (Al)

Iron ( $\mathrm{Fe})$

Mangane se $(\mathrm{Mn})$

Calcium (Ca)

Mognesium (Mg)

Strontium ( $\mathrm{Sr}$ )

Sodium ( $\mathrm{Na}$ )

Potassium (K)

Lithium ( $\mathrm{Li}$ )

Copper (Cu)

Zinc $(\mathrm{Zn})$

\begin{tabular}{cc}
14 \\
\hline .01 \\
\hline .01 \\
\hline .18 \\
\hline 8.1 & \\
\hline 2.9 & 0.40 \\
\hline .32 & .24 \\
\hline 25 & -- \\
\hline 4.0 & 1.09 \\
\hline .01 & .10 \\
\hline$<.02$ & -- \\
\hline .02 & -- \\
\hline
\end{tabular}

Specific conductance

(umhos at $25^{\circ} \mathrm{C}$ )

$\mathrm{pH}$

Color

Percent sodium

SAR

185

7.7

59

1.9

\section{Cations (epm) $1.83 \quad$ Remarks:}

Bicarbonate $\left(\mathrm{HCO}_{3}\right)$

Carbonate $\left(\mathrm{CO}_{3}\right)$

Sulfate $\left(\mathrm{SO}_{4}\right)$

Chloride $(\mathrm{Cl})$

Fluoride (F)

Nitrate $\left(\mathrm{NO}_{3}\right)$

Phosphate $\left(\mathrm{PO}_{4}\right)$

\begin{tabular}{cc}
96 & 1.57 \\
\hline 0 & .00 \\
\hline 9.0 & .19 \\
\hline 2.3 & .06 \\
\hline .1 & .01 \\
\hline .6 & .01 \\
\hline$<_{.01} 01$ & -- \\
\hline
\end{tabular}

Water supply comes from several wells in two separate fields.
Checked from

Original Records

Lab. No. 660490 Analysts G. F. Scarbro

$Q W-1 A$

Anions (epm) $\quad 1.84$ Date Checked 2-11-66 
U. S. DEPT. OF THE INTERIOR--GEOLOGICAL SURVEY

Statement of Water Analysis - Radiochemical

Source Sample collected at water tap in AEC Building.

Location Hattiesburg, Forrest Co.. Mississippi
$1 / 4$
$1 / 4$
$1 / 4 \mathrm{Sec}$
$\mathrm{R}$
Field/Office No.

Date Col. 10-29-65 Time 1645 Well Type

Col. By W. T. Oakley

Field detns: Temp. (OF)

Sp. Cond. ( $\mu$ mhos)

Appearance
Depth ( $\mathrm{et}$ )

Diam. (In)

Water level (ft)

Discharge

W.B.F.
Use Municipal supply

Cased to

Date Drilled

\section{Owner}

\begin{tabular}{|c|c|c|}
\hline Elements & $\mathrm{pc} / \mathrm{l}$ & Date determined \\
\hline Uranium $\mu \mathrm{g} / 1$ & & $\therefore$ \\
\hline \multicolumn{3}{|l|}{ Radium as $\mathrm{Ra}^{226}$} \\
\hline Gross $\beta\left(\right.$ as $\left.\mathrm{Sr}^{90}-\mathrm{Y}^{80}\right)$ & 3.8 & $12-29-65$ \\
\hline Gross $\alpha$ as $\mathrm{U}$ equivalent $\mu \mathrm{g} / \mathrm{I}$ & 3.0 & $12-29-65$ \\
\hline Net extr. $\alpha$, U equivalent $\mu g / 1$ & & \\
\hline Gross $Y\left(\operatorname{as~} \mathrm{Cs}^{237}\right)$ & & \\
\hline \multicolumn{3}{|l|}{ Potassium } \\
\hline \multicolumn{3}{|l|}{ Strontium 90 * } \\
\hline Dissolved solids mg/l & 120 & \\
\hline \multicolumn{3}{|l|}{ 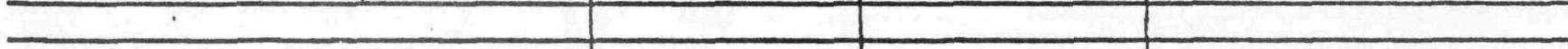 } \\
\hline \multicolumn{2}{|l|}{ Isotopic Analysis } & \multirow{4}{*}{$\begin{array}{l}\text { Remarks: } \\
\text { Water supply comes from several } \\
\text { wells in two separate fields. }\end{array}$} \\
\hline & \\
\hline \multicolumn{2}{|l|}{ Cerium-praseodymium ${ }^{46}$} & \\
\hline \multicolumn{2}{|l|}{ Cesium ${ }^{37} 7$} & \\
\hline \multicolumn{2}{|l|}{$\begin{array}{l}\text { Chromium } \\
\text { Cobalt }\end{array}$} & \multirow{2}{*}{$\begin{array}{l}\text { ND: Sought but not detected by method } \\
\text { used } 2-4-66 \text {. }\end{array}$} \\
\hline Cobalt & - & \\
\hline \multicolumn{2}{|l|}{ Ruthenium-rhodium 106} & \\
\hline Tritium & $\mathrm{ND}$ & \\
\hline \multicolumn{2}{|l|}{ inc ${ }^{65}$} & . \\
\hline \multicolumn{2}{|l|}{ Zirconium-niobium $^{96}$} & \\
\hline 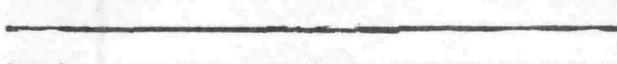 & & \\
\hline & & \\
\hline
\end{tabular}

Date sample received 12-14-65

Date data released 1-5-66

Program classification

*Analyzed only when indicated by gross $\beta$ count.

Revised form 3-17-65
Analyst

T. Curran; H.C.Claassen

Date checked 1-5-66

Checked by

Original Records 
US GS $-474-120$

Dribble -53

\section{Distribution:}

Nevada Operations Office, U.S. Atomic Energy Commission, Las Vegas, Nevada:

D. M. Hame1, NVOO Technical Library

Technical Information Center, U.S. Atomic Energy Commission, Oak Ridge, Tennessee:

(2) 